\title{
Copper-catalyzed Aldol Reaction of Vinyl Azides with Trifluoromethyl Ketones
}

Zhenhua Liu, ${ }^{\dagger}$ Zhihai Zhang, ${ }^{\dagger}$ Guangyu Zhu, ${ }^{\dagger}$ Yiqin Zhou, ${ }^{\dagger}$ Lin Yang,,${ }^{\dagger}$ Wen Gao*, ${ }^{\dagger}$ Lili Tong $^{\dagger}$ and Bo Tang* ${ }^{*} \dagger$

${ }^{\dagger}$ College of Chemistry, Chemical Engineering and Materials Science, Collaborative Innovation Center of Functionalized Probes for Chemical Imaging in Universities of Shandong, Key Laboratory of Molecular and Nano Probes, Ministry of Education, Shandong Provincial Key Laboratory of Clean Production of Fine Chemicals, Shandong Normal University, Jinan, 250014, P. R. China

E-mail: gaowen@sdnu.edu.cn; tangb@sdnu.edu.cn

\section{Contents}

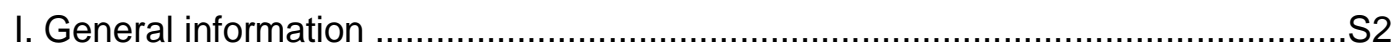

II. Synthesis and analytical data of compounds $\mathbf{3}$ and 4 ............................S2-S15

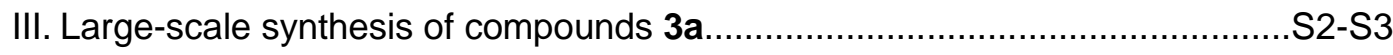

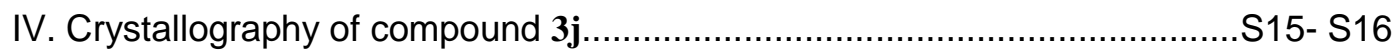

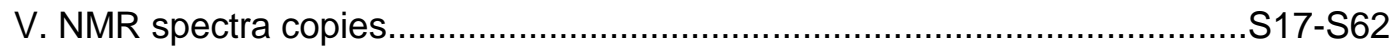




\section{General information}

All reagents were purchased from commercial sources and used without treatment, unless otherwise indicated. The products were purified by column chromatography over silica gel. ${ }^{1} \mathrm{HNMR}$ and ${ }^{13} \mathrm{C}$ NMR spectra were recorded at $25^{\circ} \mathrm{C}$ on a Varian $400 \mathrm{MHz}$ and $101 \mathrm{MHz}$, respectively, and TMS was used as internal standard. Mass spectra were recorded on BRUKER AutoflexIII Smartbeam MS-spectrometer. High resolution mass spectra (HRMS) were recorded on Bruck microTof by using ESI method.

\section{Synthesis and analytical data of compounds 3 and 4.}<smiles>C=C(N)c1ccccc1</smiles>

$1 \mathrm{a}$<smiles>O=C(c1ccccc1)C(F)(F)F</smiles>

$2 a$

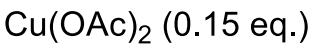

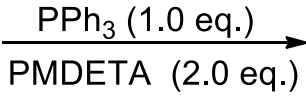
DMSO, $40^{\circ} \mathrm{C}$<smiles>O=C(CC(O)(c1ccccc1)C(F)(F)F)c1ccccc1</smiles>

$3 a$

Typical synthetic procedure (with $\mathbf{3 a}$ as an example): To a solution of (1-azidovinyl)benzene (1a) (72 mg, $0.5 \mathrm{mmol})$, trifluoroacetophenone (2a) $(0.104 \mathrm{~mL}$, $0.6 \mathrm{mmol})$, pentamethyldiethylenetriamine (PMDETA) $(0.208 \mathrm{~mL}, 1.0 \mathrm{mmol})$ and $\mathrm{PPh}_{3}(0.13 \mathrm{~g}, 0.5 \mathrm{mmol})$ in DMSO $(1 \mathrm{~mL})$ at $40{ }^{\circ} \mathrm{C}, \mathrm{CuI}(14 \mathrm{mg}, 0.075 \mathrm{mmol})$ was added. The reaction mixture was then stirred for $8 \mathrm{~h}$ when TLC conformed that substrate 1a had been consumed. The resulting reaction mixture was cooled to room temperature and taken up by dichloromethane $(3 \times 15 \mathrm{~mL})$. The organic layer was washed with brine $(3 \times 40 \mathrm{~mL})$, dried over $\mathrm{MgSO}_{4}$ and concentrated. Purification of the crude product via flash column chromatography (silica gel; petroleum ether/ethyl acetate $(60: 1)$ and concentratinon in vacuo afforded $\mathbf{3 a}(0.13 \mathrm{~g})$ in $90 \%$ yield.

\section{Large-scale synthesis of compounds 3a} $1 \mathrm{mmol}-\mathrm{scale}$ synthesis of compounds $3 \mathrm{a}$

To a solution of (1-azidovinyl)benzene (1a) $(0.15 \mathrm{~g}, 1 \mathrm{mmol})$, trifluoroacetophenone (2a) $(0.208 \mathrm{~mL}, 1.2 \mathrm{mmol})$, pentamethyldiethylenetriamine (PMDETA) (0.416 mL, 
$2.0 \mathrm{mmol})$ and $\mathrm{PPh}_{3}(0.26 \mathrm{~g}, 1 \mathrm{mmol}) \quad$ in DMSO $(2 \mathrm{~mL})$ at $40{ }^{\circ} \mathrm{C}, \mathrm{CuI}(28 \mathrm{mg}, 0.15$ mmol) was added. The reaction mixture was then stirred for $8 \mathrm{~h}$ when TLC conformed that substrate 1a had been consumed. The resulting reaction mixture was cooled to room temperature and taken up by dichloromethane $(3 \times 15 \mathrm{~mL})$. The organic layer was washed with brine $(3 \times 40 \mathrm{~mL})$, dried over $\mathrm{MgSO}_{4}$ and concentrated. Purification of the crude product via flash column chromatography (silica gel; petroleum ether/ethyl acetate $(60: 1)$ and concentratinon in vacuo afforded $\mathbf{3 a}(0.26 \mathrm{~g})$ in $88 \%$ yield.

\section{$5 \mathrm{mmol}-\mathrm{scale}$ synthesis of compounds $3 \mathrm{a}$}

To a solution of (1-azidovinyl)benzene (1a) $(0.72 \mathrm{~g}, 5 \mathrm{mmol})$, trifluoroacetophenone (2a) $(1.04 \mathrm{~mL}, 6 \mathrm{mmol})$, pentamethyldiethylenetriamine (PMDETA) (2.08 $\mathrm{mL}, 10$ $\mathrm{mmol})$ and $\mathrm{PPh}_{3}(1.30 \mathrm{~g}, 5 \mathrm{mmol})$ in DMSO $(10 \mathrm{~mL})$ at $40{ }^{\circ} \mathrm{C}, \mathrm{CuI}(0.14 \mathrm{~g}, 0.75$ mmol) was added. The reaction mixture was then stirred for $8 \mathrm{~h}$ when TLC conformed that substrate 1a had been consumed. The resulting reaction mixture was cooled to room temperature and taken up by dichloromethane $(3 \times 20 \mathrm{~mL})$. The organic layer was washed with brine $(3 \times 60 \mathrm{~mL})$, dried over $\mathrm{MgSO}_{4}$ and concentrated. Purification of the crude product via flash column chromatography (silica gel; petroleum ether/ethyl acetate $(60: 1)$ and concentratinon in vacuo afforded $\mathbf{3 a}(1.25 \mathrm{~g})$ in $85 \%$ yield.<smiles>O=C(CC(O)(c1ccccc1)C(F)(F)F)c1ccccc1</smiles>

(3a) Purification via flash column chromatography (silica gel; petroleum ether/ethyl acetate (60 : 1); Colourless liquid; $0.13 \mathrm{~g} ;{ }^{1} \mathbf{H}$ NMR (400 MHz, $\left.\mathrm{CDCl}_{3}\right) \delta$ 7.99-7.90 (m, 2H), 7.68-7.59 (m, 3H), 7.55-7.43 (m, 2H), 7.40-7.31 (m, 3H), 5.69 (s, 1H), 4.05 $(\mathrm{d}, J=17.4 \mathrm{~Hz}, 1 \mathrm{H}), 3.66(\mathrm{~d}, J=17.4 \mathrm{~Hz}, 1 \mathrm{H}) ;{ }^{13} \mathrm{C} \mathbf{N M R}\left(100 \mathrm{MHz}, \mathrm{CDCl}_{3}\right) \delta$ $199.71,137.69,136.32,134.46,128.96,128.76,128.46,128.25,126.32,124.62$ (q, $J$ $=282.9 \mathrm{~Hz}), 76.39$ (q, $J=28.9 \mathrm{~Hz}), 40.24 ;{ }^{19} \mathbf{F}$ NMR $\left(376 \mathrm{MHz}, \mathrm{CDCl}_{3}\right) \delta-80.20$; HRMS (ESI) $\mathrm{m} / \mathrm{z}$ calculated for $\mathrm{C}_{16} \mathrm{H}_{13} \mathrm{~F}_{3} \mathrm{O}_{2}[\mathrm{M}+\mathrm{H}]^{+}$: 295.0946, found: 295.0939 . 


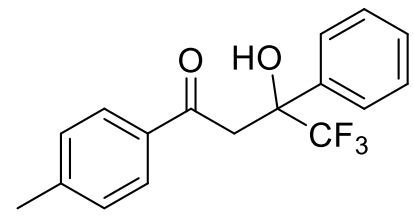

(3b) Purification via flash column chromatography (silica gel; petroleum ether/ethyl acetate (60 : 1); White solid; $0.15 \mathrm{~g} ;{ }^{1} \mathbf{H}$ NMR (400 MHz, $\left.\mathrm{CDCl}_{3}\right) \delta$ 7.87-7.79 (m, 2H), 7.65-7.56 (m, 2H), 7.40-7.26 (m, 5H), $5.80(\mathrm{~s}, 1 \mathrm{H}), 4.00(\mathrm{~d}, J=17.2 \mathrm{~Hz}, 1 \mathrm{H})$, $3.60(\mathrm{~d}, J=17.2 \mathrm{~Hz}, 1 \mathrm{H}), 2.42(\mathrm{~s}, 3 \mathrm{H}) ;{ }^{13} \mathrm{C} \mathbf{N M R}\left(100 \mathrm{MHz}, \mathrm{CDCl}_{3}\right) \delta$ 199.30, 145.67, 137.79, 133.88, 129.64, 128.70, 128.41, 126.33, 124.64 (q, $J=283.0 \mathrm{~Hz})$, $76.56(\mathrm{q}, J=28.9 \mathrm{~Hz}), 39.93,21.78 ;{ }^{19} \mathbf{F}$ NMR $\left(376 \mathrm{MHz}, \mathrm{CDCl}_{3}\right) \delta-80.18$; HRMS (ESI) $\mathrm{m} / \mathrm{z}$ calculated for $\mathrm{C}_{17} \mathrm{H}_{15} \mathrm{~F}_{3} \mathrm{O}_{2}[\mathrm{M}+\mathrm{H}]^{+}: 309.1102$, found: 309.1098 .

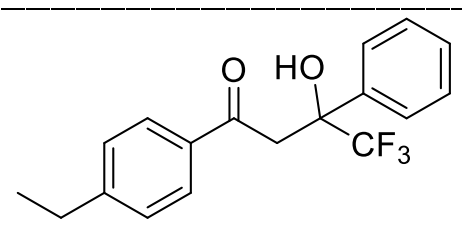

(3c) Purification via flash column chromatography (silica gel; petroleum ether/ethyl acetate (60 : 1); Colourless liquid; $0.15 \mathrm{~g} ;{ }^{1} \mathbf{H}$ NMR (400 MHz, $\left.\mathrm{CDCl}_{3}\right) \delta$ 7.89-7.83 (m, 2H), 7.64-7.57 (m, 2H), 7.39-7.28 (m, 5H), $5.81(\mathrm{~s}, 1 \mathrm{H}), 4.01(\mathrm{~d}, J=17.3 \mathrm{~Hz}$, $1 \mathrm{H}), 3.61(\mathrm{~d}, J=17.3 \mathrm{~Hz}, 1 \mathrm{H}), 2.77-2.66(\mathrm{~m}, 2 \mathrm{H}), 1.26(\mathrm{t}, J=7.6 \mathrm{~Hz}, 3 \mathrm{H}) ;{ }^{13} \mathbf{C} \mathbf{N M R}$ $\left(100 \mathrm{MHz}, \mathrm{CDCl}_{3}\right) \delta 199.31,151.76,137.79,134.07,128.69,128.51,128.45,128.41$, 126.33, $124.63(\mathrm{q}, J=282.9 \mathrm{~Hz}), 76.25(\mathrm{q}, J=28.9 \mathrm{~Hz}), 39.96,29.03,15.06 ;{ }^{19} \mathbf{F}$ NMR (376 MHz, $\left.\mathrm{CDCl}_{3}\right) \delta-80.21$; HRMS (ESI) m/z calculated for $\mathrm{C}_{18} \mathrm{H}_{17} \mathrm{~F}_{3} \mathrm{O}_{2}$ $[\mathrm{M}+\mathrm{H}]^{+}:$323.1259, found: 323.1246 .

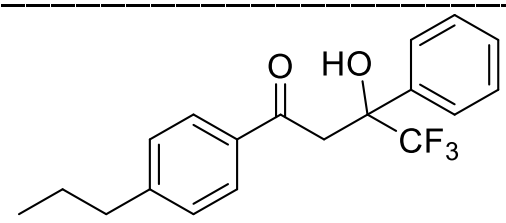

(3d) Purification via flash column chromatography (silica gel; petroleum ether/ethyl acetate (60 : 1); Colourless liquid; $0.14 \mathrm{~g} ;{ }^{1} \mathbf{H}$ NMR (400 $\left.\mathrm{MHz}, \mathrm{CDCl}_{3}\right) \delta 7.82-7.75$ (m, 2H), $7.54(\mathrm{~d}, J=7.4 \mathrm{~Hz}, 2 \mathrm{H}), 7.33-7.19(\mathrm{~m}, 5 \mathrm{H}), 5.73(\mathrm{~s}, 1 \mathrm{H}), 3.94(\mathrm{~d}, J=17.3$ $\mathrm{Hz}, 1 \mathrm{H}), 3.54$ (d, $J=17.3 \mathrm{~Hz}, 1 \mathrm{H}), 2.63-2.54(\mathrm{~m}, 2 \mathrm{H}), 1.67-1.54(\mathrm{~m}, 2 \mathrm{H}), 0.93-0.83$ $(\mathrm{m}, 3 \mathrm{H}) ;{ }^{13} \mathrm{C}$ NMR $\left(100 \mathrm{MHz}, \mathrm{CDCl}_{3}\right) \delta 199.31,150.31,137.76,134.04,129.05$, $128.70,128.42,126.33,124.61(\mathrm{q}, J=282.9 \mathrm{~Hz}), 76.52$ (q, $J=29.1 \mathrm{~Hz}), 39.95,38.10$, 
24.16, 13.77; ${ }^{19}$ F NMR $\left(376 \mathrm{MHz}, \mathrm{CDCl}_{3}\right) \delta$-80.24; HRMS (ESI) m/z calculated for $\mathrm{C}_{19} \mathrm{H}_{19} \mathrm{~F}_{3} \mathrm{O}_{2}[\mathrm{M}+\mathrm{H}]^{+}:$337.1415, found: 337.1405.

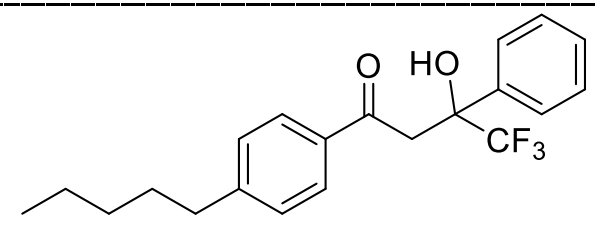

(3e) Purification via flash column chromatography (silica gel; petroleum ether/ethyl acetate (60 : 1); Colourless liquid; $0.15 \mathrm{~g} ;{ }^{1} \mathbf{H}$ NMR (400 $\left.\mathrm{MHz}, \mathrm{CDCl}_{3}\right) \delta 7.89-7.82$ $(\mathrm{m}, 2 \mathrm{H}), 7.65-7.57(\mathrm{~m}, 2 \mathrm{H}), 7.39-7.27(\mathrm{~m}, 5 \mathrm{H}), 5.80(\mathrm{~s}, 1 \mathrm{H}), 4.02(\mathrm{~d}, J=17.3 \mathrm{~Hz}$ $1 \mathrm{H}), 3.61(\mathrm{~d}, J=17.3 \mathrm{~Hz}, 1 \mathrm{H}), 2.72-2.62(\mathrm{~m}, 2 \mathrm{H}), 1.70-1.59(\mathrm{~m}, 2 \mathrm{H}), 1.40-1.27$ (m, 4H), 0.95-0.83 (m, 3H); ${ }^{13} \mathbf{C}$ NMR (100 MHz, $\left.\mathrm{CDCl}_{3}\right) \delta 199.30,150.59,137.76$, $134.00,128.99,128.70,128.43,126.33,124.61$ (q, $J=282.9 \mathrm{~Hz}), 76.52$ (q, $J=28.9$ $\mathrm{Hz}), 39.95,36.06,31.42,30.71,22.49,14.01 ;{ }^{19} \mathbf{F}$ NMR $\left(376 \mathrm{MHz}, \mathrm{CDCl}_{3}\right) \delta-80.24$; HRMS (ESI) $\mathrm{m} / \mathrm{z}$ calculated for $\mathrm{C}_{21} \mathrm{H}_{23} \mathrm{~F}_{3} \mathrm{O}_{2}[\mathrm{M}+\mathrm{H}]^{+}:$: 365.1728, found: 365.1732 .

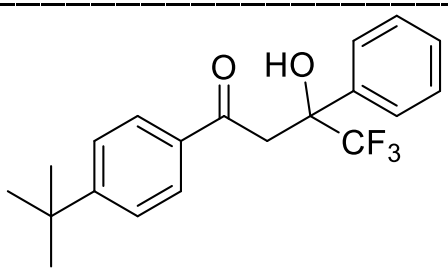

(3f) Purification via flash column chromatography (silica gel; petroleum ether/ethyl acetate (60 : 1); White solid; $0.15 \mathrm{~g} ;{ }^{1} \mathbf{H}$ NMR (400 MHz, $\left.\mathrm{CDCl}_{3}\right) \delta$ 7.90-7.85 (m, 2H), 7.64-7.58 (m, 2H), 7.53-7.47 (m, 2H), 7.39-7.30 (m, 3H), $5.81(\mathrm{~s}, 1 \mathrm{H}), 4.01(\mathrm{~d}$, $J=17.3 \mathrm{~Hz}, 1 \mathrm{H}), 3.62(\mathrm{~d}, J=17.3 \mathrm{~Hz}, 1 \mathrm{H}), 1.34(\mathrm{~s}, 9 \mathrm{H}) ;{ }^{13} \mathbf{C}$ NMR $(100 \mathrm{MHz}$, $\left.\mathrm{CDCl}_{3}\right) \delta 199.31,158.53,137.78,133.74,128.70,128.42,128.27,126.33,125.93$, $124.62(\mathrm{q}, J=282.8 \mathrm{~Hz}), 76.53(\mathrm{q}, J=29.1 \mathrm{~Hz}), 39.96,35.31,31.01 ;{ }^{19}$ F NMR $(376$ $\mathrm{MHz}, \mathrm{CDCl}_{3}$ ) $\delta$-80.22; HRMS (ESI) $\mathrm{m} / \mathrm{z}$ calculated for $\mathrm{C}_{20} \mathrm{H}_{21} \mathrm{~F}_{3} \mathrm{O}_{2}[\mathrm{M}+\mathrm{H}]^{+}$: 351.1572, found: 351.1578 .<smiles>COc1ccc(C(=O)CC(O)(c2ccccc2)C(F)(F)F)cc1</smiles> 
(3g) Purification via flash column chromatography (silica gel; petroleum ether/ethyl acetate (60 : 1); Colourless liquid; $0.16 \mathrm{~g} ;{ }^{1} \mathbf{H}$ NMR (400 MHz, $\mathrm{CDCl}_{3}$ ) $\delta 7.97-7.86$ (m, 2H), 7.65-7.56 (m, 2H), 7.40-7.29 (m, 3H), 6.99-6.89 (m, 2H), $5.92(\mathrm{~s}, 1 \mathrm{H}), 3.96$ $(\mathrm{d}, J=17.1 \mathrm{~Hz}, 1 \mathrm{H}), 3.88(\mathrm{~s}, 3 \mathrm{H}), 3.57(\mathrm{~d}, J=17.1 \mathrm{~Hz}, 1 \mathrm{H}) ;{ }^{13} \mathbf{C}$ NMR $(100 \mathrm{MHz}$, $\left.\mathrm{CDCl}_{3}\right) \delta 198.04,164.63,137.86,130.76,129.35,128.67,128.40,126.34,124.66(\mathrm{q}$, $J=283.1 \mathrm{~Hz}), 114.13,76.87(\mathrm{q}, J=31.8 \mathrm{~Hz}), 55.63,39.54 ;{ }^{19} \mathbf{F}$ NMR $(376 \mathrm{MHz}$, $\left.\mathrm{CDCl}_{3}\right) \delta$-80.19; HRMS (ESI) $\mathrm{m} / \mathrm{z}$ calculated for $\mathrm{C}_{17} \mathrm{H}_{15} \mathrm{~F}_{3} \mathrm{O}_{3}[\mathrm{M}+\mathrm{H}]^{+}:$: 325.1052, found: 325.1046 .

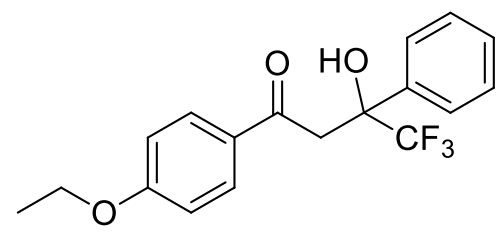

(3h) Purification via flash column chromatography (silica gel; petroleum ether/ethyl acetate (60 : 1); Colourless liquid; 0.16 g; ${ }^{1} \mathbf{H}$ NMR (400 MHz, $\left.\mathrm{CDCl}_{3}\right) \delta 7.99-7.86$ (m, 2H), $7.61(\mathrm{~d}, J=7.2 \mathrm{~Hz}, 2 \mathrm{H}), 7.45-7.29(\mathrm{~m}, 3 \mathrm{H}), 7.00-6.86(\mathrm{~m}, 2 \mathrm{H}), 5.95(\mathrm{~s}, 1 \mathrm{H})$, 4.16-4.05 (m, 2H), $3.96(\mathrm{~d}, J=17.1 \mathrm{~Hz}, 1 \mathrm{H}), 3.56(\mathrm{~d}, J=17.1 \mathrm{~Hz}, 1 \mathrm{H}), 1.49-1.38(\mathrm{~m}$, 3H); ${ }^{13}$ C NMR (100 MHz, $\left.\mathrm{CDCl}_{3}\right) \delta 198.01,164.07,137.87,130.76,129.13,128.64$, 128.37, 126.33, 124.65 (q, $J=283.0 \mathrm{~Hz}), 114.52,76.55$ (q, $J=28.7 \mathrm{~Hz}$ ), 64.00, 39.49, 14.61; ${ }^{19} \mathbf{F}$ NMR $\left(376 \mathrm{MHz}, \mathrm{CDCl}_{3}\right) \delta-80.20$; HRMS (ESI) m/z calculated for $\mathrm{C}_{18} \mathrm{H}_{17} \mathrm{~F}_{3} \mathrm{O}_{3}[\mathrm{M}+\mathrm{H}]^{+}: 339.1208$, found: 339.1200 .<smiles>O=C(CC(O)(c1ccccc1)C(F)(F)F)c1ccc(F)cc1</smiles>

(3i) Purification via flash column chromatography (silica gel; petroleum ether/ethyl acetate (60 : 1); Colourless liquid; $0.14 \mathrm{~g} ;{ }^{1} \mathbf{H}$ NMR (400 MHz, $\left.\mathrm{CDCl}_{3}\right) \delta 8.03-7.93$ (m, 2H), 7.65-7.55 (m, 2H), 7.41-7.31 (m, 3H), 7.21-7.11 (m, 2H), $5.60(\mathrm{~s}, 1 \mathrm{H}), 4.00$ $(\mathrm{d}, J=17.3 \mathrm{~Hz}, 1 \mathrm{H}), 3.62(\mathrm{~d}, J=17.2 \mathrm{~Hz}, 1 \mathrm{H}) ;{ }^{13} \mathrm{C} \mathbf{N M R}\left(100 \mathrm{MHz}, \mathrm{CDCl}_{3}\right) \delta$ $197.95,166.53$ (d, $J=256.0 \mathrm{~Hz}), 137.54,132.71(\mathrm{q}, J=2.9 \mathrm{~Hz}), 131.67$ (d, $J=9.6$ $\mathrm{Hz}), 128.82,128.49,126.25,124.54(\mathrm{q}, J=282.9 \mathrm{~Hz}), 116.21(\mathrm{~d}, J=22.0 \mathrm{~Hz}), 76.48$ 
$(\mathrm{d}, J=29.0 \mathrm{~Hz}), 40.19 ;{ }^{19} \mathbf{F}$ NMR $\left(376 \mathrm{MHz}, \mathrm{CDCl}_{3}\right) \delta-80.22,-102.34 ;$ HRMS (ESI) $\mathrm{m} / \mathrm{z}$ calculated for $\mathrm{C}_{16} \mathrm{H}_{12} \mathrm{~F}_{4} \mathrm{O}_{2}[\mathrm{M}+\mathrm{H}]^{+}: 313.0852$, found: 313.0833 .<smiles>O=C(CC(O)(c1ccccc1)C(F)(F)F)c1ccc(Br)cc1</smiles>

(3j) Purification via flash column chromatography (silica gel; petroleum ether/ethyl acetate (60 : 1); White solid; $0.17 \mathrm{~g} ;{ }^{1} \mathbf{H}$ NMR (400 MHz, $\left.\mathrm{CDCl}_{3}\right) \delta$ 7.83-7.73 (m, 2H), 7.66-7.60 (m, 2H), 7.62-7.55 (m, 2H), 7.40-7.29 (m, 3H), $5.51(\mathrm{~s}, 1 \mathrm{H}), 3.98(\mathrm{~d}$, $J=17.3 \mathrm{~Hz}, 1 \mathrm{H}), 3.61(\mathrm{~d}, J=17.4 \mathrm{~Hz}, 1 \mathrm{H}) ;{ }^{13} \mathbf{C} \mathbf{N M R}\left(100 \mathrm{MHz}, \mathrm{CDCl}_{3}\right) \delta 198.59$, $137.48,135.00,132.34,129.96,129.67,128.85,128.51,126.24,124.53$ (q, $J=284.9$ $\mathrm{Hz}), 76.48(\mathrm{q}, J=29.1 \mathrm{~Hz}), 40.32 ;{ }^{19} \mathbf{F}$ NMR $\left(376 \mathrm{MHz}, \mathrm{CDCl}_{3}\right) \delta-80.18$; HRMS (ESI) $\mathrm{m} / \mathrm{z}$ calculated for $\mathrm{C}_{16} \mathrm{H}_{12} \mathrm{BrF}_{3} \mathrm{O}_{2}[\mathrm{M}+\mathrm{H}]^{+}: 373.0051$, found: 373.0045 .<smiles>O=C(CC(O)(c1ccccc1)C(F)(F)F)c1ccc(Cl)cc1</smiles>

(3k) Purification via flash column chromatography (silica gel; petroleum ether/ethyl acetate (60 : 1); White solid; $0.15 \mathrm{~g} ;{ }^{1} \mathbf{H}$ NMR (400 MHz, $\left.\mathrm{CDCl}_{3}\right) \delta$ 7.90-7.83 (m, 2H), 7.63-7.56 (m, 2H), 7.52-7.43 (m, 2H), 7.41-7.28 (m, 3H), $5.53(\mathrm{~s}, 1 \mathrm{H}), 3.99$ (d, $J=17.3 \mathrm{~Hz}, 1 \mathrm{H}), 3.62(\mathrm{~d}, J=17.4 \mathrm{~Hz}, 1 \mathrm{H}) ;{ }^{13} \mathbf{C ~ N M R}\left(100 \mathrm{MHz}, \mathrm{CDCl}_{3}\right) \delta 198.38$, $141.15,137.50,134.60,129.63,129.33,128.85,128.51,126.24,124.54$ (q, $J=283.1$ $\mathrm{Hz}), 76.64(\mathrm{q}, J=29.0 \mathrm{~Hz}), 40.32 ;{ }^{19} \mathbf{F}$ NMR $\left(376 \mathrm{MHz}, \mathrm{CDCl}_{3}\right) \delta-80.18$; HRMS (ESI) $\mathrm{m} / \mathrm{z}$ calculated for $\mathrm{C}_{16} \mathrm{H}_{12} \mathrm{ClF}_{3} \mathrm{O}_{2}[\mathrm{M}+\mathrm{H}]^{+}:$329.0556, found: 329.0545 .<smiles>O=C(CC(O)(c1ccccc1)C(F)(F)F)c1cccc(Cl)c1</smiles>

(31) Purification via flash column chromatography (silica gel; petroleum ether/ethyl acetate (60 : 1); Colourless liquid; 0.14 g; ${ }^{1} \mathbf{H}$ NMR (400 MHz, $\left.\mathrm{CDCl}_{3}\right) \delta 7.90-7.85$ (m, 1H), 7.84-7.78 (m, 1H), 7.63-7.55 (m, 3H), 7.48-7.41 (m, 1H), 7.42-7.29 (m, 
3H), $5.43(\mathrm{~s}, 1 \mathrm{H}), 4.00(\mathrm{~d}, J=17.4 \mathrm{~Hz}, 1 \mathrm{H}), 3.63(\mathrm{~d}, J=17.5 \mathrm{~Hz}, 1 \mathrm{H}) ;{ }^{13} \mathbf{C}$ NMR $(100$ $\left.\mathrm{MHz}, \mathrm{CDCl}_{3}\right) \delta 198.30,137.75,137.40,135.41,134.34,130.27,128.86,128.52$, 128.26, 126.29, 126.23, 124.49 (q, $J=282.8 \mathrm{~Hz}), 76.47$ (q, $J=29.0 \mathrm{~Hz}), 40.62 ;{ }^{19} \mathbf{F}$ NMR $\left(376 \mathrm{MHz}, \mathrm{CDCl}_{3}\right) \delta-80.19 ; \mathbf{H R M S}(\mathrm{ESI}) \mathrm{m} / \mathrm{z}$ calculated for $\mathrm{C}_{16} \mathrm{H}_{12} \mathrm{ClF}_{3} \mathrm{O}_{2}$ $[\mathrm{M}+\mathrm{H}]^{+}:$329.0556, found: 329.0542 .

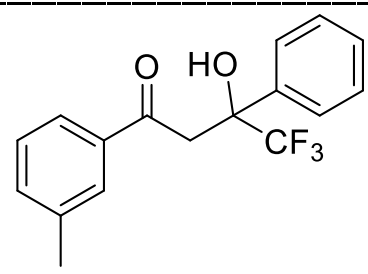

(3m) Purification via flash column chromatography (silica gel; petroleum ether/ethyl acetate (60 : 1); Colourless liquid; $0.14 \mathrm{~g} ;{ }^{1} \mathbf{H}$ NMR (400 MHz, $\left.\mathrm{CDCl}_{3}\right) \delta$ 7.78-7.69 (m, 2H), 7.66-7.57 (m, 2H), 7.49-7.42 (m, 1H), 7.42-7.29 (m, 4H), $5.72(\mathrm{~s}, 1 \mathrm{H}), 4.02$ $(\mathrm{d}, J=17.4 \mathrm{~Hz}, 1 \mathrm{H}), 3.63(\mathrm{~d}, J=17.4 \mathrm{~Hz}, 1 \mathrm{H}), 2.41(\mathrm{~s}, 3 \mathrm{H}) ;{ }^{13} \mathrm{C}$ NMR $(100 \mathrm{MHz}$, $\left.\mathrm{CDCl}_{3}\right) \delta 199.89,138.89,137.74,136.35,135.25,128.81,128.72,128.68,128.43$, $126.33,125.50,124.62(\mathrm{q}, J=283.0 \mathrm{~Hz}), 76.25(\mathrm{q}, J=28.9 \mathrm{~Hz}), 40.23,21.34 ;{ }^{19} \mathbf{F}$ NMR (376 MHz, Chloroform- $d$ ) $\delta$-80.20; HRMS (ESI) $\mathrm{m} / \mathrm{z}$ calculated for $\mathrm{C}_{17} \mathrm{H}_{15} \mathrm{~F}_{3} \mathrm{O}_{2}[\mathrm{M}+\mathrm{Na}]^{+}$: 331.0922, found: 331.0929 .<smiles>O=C(CC(O)(c1ccccc1)C(F)(F)F)c1ccccc1Cl</smiles>

(3n) Purification via flash column chromatography (silica gel; petroleum ether/ethyl acetate (60 : 1); Colourless liquid; $0.14 \mathrm{~g} ;{ }^{1} \mathbf{H}$ NMR (400 MHz, $\left.\mathrm{CDCl}_{3}\right) \delta$ 7.52-7.45 (m, 2H), 7.39-7.32 (m, 2H), 7.32-7.25 (m, 3H), 7.25-7.15 (m, 2H), $5.30(\mathrm{~s}, 1 \mathrm{H})$, 3.96-3.87 (m, 1H), 3.71-3.61 (m, 1H); $\left.{ }^{13} \mathbf{C ~ N M R ~ ( 1 0 0 ~ M H z , ~} \mathrm{CDCl}_{3}\right) \delta$ 202.90, $138.35,137.19,132.97,131.24,130.79$, 129.42, 128.86, 128.43, 127.20, 126.43, $124.46(\mathrm{q}, J=283.1 \mathrm{~Hz}), 76.38(\mathrm{q}, J=29.0 \mathrm{~Hz}), 45.30 ;{ }^{19} \mathbf{F} \mathbf{N M R}\left(376 \mathrm{MHz}, \mathrm{CDCl}_{3}\right)$ $\delta$-80.13; HRMS (ESI) m/z calculated for $\mathrm{C}_{16} \mathrm{H}_{12} \mathrm{ClF}_{3} \mathrm{O}_{2}[\mathrm{M}+\mathrm{Na}]^{+}:$351.0376, found: 351.0370 . 


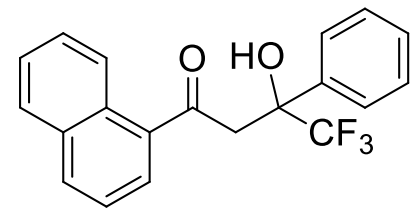

(3o) Purification via flash column chromatography (silica gel; petroleum ether/ethyl acetate (60 : 1); Colourless liquid; $0.14 \mathrm{~g} ;{ }^{1} \mathbf{H}$ NMR (400 MHz, $\left.\mathrm{CDCl}_{3}\right) \delta 8.31-8.23$ (m, 1H), $8.08(\mathrm{~d}, J=8.2 \mathrm{~Hz}, 1 \mathrm{H}), 7.98-7.87(\mathrm{~m}, 2 \mathrm{H}), 7.71-7.63(\mathrm{~m}, 2 \mathrm{H}), 7.60-7.50$ (m, 3H), 7.43-7.32 (m, 3H), 5.89 (s, 1H), 4.07 (d, $J=16.9 \mathrm{~Hz}, 1 \mathrm{H}), 3.82(\mathrm{~d}, J=16.9$ $\mathrm{Hz}, 1 \mathrm{H}) ;{ }^{13} \mathrm{C}$ NMR (100 MHz, $\left.\mathrm{CDCl}_{3}\right) \delta$ 203.73, 137.62, 135.04, 134.22, 133.93, $129.77,128.79,128.56,128.53,128.49,128.39,126.87,126.42,125.30,124.63$ (q, $J$ $=283.1 \mathrm{~Hz}), 124.28,76.89(\mathrm{q}, J=28.8 \mathrm{~Hz}), 43.58 ;{ }^{19} \mathbf{F} \mathbf{N M R}\left(376 \mathrm{MHz}, \mathrm{CDCl}_{3}\right) \delta$ -80.05; HRMS (ESI) $\mathrm{m} / \mathrm{z}$ calculated for $\mathrm{C}_{20} \mathrm{H}_{15} \mathrm{~F}_{3} \mathrm{O}_{2}[\mathrm{M}+\mathrm{Na}]^{+}$: 367.0922, found: 367.0916.

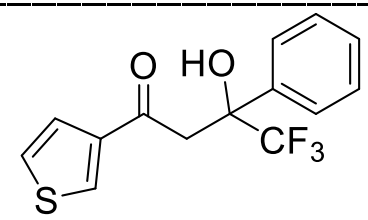

(3p) Purification via flash column chromatography (silica gel; petroleum ether/ethyl acetate (60 : 1); White solid; $0.11 \mathrm{~g} ;{ }^{1} \mathbf{H}$ NMR (400 MHz, $\left.\mathrm{CDCl}_{3}\right) \delta 8.18-8.14(\mathrm{~m}$, 1H), 7.64-7.58 (m, 2H), 7.52-7.48 (m, 1H), 7.43-7.29 (m, 4H), $5.71(\mathrm{~s}, 1 \mathrm{H}), 3.87(\mathrm{~d}$, $J=17.0 \mathrm{~Hz}, 1 \mathrm{H}), 3.59(\mathrm{~d}, J=17.0 \mathrm{~Hz}, 1 \mathrm{H}) ;{ }^{13} \mathbf{C ~ N M R}\left(100 \mathrm{MHz}, \mathrm{CDCl}_{3}\right) \delta 193.42$, $141.63,137.60,133.90,128.77,128.45,127.19,126.63,126.28,124.56$ (q, $J=283.0$ $\mathrm{Hz}), 76.45(\mathrm{q}, J=28.9 \mathrm{~Hz}), 41.51 ;{ }^{19} \mathbf{F}$ NMR $\left(376 \mathrm{MHz}, \mathrm{CDCl}_{3}\right) \delta-80.16$; HRMS (ESI) $\mathrm{m} / \mathrm{z}$ calculated for $\mathrm{C}_{14} \mathrm{H}_{11} \mathrm{~F}_{3} \mathrm{O}_{2} \mathrm{~S}[\mathrm{M}+\mathrm{H}]^{+}:$301.0510, found: 301.0470 .<smiles>CC(O)(CC(=O)CCc1ccccc1)c1ccccc1</smiles>

(3q) Purification via flash column chromatography (silica gel; petroleum ether/ethyl acetate (60 : 1); Colourless liquid; $0.12 \mathrm{~g} ;{ }^{1} \mathbf{H}$ NMR (400 MHz, $\left.\mathrm{CDCl}_{3}\right) \delta 7.50-7.41$ (m, 2H), 7.35-7.24 (m, 3H), 7.23-7.15 (m, 2H), 7.15-7.08 (m, 1H), 7.03-6.96 (m, 2H), $5.37(\mathrm{~s}, 1 \mathrm{H}), 3.31(\mathrm{~d}, J=16.9 \mathrm{~Hz}, 1 \mathrm{H}), 3.15(\mathrm{~d}, J=17.0 \mathrm{~Hz}, 1 \mathrm{H}), 2.88-2.55(\mathrm{~m}$, 4H); ${ }^{13}$ C NMR (100 MHz, $\left.\mathrm{CDCl}_{3}\right) \delta 210.19,139.97,137.39,128.83,128.61,128.49$, 
128.17, 126.38, 126.14, $124.47(\mathrm{q}, J=283.2 \mathrm{~Hz}), 76.07(\mathrm{q}, J=29.1 \mathrm{~Hz}), 46.26,44.76$, 29.72, 28.88; ${ }^{19}$ F NMR $\left(376 \mathrm{MHz}, \mathrm{CDCl}_{3}\right) \delta-80.27$; HRMS (ESI) m/z calculated for $\mathrm{C}_{18} \mathrm{H}_{17} \mathrm{~F}_{3} \mathrm{O}_{2}[\mathrm{M}+\mathrm{H}]^{+}:$323.1259, found: 323.1224.

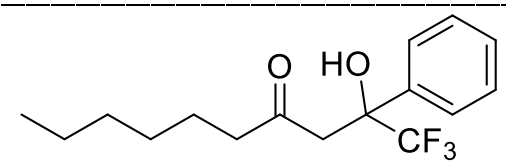

(3r) Purification via flash column chromatography (silica gel; petroleum ether/ethyl acetate (60 : 1); Colourless liquid; $0.13 \mathrm{~g} ;{ }^{1} \mathbf{H}$ NMR (400 MHz, $\left.\mathrm{CDCl}_{3}\right) \delta 7.54-7.44$ (m, 2H), 7.36-7.24 (m, 3H), $5.55(\mathrm{~s}, 1 \mathrm{H}), 3.32(\mathrm{~d}, J=17.0 \mathrm{~Hz}, 1 \mathrm{H}), 3.16(\mathrm{~d}, J=17.0$ $\mathrm{Hz}, 1 \mathrm{H}), 2.48-2.37(\mathrm{~m}, 1 \mathrm{H}), 2.37-2.26(\mathrm{~m}, 1 \mathrm{H}), 1.48-1.36(\mathrm{~m}, 2 \mathrm{H}), 1.23-1.05$ (m, 6H), $0.86(\mathrm{t}, J=6.9 \mathrm{~Hz}, 3 \mathrm{H}) ;{ }^{13} \mathbf{C}$ NMR $\left(100 \mathrm{MHz}, \mathrm{CDCl}_{3}\right) \delta 211.65,137.52,128.78$, $128.43,126.15,124.51(\mathrm{q}, J=283.2 \mathrm{~Hz}), 76.07(\mathrm{q}, J=28.9 \mathrm{~Hz}), 44.98,44.31,31.44$, 28.51, 23.00, 22.42, 14.00; ${ }^{19}$ F NMR (376 MHz, $\left.\mathrm{CDCl}_{3}\right) \delta-80.28 ;$ HRMS (ESI) m/z calculated for $\mathrm{C}_{16} \mathrm{H}_{21} \mathrm{~F}_{3} \mathrm{O}_{2}[\mathrm{M}+\mathrm{Na}]^{+}$: 325.1392, found: 325.1376 .

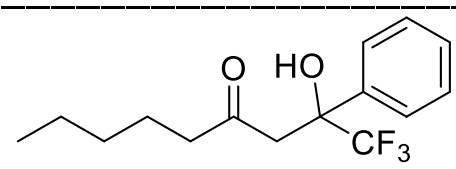

(3s) Purification via flash column chromatography (silica gel; petroleum ether/ethyl acetate (60 : 1); Colourless liquid; $0.12 \mathrm{~g} ;{ }^{1} \mathbf{H}$ NMR (400 MHz, $\left.\mathrm{CDCl}_{3}\right) \delta 7.56(\mathrm{~d}, J=$ 7.4, 2H), 7.45-7.32 (m, 3H), $5.62(\mathrm{~s}, 1 \mathrm{H}), 3.32(\mathrm{~d}, J=16.9 \mathrm{~Hz}, 1 \mathrm{H}), 3.16$ (d, $J=17.0$ $\mathrm{Hz}, 1 \mathrm{H}), 2.56-2.44(\mathrm{~m}, 1 \mathrm{H}), 2.44-2.32(\mathrm{~m}, 1 \mathrm{H}), 1.55-1.45(\mathrm{~m}, 2 \mathrm{H}), 1.30-1.21(\mathrm{~m}$, 2H), $1.21-1.12(\mathrm{~m}, 2 \mathrm{H}), 0.85(\mathrm{t}, J=7.1 \mathrm{~Hz}, 3 \mathrm{H}) ;{ }^{13} \mathrm{C} \mathbf{~ N M R}\left(100 \mathrm{MHz}, \mathrm{CDCl}_{3}\right) \delta$ 211.63, 137.52, 128.78, 128.43, 126.15, 124.50 (q, $J=283.2 \mathrm{~Hz}), 76.07$ (q, $J=29.1$ $\mathrm{Hz}), 44.95,44.32,30.98,22.73,22.33,13.84 ;{ }^{19} \mathbf{F}$ NMR $\left(376 \mathrm{MHz}, \mathrm{CDCl}_{3}\right) \delta-80.28$; HRMS (ESI) m/z calculated for $\mathrm{C}_{15} \mathrm{H}_{19} \mathrm{~F}_{3} \mathrm{O}_{2}[\mathrm{M}+\mathrm{Na}]^{+}:$311.1235, found: 311.1200 .

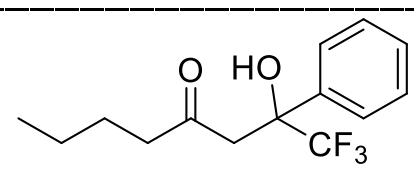

(3t) Purification via flash column chromatography (silica gel; petroleum ether/ethyl acetate (60 : 1); Colourless liquid; $0.12 \mathrm{~g} ;{ }^{1} \mathbf{H}$ NMR (400 MHz, $\left.\mathrm{CDCl}_{3}\right) \delta 7.56(\mathrm{~d}, J=$ $7.3 \mathrm{~Hz}, 2 \mathrm{H}), 7.43-7.32(\mathrm{~m}, 3 \mathrm{H}), 5.63(\mathrm{~s}, 1 \mathrm{H}), 3.32(\mathrm{~d}, J=17.0 \mathrm{~Hz}, 1 \mathrm{H}), 3.16(\mathrm{~d}, J=$ $17.0 \mathrm{~Hz}, 1 \mathrm{H}), 2.56-2.33(\mathrm{~m}, 2 \mathrm{H}), 1.55-1.42(\mathrm{~m}, 2 \mathrm{H}), 1.29-1.16(\mathrm{~m}, 2 \mathrm{H}), 0.85(\mathrm{t}, J=$ 
$7.3 \mathrm{~Hz}, 3 \mathrm{H}) ;{ }^{13} \mathrm{C}$ NMR $\left(100 \mathrm{MHz}, \mathrm{CDCl}_{3}\right) \delta 211.62,137.53,128.78,128.44,126.14$, 124.52 (q, $J=283.2 \mathrm{~Hz}), 76.07$ (q, $J=29.1 \mathrm{~Hz}), 44.68,44.31,25.08,21.98,13.72$; ${ }^{19}$ F NMR $\left(376 \mathrm{MHz}, \mathrm{CDCl}_{3}\right) \delta-80.27$; HRMS (ESI) m/z calculated for $\mathrm{C}_{14} \mathrm{H}_{17} \mathrm{~F}_{3} \mathrm{O}_{2}$ $[\mathrm{M}+\mathrm{H}]^{+}:$275.1259, found: 275.1245 .<smiles>O=C(CC(O)(c1ccc(Br)cc1)C(F)(F)F)c1ccccc1</smiles>

(4a) Purification via flash column chromatography (silica gel; petroleum ether/ethyl acetate (60 : 1); Colourless liquid; $0.16 \mathrm{~g} ;{ }^{\mathbf{1}} \mathbf{H}$ NMR (400 MHz, $\left.\mathrm{CDCl}_{3}\right) \delta$ 7.98-7.89 (m, 2H), 7.69-7.61 (m, 1H), 7.55-7.43 (m, 6H), $5.70(\mathrm{~s}, 1 \mathrm{H}), 4.01(\mathrm{~d}, J=17.4 \mathrm{~Hz}$, $1 \mathrm{H}), 3.63(\mathrm{~d}, J=17.4 \mathrm{~Hz}, 1 \mathrm{H}) ;{ }^{13} \mathrm{C}$ NMR (100 MHz, $\left.\mathrm{CDCl}_{3}\right) \delta 199.51,136.83$, 136.08, 134.69, 131.68, 129.05, 128.27, 128.18, 124.29 (q, $J=282.8 \mathrm{~Hz}$ ), 123.24, 76.34 (q, $J=29.1 \mathrm{~Hz}), 40.00 ;{ }^{19} \mathbf{F}$ NMR $\left(376 \mathrm{MHz}, \mathrm{CDCl}_{3}\right) \delta-80.34$; HRMS (ESI) $\mathrm{m} / \mathrm{z}$ calculated for $\mathrm{C}_{16} \mathrm{H}_{12} \mathrm{BrF}_{3} \mathrm{O}_{2}[\mathrm{M}+\mathrm{H}]^{+}: 373.0051$, found: 373.0026 .<smiles>CC(O)(CC(=O)c1ccccc1)c1ccccc1Br</smiles>

(4b) Purification via flash column chromatography (silica gel; petroleum ether/ethyl acetate (60 : 1); White solid; $0.15 \mathrm{~g} ;{ }^{1} \mathbf{H}$ NMR (400 MHz, $\left.\mathrm{CDCl}_{3}\right) \delta 8.10(\mathrm{~d}, J=7.9$ $\mathrm{Hz}, 1 \mathrm{H}), 7.99$ (d, $J=7.5 \mathrm{~Hz}, 2 \mathrm{H}), 7.69-7.61(\mathrm{~m}, 1 \mathrm{H}), 7.58-7.54(\mathrm{~m}, 1 \mathrm{H}), 7.51(\mathrm{t}, J=$ $7.8 \mathrm{~Hz}, 2 \mathrm{H}), 7.44-7.37(\mathrm{~m}, 1 \mathrm{H}), 7.22-7.14(\mathrm{~m}, 1 \mathrm{H}), 5.53(\mathrm{~s}, 1 \mathrm{H}), 5.13$ (d, J= 18.2 Hz, 1H), $3.72(\mathrm{~d}, J=18.2 \mathrm{~Hz}, 1 \mathrm{H}) ;{ }^{13} \mathbf{C}$ NMR (100 MHz, $\left.\mathrm{CDCl}_{3}\right) \delta$ 199.29, 136.36, $135.71,134.41,131.32,130.43,128.92,128.42,127.64,124.60$ (q, $J=282.8 \mathrm{~Hz}$ ), 120.32, $77.43(\mathrm{q}, J=29.1 \mathrm{~Hz}) 40.91 ;{ }^{19} \mathbf{F}$ NMR $\left(376 \mathrm{MHz}, \mathrm{CDCl}_{3}\right) \delta-78.59 ; \mathbf{H R M S}$ (ESI) $\mathrm{m} / \mathrm{z}$ calculated for $\mathrm{C}_{16} \mathrm{H}_{12} \mathrm{BrF}_{3} \mathrm{O}_{2}[\mathrm{M}+\mathrm{H}]^{+}: 373.0051$, found: 373.0030 .

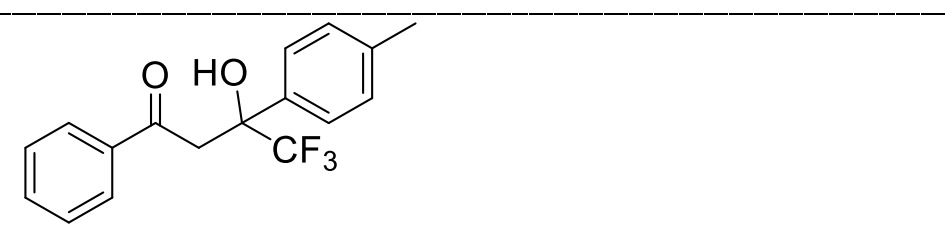


(4c) Purification via flash column chromatography (silica gel; petroleum ether/ethyl acetate (60 : 1); Colourless liquid; $0.14 \mathrm{~g} ;{ }^{1} \mathbf{H}$ NMR (400 MHz, $\left.\mathrm{CDCl}_{3}\right) \delta 7.99-7.88$ (m, 2H), 7.68-7.59 (m, 1H), $7.49(\mathrm{t}, J=8.0, \mathrm{~Hz}, 4 \mathrm{H}), 7.17(\mathrm{~d}, J=8.1 \mathrm{~Hz}, 2 \mathrm{H}), 5.60(\mathrm{~s}$, 1H), $4.05(\mathrm{~d}, J=17.4 \mathrm{~Hz}, 1 \mathrm{H}), 3.61(\mathrm{~d}, J=17.4 \mathrm{~Hz}, 1 \mathrm{H}), 2.32(\mathrm{~s}, 3 \mathrm{H}) ;{ }^{13} \mathbf{C}$ NMR $(100$ $\left.\mathrm{MHz}, \mathrm{CDCl}_{3}\right) \delta 199.71,138.62,136.28,134.66,134.44,129.21,128.95,128.25$, 126.21, $124.60(\mathrm{q}, J=282.7 \mathrm{~Hz}), 76.43(\mathrm{q}, J=28.8 \mathrm{~Hz}), 40.18,21.09 ;{ }^{19}$ F NMR $(376$ $\mathrm{MHz}, \mathrm{CDCl}_{3}$ ) $\delta$-80.44; HRMS (ESI) $\mathrm{m} / \mathrm{z}$ calculated for $\mathrm{C}_{17} \mathrm{H}_{15} \mathrm{~F}_{3} \mathrm{O}_{2}[\mathrm{M}+\mathrm{Na}]^{+}$: 331.0922, found: 331.0910 .<smiles>COc1ccc(C(O)(F)CC(=O)c2ccccc2)cc1</smiles>

(4d) Purification via flash column chromatography (silica gel; petroleum ether/ethyl acetate (60 : 1); Colourless liquid; $0.14 \mathrm{~g} ;{ }^{1} \mathbf{H}$ NMR (400 MHz, $\left.\mathrm{CDCl}_{3}\right) \delta 7.98-7.89$ (m, 2H), 7.68-7.60 (m, 1H), $7.50(\mathrm{t}, J=7.8 \mathrm{~Hz}, 4 \mathrm{H}), 6.92-6.82(\mathrm{~m}, 2 \mathrm{H}), 5.62(\mathrm{~s}, 1 \mathrm{H})$, $4.04(\mathrm{~d}, J=17.4 \mathrm{~Hz}, 1 \mathrm{H}), 3.79(\mathrm{~s}, 3 \mathrm{H}), 3.60(\mathrm{~d}, J=17.4 \mathrm{~Hz}, 1 \mathrm{H}) ;{ }^{13} \mathrm{C}$ NMR $(100$ $\left.\mathrm{MHz}, \mathrm{CDCl}_{3}\right) \delta 199.79,159.79,136.33,134.45,129.58,128.97,128.25,127.64$, $124.62(\mathrm{q}, J=283.0 \mathrm{~Hz}), 113.81,76.28(\mathrm{q}, J=29.0 \mathrm{~Hz}), 55.24,40.09 ;{ }^{19} \mathbf{F}$ NMR $(376$ $\mathrm{MHz}, \mathrm{CDCl}_{3}$ ) $\delta$-80.63; HRMS (ESI) $\mathrm{m} / \mathrm{z}$ calculated for $\mathrm{C}_{17} \mathrm{H}_{15} \mathrm{~F}_{3} \mathrm{O}_{3}[\mathrm{M}+\mathrm{H}]^{+}$: 325.1052, found: 325.1037 .<smiles>COc1ccc(C(C)(O)CC(=O)c2ccccc2)cc1Br</smiles>

(4e) Purification via flash column chromatography (silica gel; petroleum ether/ethyl acetate (60 : 1); Colourless liquid; $0.17 \mathrm{~g} ;{ }^{1} \mathbf{H}$ NMR (400 MHz, $\left.\mathrm{CDCl}_{3}\right) \delta 8.01-7.90$ (m, 2H), 7.82-7.76 (m, 1H), 7.70-7.59 (m, 1H), 7.58-7.45 (m, 3H), $6.86(\mathrm{~d}, J=8.7$ $\mathrm{Hz}, 1 \mathrm{H}), 5.71(\mathrm{~s}, 1 \mathrm{H}), 3.96(\mathrm{~d}, J=17.4 \mathrm{~Hz}, 1 \mathrm{H}), 3.87$ (s, 3H), 3.62 (d, J = $17.4 \mathrm{~Hz}$, 1H); ${ }^{13}$ C NMR (100 MHz, $\left.\mathrm{CDCl}_{3}\right) \delta 199.59,156.11,136.12,134.63,131.52,131.09$, $129.02,128.28,126.63,124.42(\mathrm{q}, J=282.9 \mathrm{~Hz}), 111.82,111.46,75.87(\mathrm{q}, J=29.1$ 
$\mathrm{Hz}), 56.24,39.98 ;{ }^{19} \mathbf{F}$ NMR (376 $\left.\mathrm{MHz}, \mathrm{CDCl}_{3}\right) \delta-80.48 ; \mathbf{H R M S}(\mathrm{ESI}) \mathrm{m} / \mathrm{z}$ calculated for $\mathrm{C}_{17} \mathrm{H}_{14} \mathrm{BrF}_{3} \mathrm{O}_{3}[\mathrm{M}+\mathrm{H}]^{+}$: 403.0157, found: 403.0139 .<smiles>O=C(CC(O)(c1ccc(Br)c([N+](=O)[O-])c1)C(F)(F)F)c1ccccc1</smiles>

(4f) Purification via flash column chromatography (silica gel; petroleum ether/ethyl acetate (60 : 1); Pale yellow solid; 0.16 g; ${ }^{1} \mathbf{H}$ NMR (400 MHz, $\mathrm{CDCl}_{3}$ ) $\delta$ 8.17-8.08 (m, 1H), $7.94(\mathrm{~d}, J=7.7 \mathrm{~Hz}, 2 \mathrm{H}), 7.75(\mathrm{~d}, J=8.5 \mathrm{~Hz}, 1 \mathrm{H}), 7.72-7.64(\mathrm{~m}, 2 \mathrm{H}), 7.53$ (t, $J=7.8 \mathrm{~Hz}, 2 \mathrm{H}), 5.89(\mathrm{~s}, 1 \mathrm{H}), 3.94(\mathrm{~d}, J=17.5 \mathrm{~Hz}, 1 \mathrm{H}), 3.74(\mathrm{~d}, J=17.5 \mathrm{~Hz}, 1 \mathrm{H}) ;{ }^{13} \mathrm{C}$ NMR $\left(100 \mathrm{MHz}, \mathrm{CDCl}_{3}\right) \delta 199.07,149.76,139.24,135.66,135.34,135.08,131.08$, 129.17, 128.35, 124.07, 123.89 (q, $J=283.3 \mathrm{~Hz}$ ), 115.38, 76.00 (q, $J=29.6 \mathrm{~Hz}$ ), 39.77; ${ }^{19} \mathbf{F}$ NMR $\left(376 \mathrm{MHz}, \mathrm{CDCl}_{3}\right) \delta$-80.07; HRMS (ESI) $\mathrm{m} / \mathrm{z}$ calculated for $\mathrm{C}_{16} \mathrm{H}_{11} \mathrm{BrF}_{3} \mathrm{NO}_{4}[\mathrm{M}+\mathrm{H}]^{+}$: 417.9902 , found: 417.9889 .<smiles>COc1ccc(C(F)(F)F)c(OC)c1OCC(=O)c1ccccc1</smiles>

(4g) Purification via flash column chromatography (silica gel; petroleum ether/ethyl acetate (60 : 1); Colourless liquid; $0.17 \mathrm{~g}$; ${ }^{1} \mathbf{H}$ NMR (400 MHz, $\left.\mathrm{CDCl}_{3}\right) \delta 7.95$ (d, $J=$ $7.6 \mathrm{~Hz}, 2 \mathrm{H}), 7.65-7.54(\mathrm{~m}, 1 \mathrm{H}), 7.53-7.40(\mathrm{~m}, 3 \mathrm{H}), 6.71(\mathrm{~d}, J=9.0 \mathrm{~Hz}, 1 \mathrm{H}), 5.85$ (s, $1 \mathrm{H}), 4.47(\mathrm{~d}, J=17.1 \mathrm{~Hz}, 1 \mathrm{H}), 3.91-3.83(\mathrm{~m}, 6 \mathrm{H}), 3.70(\mathrm{~s}, 3 \mathrm{H}), 3.57$ (d, $J=17.1 \mathrm{~Hz}$, 1H); ${ }^{13} \mathbf{C ~ N M R}\left(100 \mathrm{MHz}, \mathrm{CDCl}_{3}\right) \delta$ 199.02, 154.20, 151.96, 141.87, 136.70, 133.82, $128.68,128.27,124.72(\mathrm{q}, J=283.4 \mathrm{~Hz}), 124.12,121.41,106.82,76.45$ (q, $J=29.3$ $\mathrm{Hz}), 61.03,60.47,55.85,41.03 ;{ }^{19}$ F NMR $\left(376 \mathrm{MHz}, \mathrm{CDCl}_{3}\right) \delta-80.82$; HRMS (ESI) $\mathrm{m} / \mathrm{z}$ calculated for $\mathrm{C}_{19} \mathrm{H}_{19} \mathrm{~F}_{3} \mathrm{O}_{5}[\mathrm{M}+\mathrm{H}]^{+}:$385.1263, found: 385.1241 . 


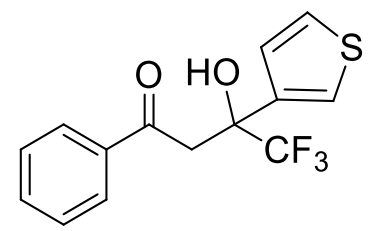

(4h) Purification via flash column chromatography (silica gel; petroleum ether/ethyl acetate (60 : 1); Colourless liquid; $0.11 \mathrm{~g} ;{ }^{1} \mathbf{H}$ NMR (400 MHz, $\left.\mathrm{CDCl}_{3}\right) \delta 8.01-7.90$ (m, 2H), 7.70-7.61 (m, 1H), $7.51(\mathrm{t}, J=7.8 \mathrm{~Hz}, 2 \mathrm{H}), 7.35-7.28(\mathrm{~m}, 1 \mathrm{H}), 7.15-7.08$ (m, 1H), 7.01-6.93 (m, 1H), $6.21(\mathrm{~s}, 1 \mathrm{H}), 3.97(\mathrm{~d}, J=17.2 \mathrm{~Hz}, 1 \mathrm{H}), 3.59$ (d, $J=17.2$ $\mathrm{Hz}, 1 \mathrm{H}) ;{ }^{13} \mathrm{C}$ NMR $\left(100 \mathrm{MHz}, \mathrm{CDCl}_{3}\right) \delta 199.63,141.94,136.13,134.62,129.00$, 128.34, 127.19, 126.60, 125.89, 123.91 (q, $J=282.7 \mathrm{~Hz}), 75.90(\mathrm{q}, J=30.2 \mathrm{~Hz})$, 40.83; ${ }^{19}$ F NMR $\left(376 \mathrm{MHz}, \mathrm{CDCl}_{3}\right) \delta$-81.29; HRMS (ESI) m/z calculated for $\mathrm{C}_{14} \mathrm{H}_{11} \mathrm{~F}_{3} \mathrm{O}_{2} \mathrm{~S}[\mathrm{M}+\mathrm{H}]^{+}$: 301.0510, found: 301.0498 .<smiles>O=C(/C=C(/c1ccccc1)C(F)(F)F)c1ccccc1</smiles>

(3a'-E) Purification via flash column chromatography (silica gel; petroleum ether/ethyl acetate (80 : 1); Pale yellow solid; $85 \mathrm{mg}$ (0.5 mmol scale); ${ }^{1}$ H NMR (400 $\left.\mathrm{MHz}, \mathrm{CDCl}_{3}\right) \delta$ 7.84-7.79 (m, 2H), 7.55-7.50 (m, 1H), 7.42-7.35 (m, 2H), 7.30-7.24 $(\mathrm{m}, 6 \mathrm{H}) ;{ }^{13} \mathbf{C} \mathbf{N M R}\left(100 \mathrm{MHz}, \mathrm{CDCl}_{3}\right) \delta 192.01,138.85(\mathrm{q}, J=30.6 \mathrm{~Hz}), 136.04$, 133.84, 130.82 (q, $J=5.0 \mathrm{~Hz}), 130.79,129.36,129.01,128.88,128.65,128.32$, $122.84(\mathrm{q}, J=273.1 \mathrm{~Hz}) ;{ }^{19}$ F NMR $\left(376 \mathrm{MHz} \mathrm{CDCl}_{3}\right) \delta-66.26 ; \mathbf{H R M S}(\mathrm{ESI}) \mathrm{m} / \mathrm{z}$ calculated for $\mathrm{C}_{16} \mathrm{H}_{12} \mathrm{~F}_{3} \mathrm{O}[\mathrm{M}+\mathrm{H}]^{+}$: 277.0840, found: 277.0828 .<smiles>O=C(C=C(c1ccccc1)C(F)(F)F)c1ccccc1</smiles>

(3a'-Z) Purification via flash column chromatography (silica gel; petroleum ether/ethyl acetate (80 : 1); Pale yellow solid; $28 \mathrm{mg}$ (0.5 mmol scale); ${ }^{1}$ H NMR (400 
$\left.\mathrm{MHz}, \mathrm{CDCl}_{3}\right) \delta$ 8.04-7.97 (m, 2H), 7.69-7.61 (m, 1H), 7.59-7.50 (m, 4H), 7.50-7.42

(m, 3H), $6.84(\mathrm{~s}, 1 \mathrm{H}) ;{ }^{13} \mathbf{C}$ NMR $\left(100 \mathrm{MHz}, \mathrm{CDCl}_{3}\right) \delta 192.43,135.91$ (q, $\left.J=31.1 \mathrm{~Hz}\right)$, $135.65,134.42(\mathrm{q}, J=3.5 \mathrm{~Hz}), 134.17,133.52,129.55,129.14,129.02,128.85$, $128.79,127.80,122.63$ (q, $J=274.5 \mathrm{~Hz}) ;{ }^{19} \mathbf{F}$ NMR $\left(376 \mathrm{MHz}, \mathrm{CDCl}_{3}\right) \delta$-59.25; HRMS (ESI) m/z calculated for $\mathrm{C}_{16} \mathrm{H}_{12} \mathrm{~F}_{3} \mathrm{O}[\mathrm{M}+\mathrm{H}]^{+}:$277.0840, found: 277.0830.

\section{Crystallography of compound $\mathbf{3 j}$}

Single-crystal X-ray diffraction data for the reported complex was recorded at a temperature of 293(2) K on a Oxford Diffraction Gemini R Ultra diffractometer, using a $\omega$ scan technique with Mo-K $\alpha$ radiation $(\lambda=0.71073 \AA$ ). The structure was solved by Direct Method of SHELXS-97 and refined by full-matrix least-squares techniques using the SHELXL-97 program.1 Non-hydrogen atoms were refined with anisotropic temperature parameters, and hydrogen atoms of the ligands were refined as rigid groups. Basic information pertaining to crystal parameters and structure refinement is summarized in Table 1. 1 (a) G. M. Sheldrick, SHELXS-97, Program for Solution of Crystal Structures, University of Gottingen, Germany, 1997; (b) G. M. Sheldrick, SHELXL-97, Program for Refinement of Crystal Structures, University of Gottingen, Germany, 1997.
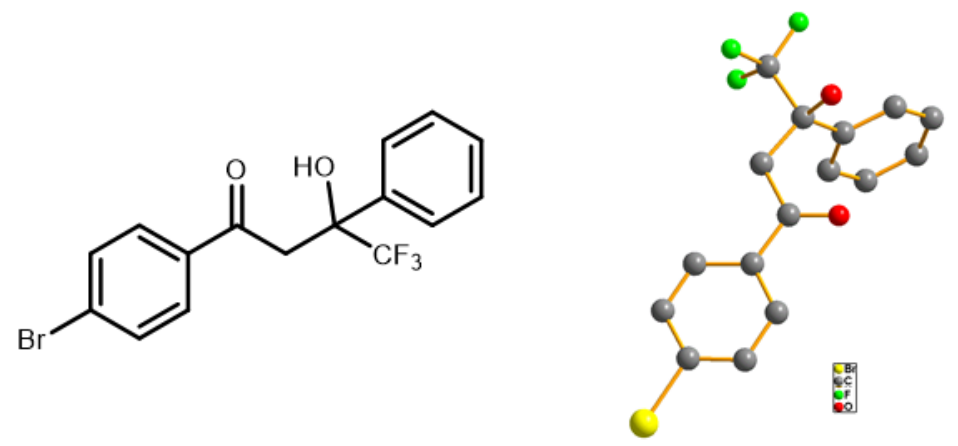

Table 1. Crystal data and structure refinement. 


\begin{tabular}{|c|c|}
\hline Empirical formula & $\mathrm{C}_{16} \mathrm{H}_{12} \mathrm{BrF}_{3} \mathrm{O}_{2}$ \\
\hline Temperature & $293(2) \mathrm{K}$ \\
\hline Wavelength & $1.54178 \AA$ \\
\hline Unit cell dimensions & $\begin{array}{c}\mathrm{a}=11.2170(8) \AA \quad \text { alpha }=90 \mathrm{deg} . \\
\mathrm{b}=6.4731(4) \AA \quad \text { beta }=96.814(6) \mathrm{deg} . \\
\mathrm{c}=21.0339(14) \AA \quad \text { gamma }=90 \mathrm{deg} .\end{array}$ \\
\hline Volume & $1516.45(17) \AA^{3}$ \\
\hline$Z$ & 4 \\
\hline Calculated density & $1.634 \mathrm{Mg} / \mathrm{m}^{3}$ \\
\hline Absorption coefficient & $4.048 \mathrm{~mm} \mathrm{~m}^{-1}$ \\
\hline F(000) & 744.0 \\
\hline Crystal size & $0.39 \times 0.05 \times 0.03 \mathrm{~mm}$ \\
\hline Theta range for data collection & 8.46 to $134.1 \mathrm{deg}$. \\
\hline Reflections collected / unique & $5061 / 2709\left[\mathrm{R}_{\text {in }} \mathrm{t}=0.0259\right]$ \\
\hline Data / restraints / parameters & $2709 / 0 / 201$ \\
\hline Goodness-of-fit on $\mathrm{F}^{2}$ & 1.055 \\
\hline Final $\mathrm{R}$ indices [I>2sigma(I)] & $\mathrm{R}_{1}=0.0430, \mathrm{wR}_{2}=0.1042$ \\
\hline $\mathrm{R}$ indices (all data) & $\mathrm{R}_{1}=0.0718, \mathrm{wR} 2=0.1312$ \\
\hline
\end{tabular}

\section{NMR spectra copies}


<smiles>CC(C)(C)C(C(=O)c1ccccc1)c1ccccc1</smiles>

3a

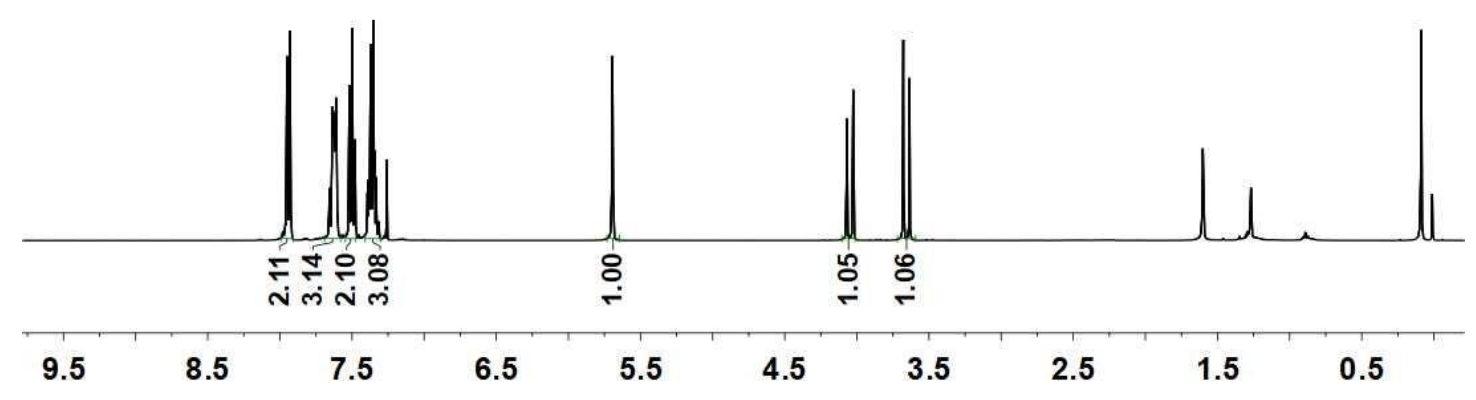

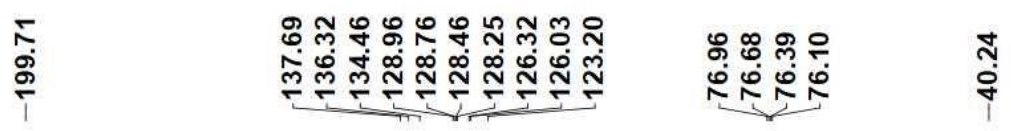<smiles>O=C(c1ccccc1)C1(O)C(c2ccccc2)C(F)(F)C1(F)F</smiles>

3a

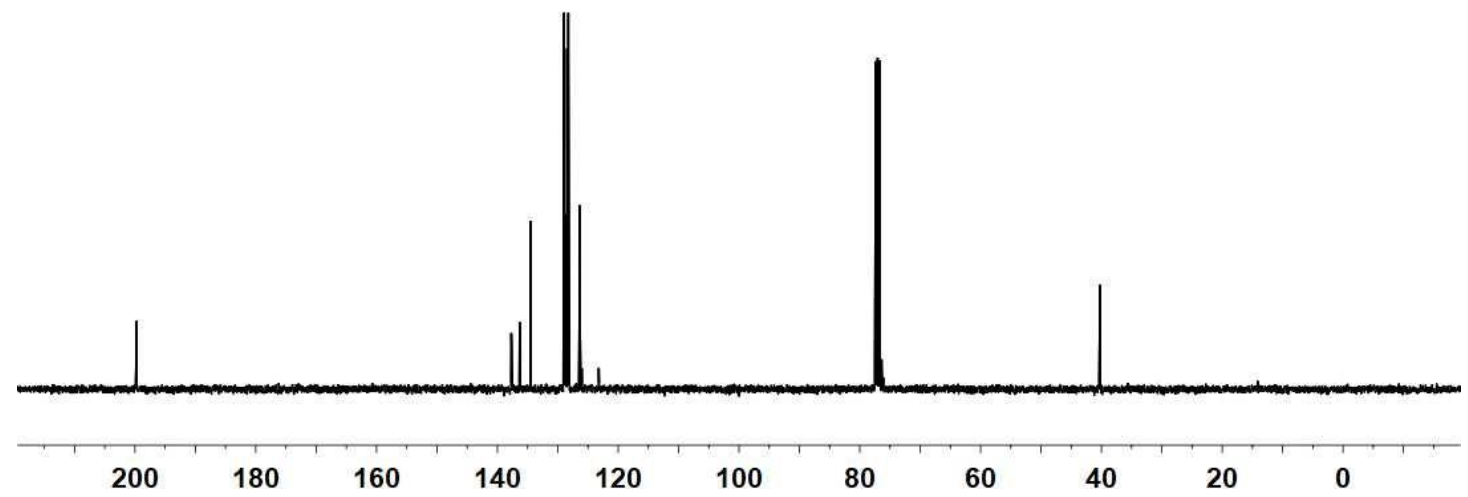




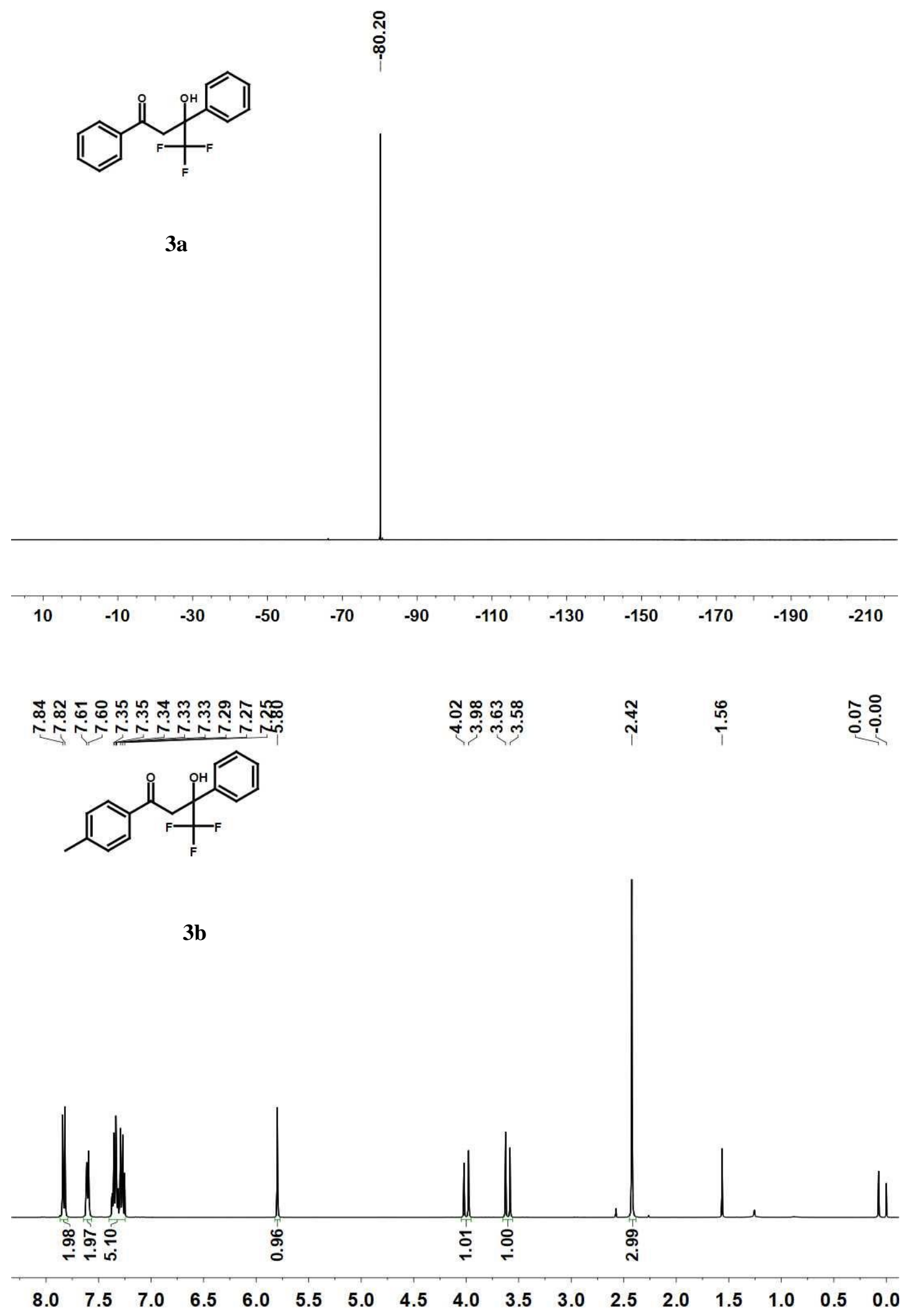


<smiles>Cc1ccc(C(=O)CC(O)(c2ccccc2)C(F)(F)F)cc1</smiles>

3b

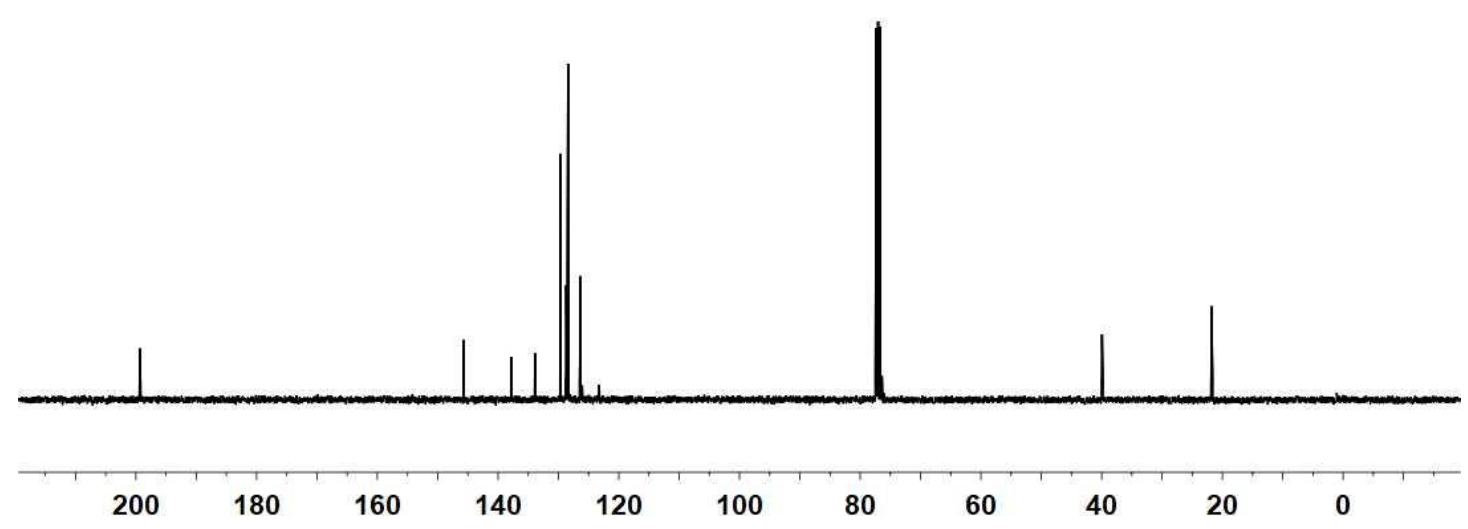<smiles>Cc1ccc(C(=O)CC(O)(c2ccccc2)C(F)(F)F)cc1</smiles>

3b

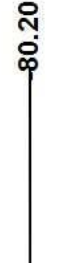

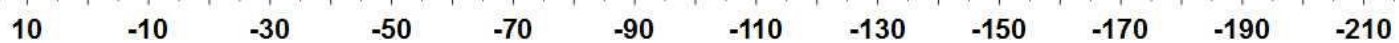


<smiles>CCc1ccc(C(=O)CC(O)(c2ccccc2)C(F)(F)F)cc1</smiles>

3c

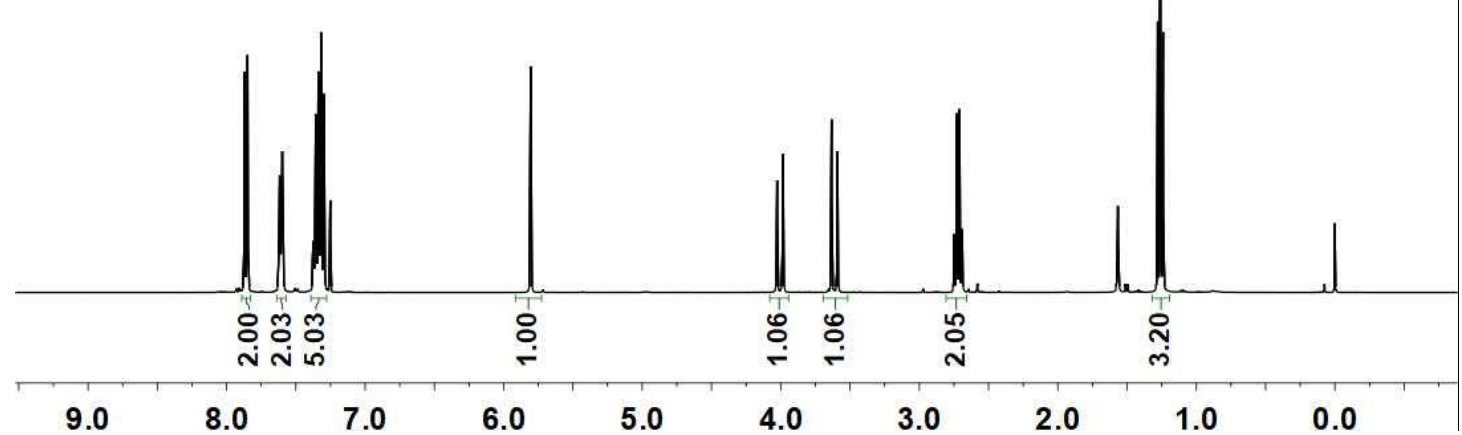
ตั

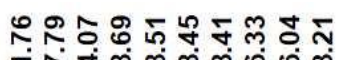

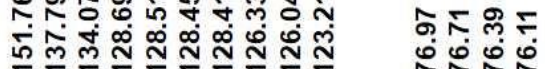

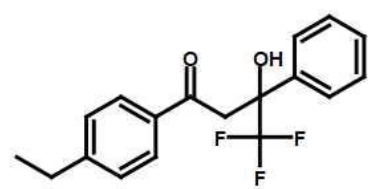

3c

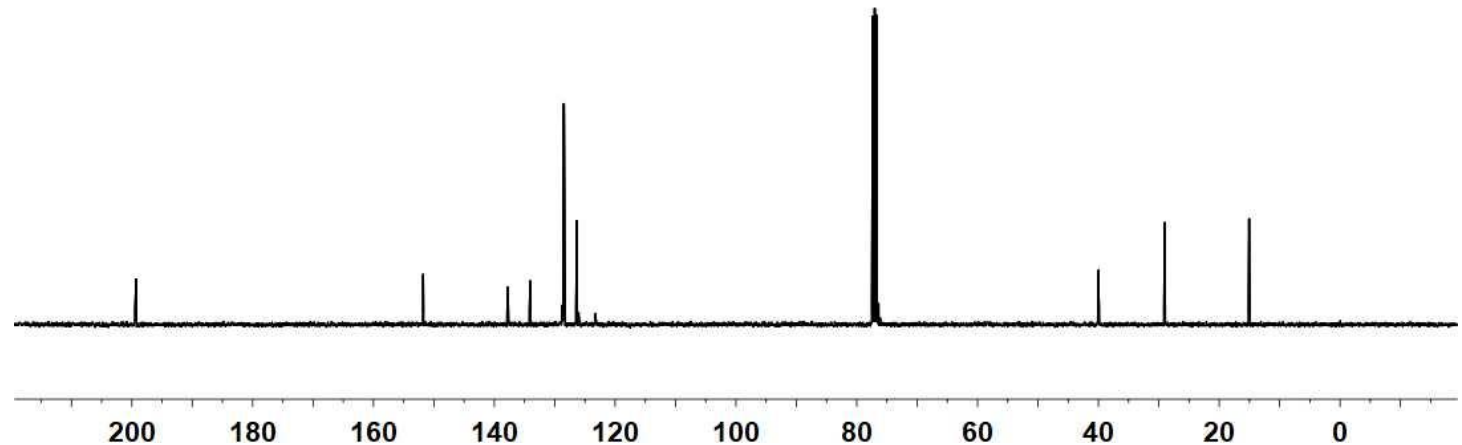




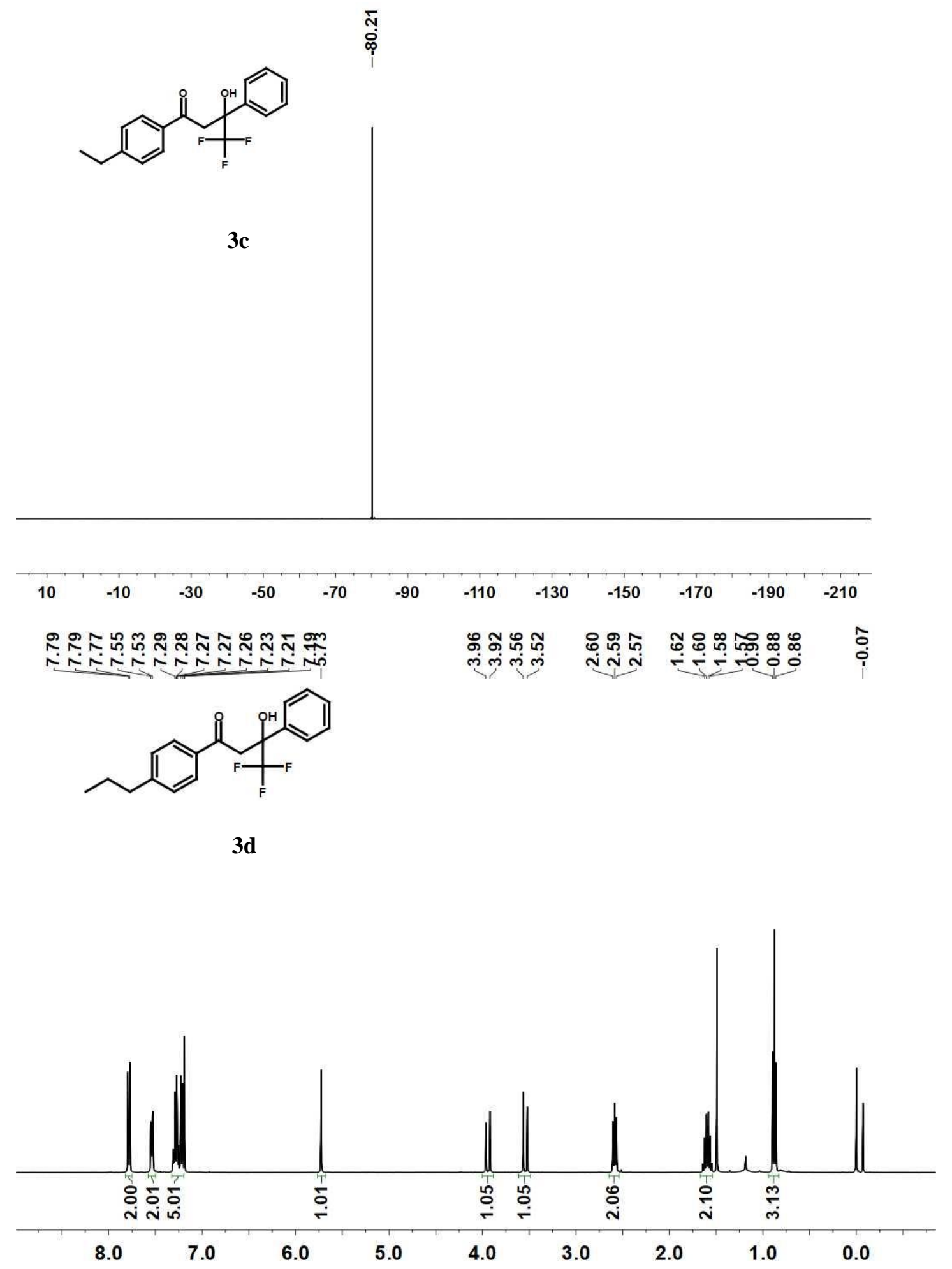


ஸั

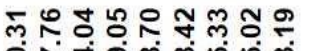

ผึ่

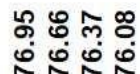

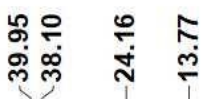

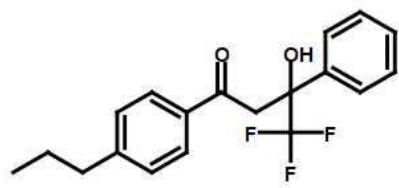

3d

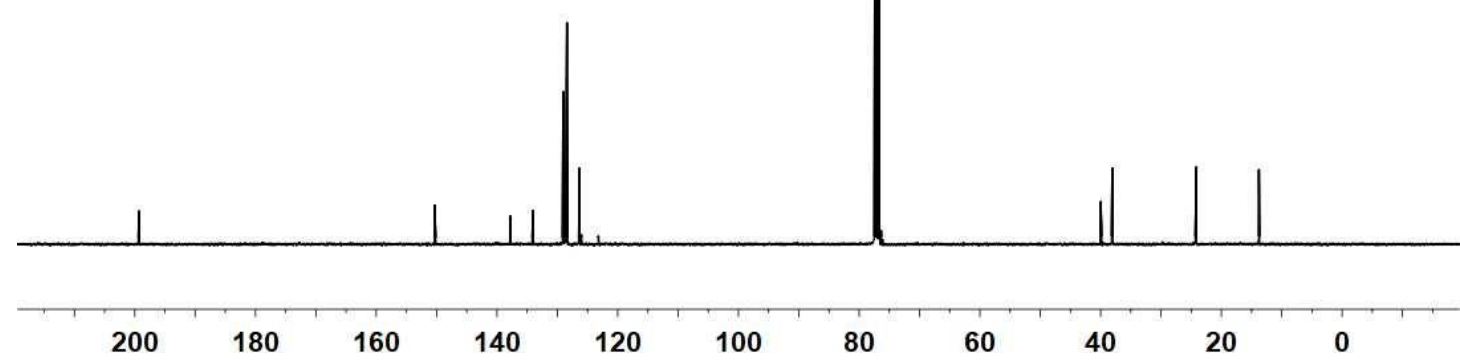

\ั)<smiles>CCCc1ccc(C(=O)CC(O)(c2ccccc2)C(F)(F)F)cc1</smiles>

3d 


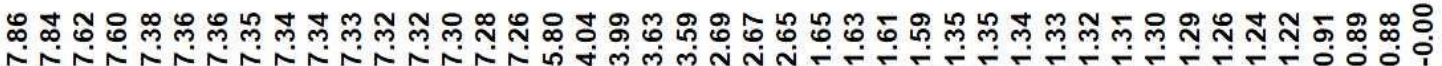<smiles>CCCCCc1ccc(C(=O)CC(O)(c2ccccc2)C(F)(F)F)cc1</smiles>

$3 e$

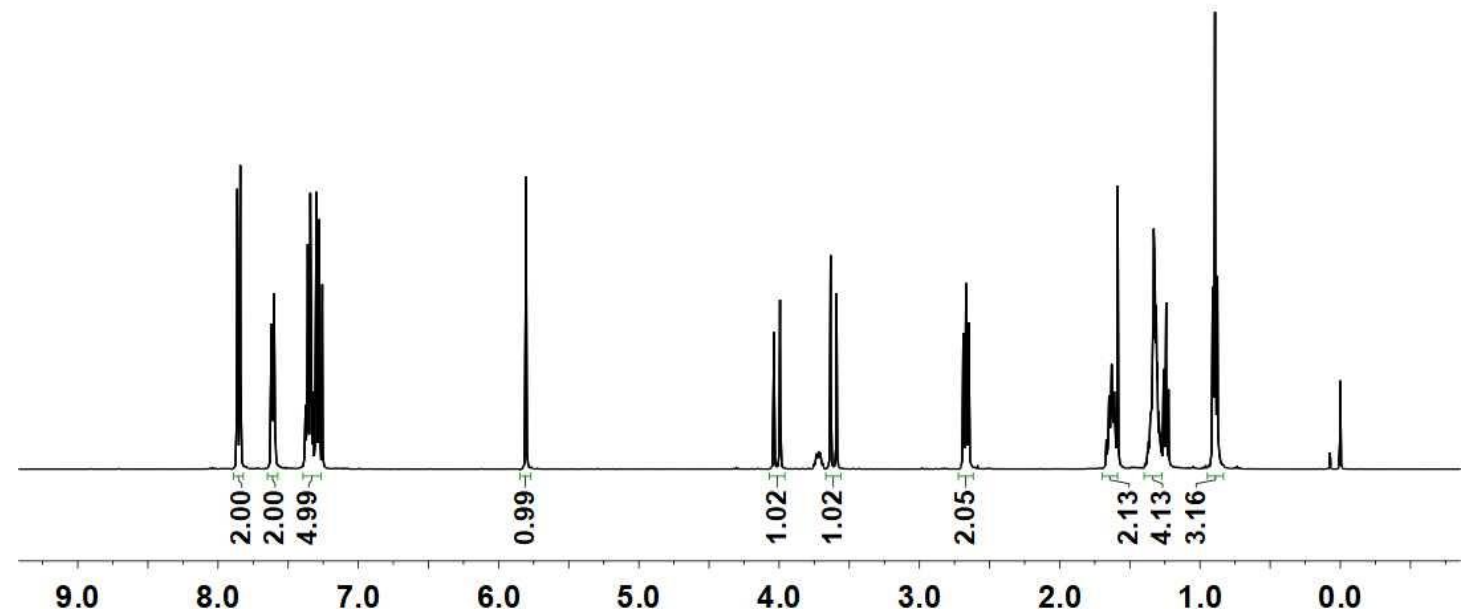

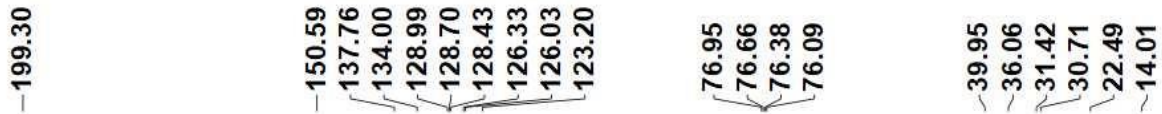

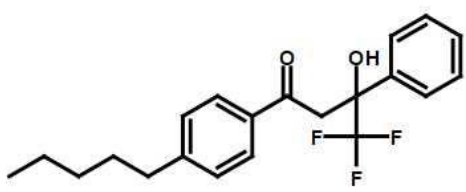

$3 e$

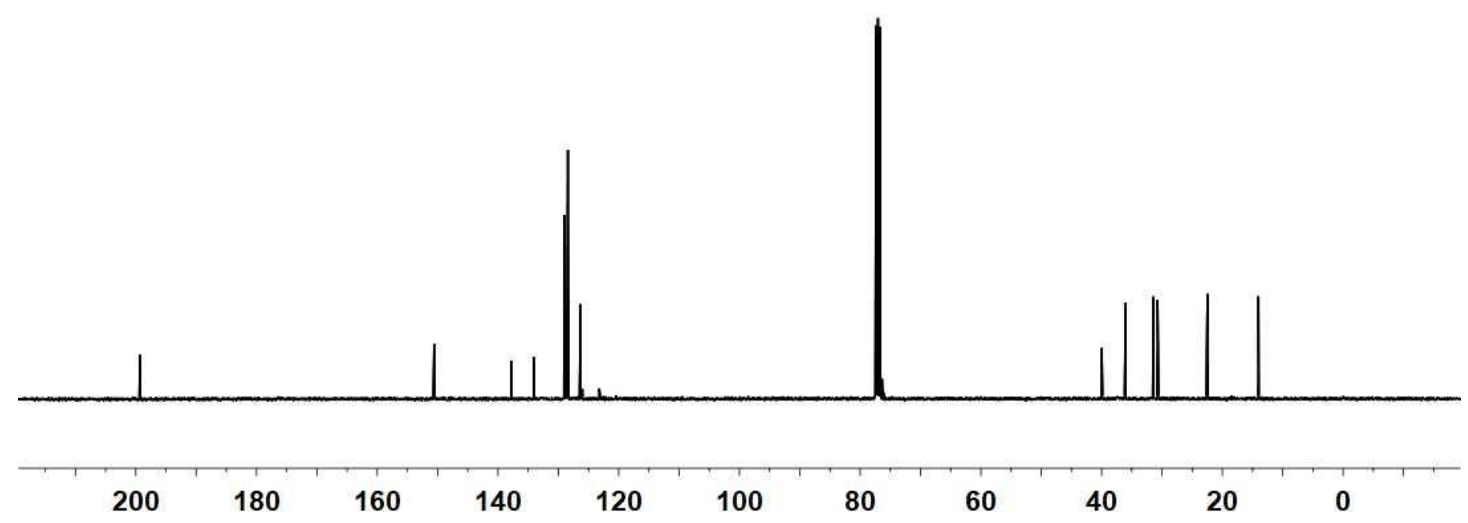




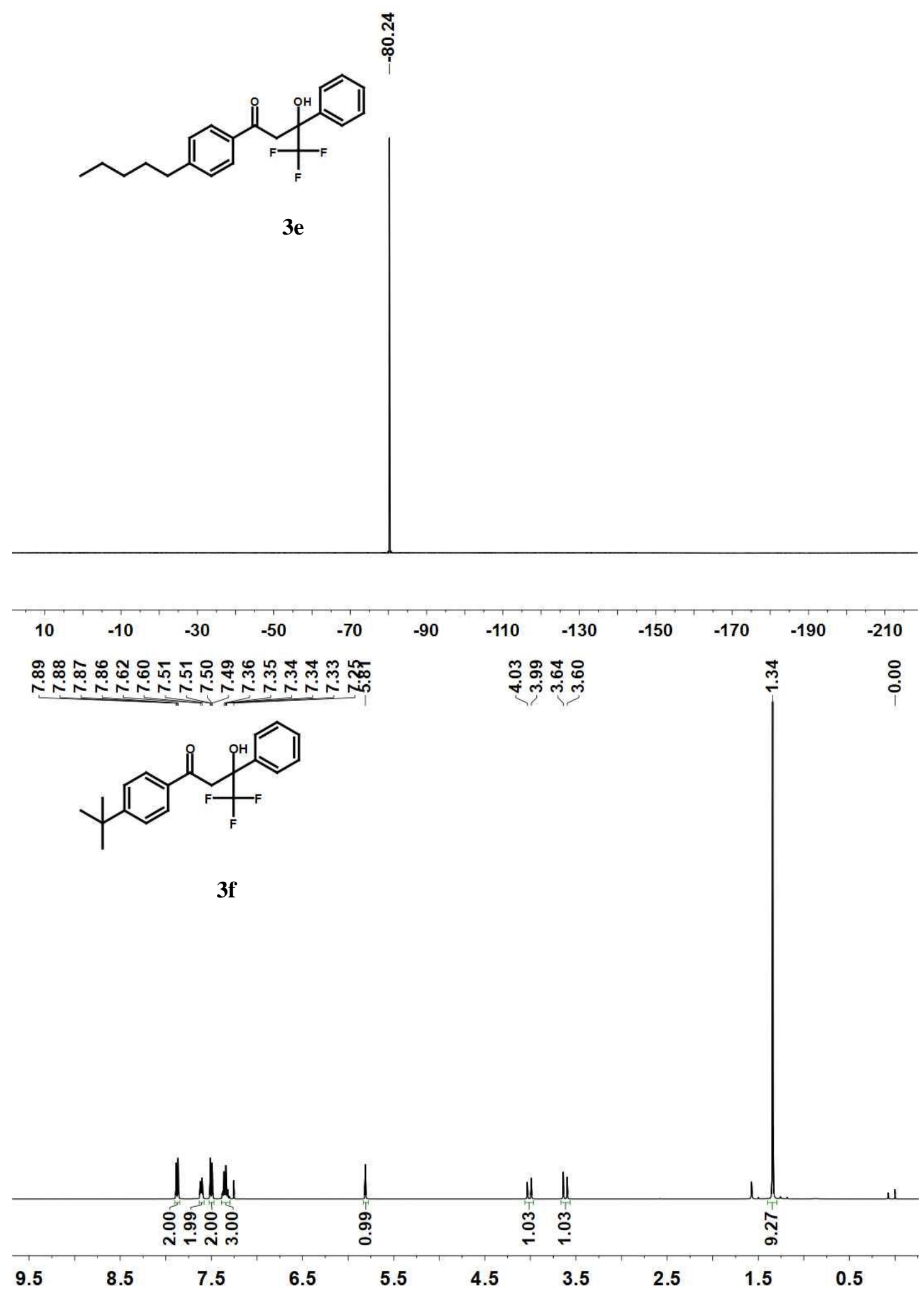


ஓ.

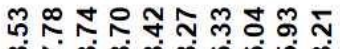

吕

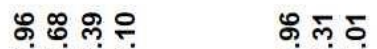

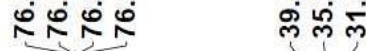<smiles>CC(C)(C)c1ccc(C(=O)CC(O)(c2ccccc2)C(F)(F)F)cc1</smiles>

3f
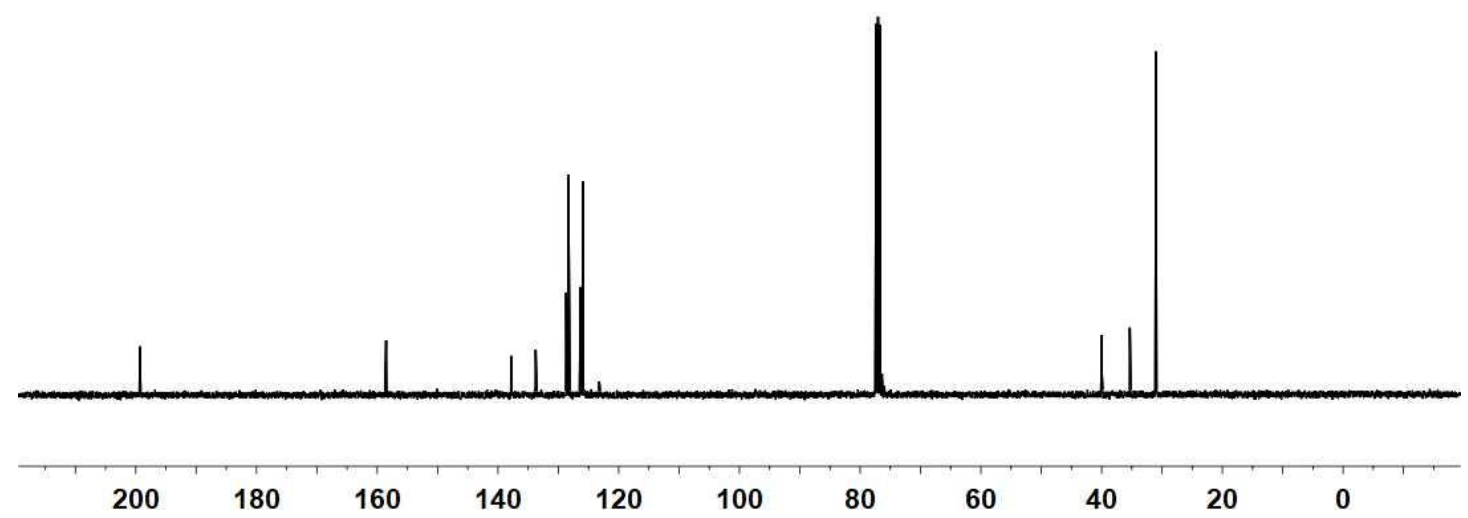

พิ<smiles>CC(C)(C)c1ccc(C(=O)CC(O)(c2ccccc2)C(F)(F)F)cc1</smiles>

3f 


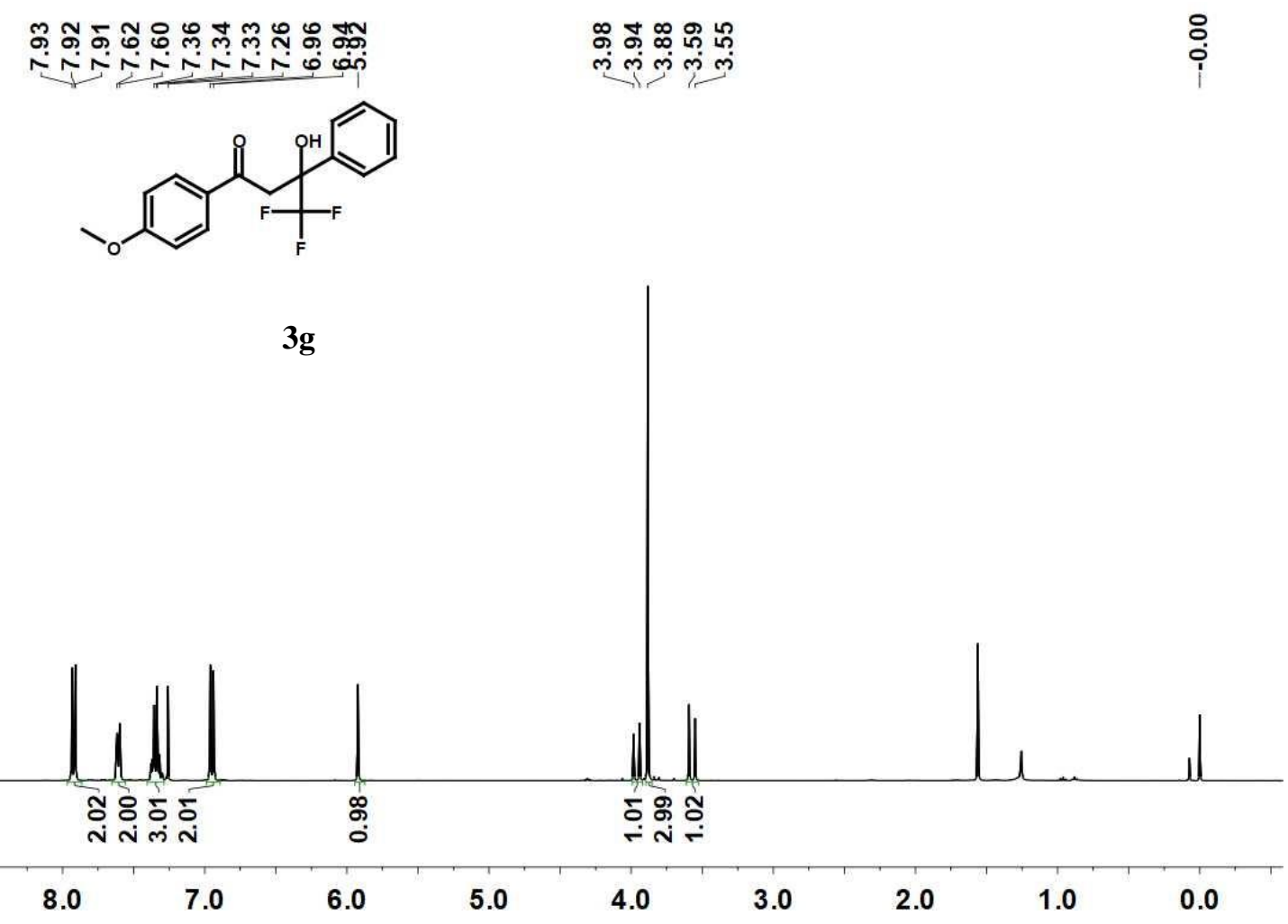

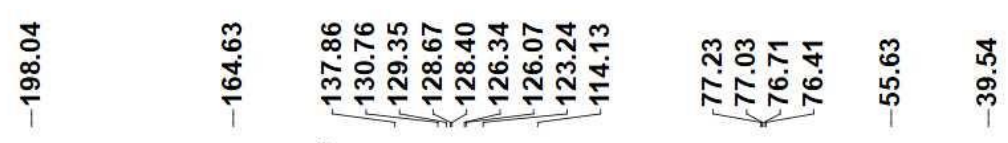<smiles>COc1ccc(C(=O)CC(O)(c2ccccc2)C(F)(F)F)cc1</smiles>

$3 g$

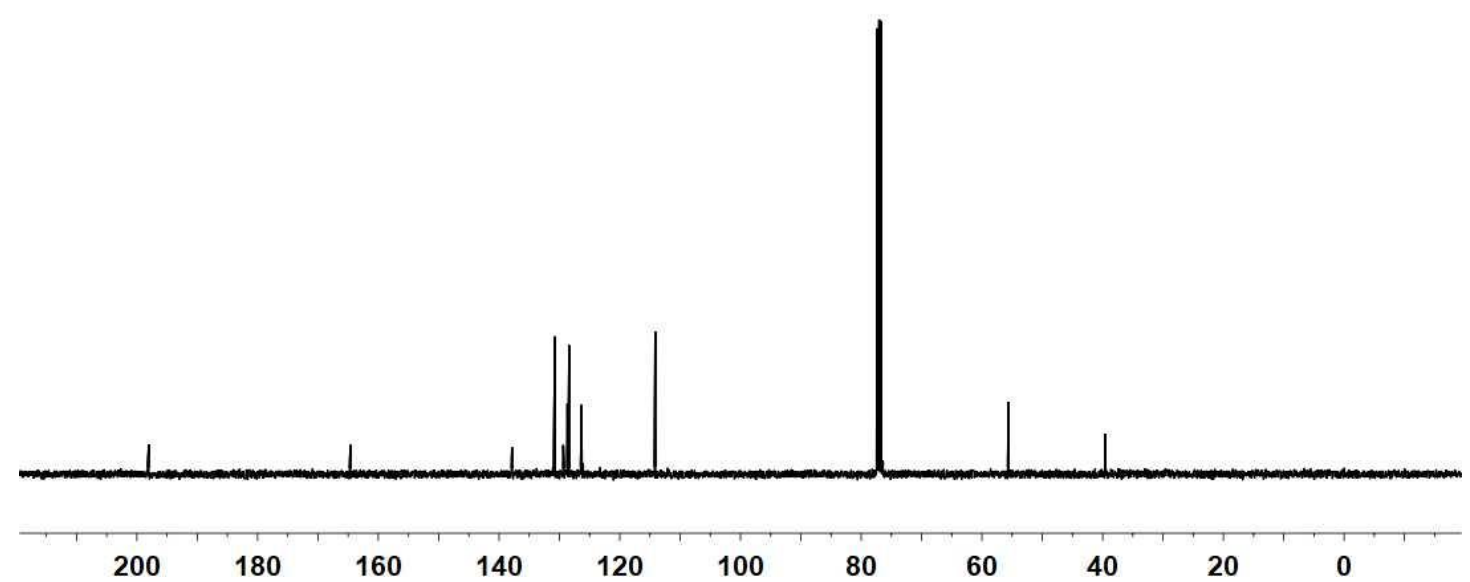




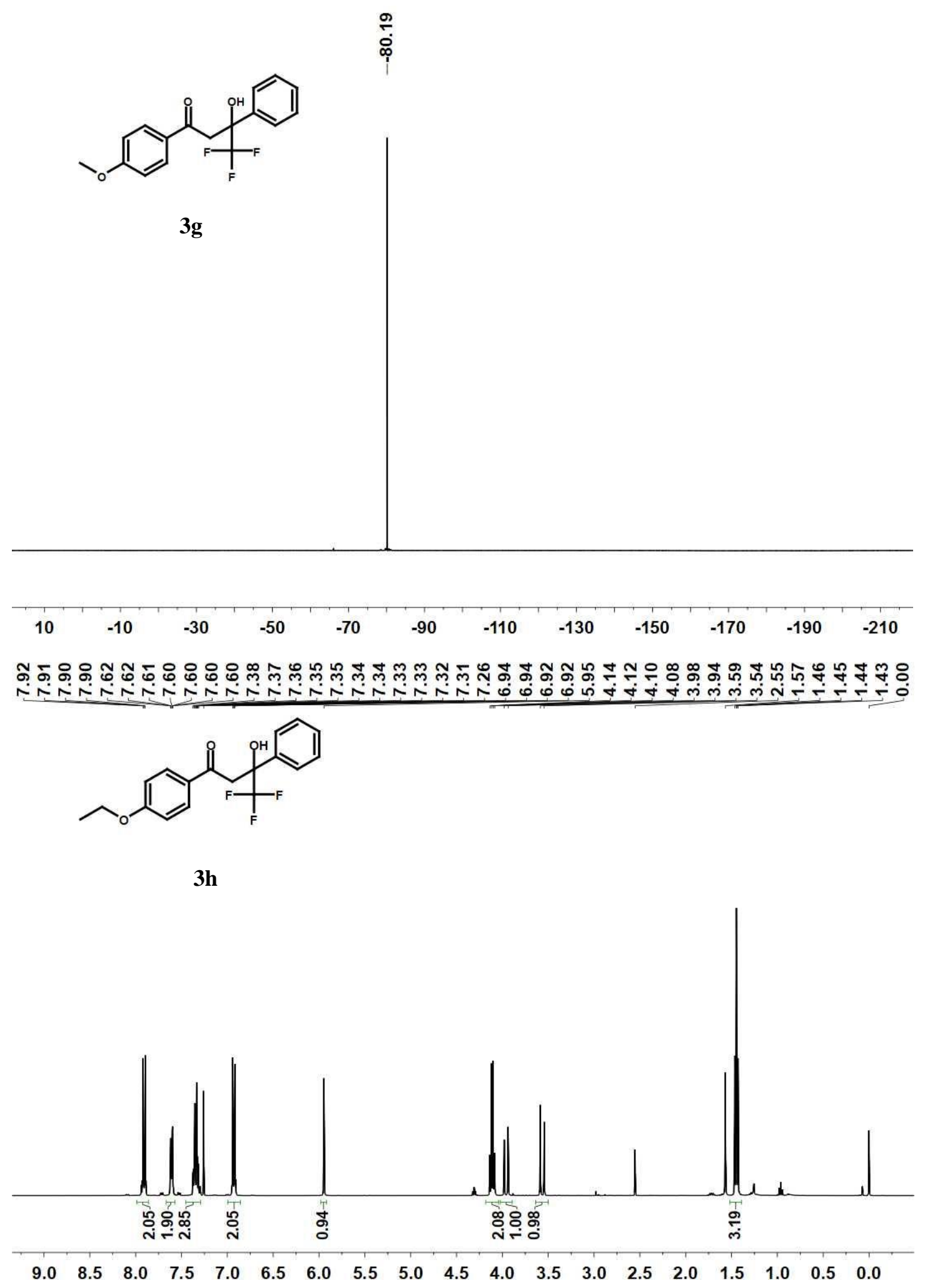




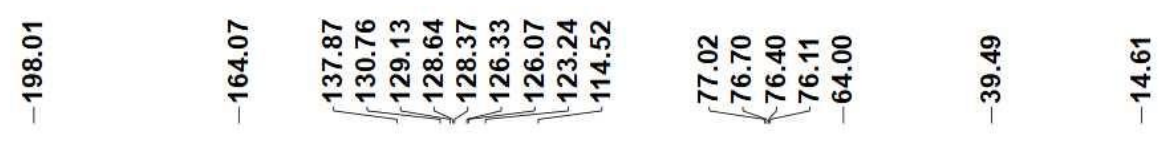

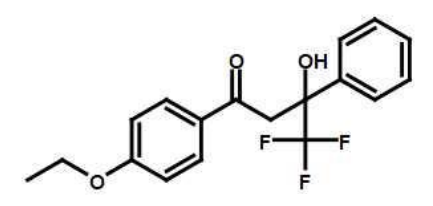

3h

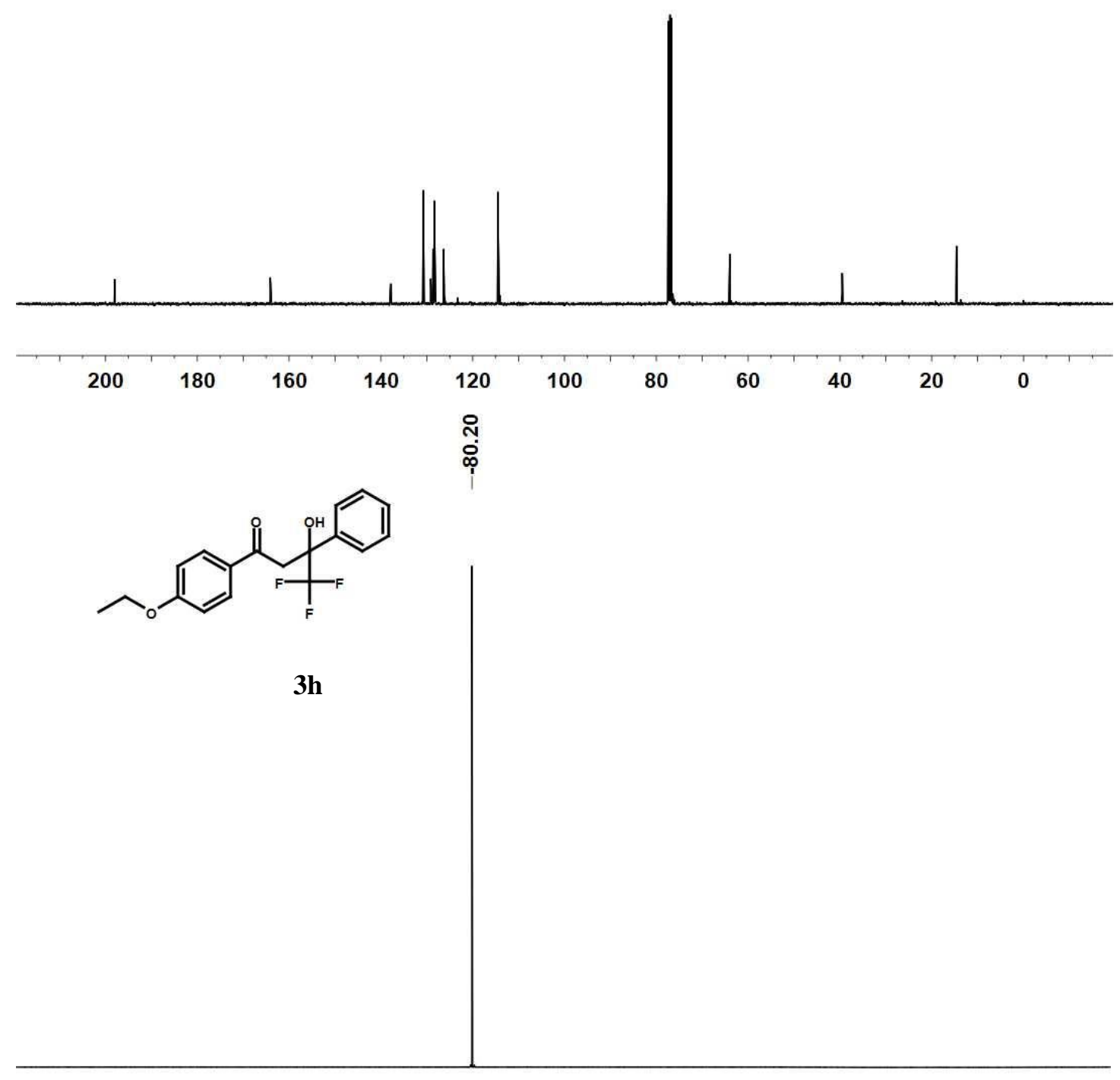

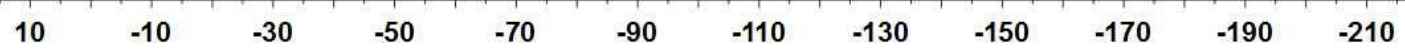




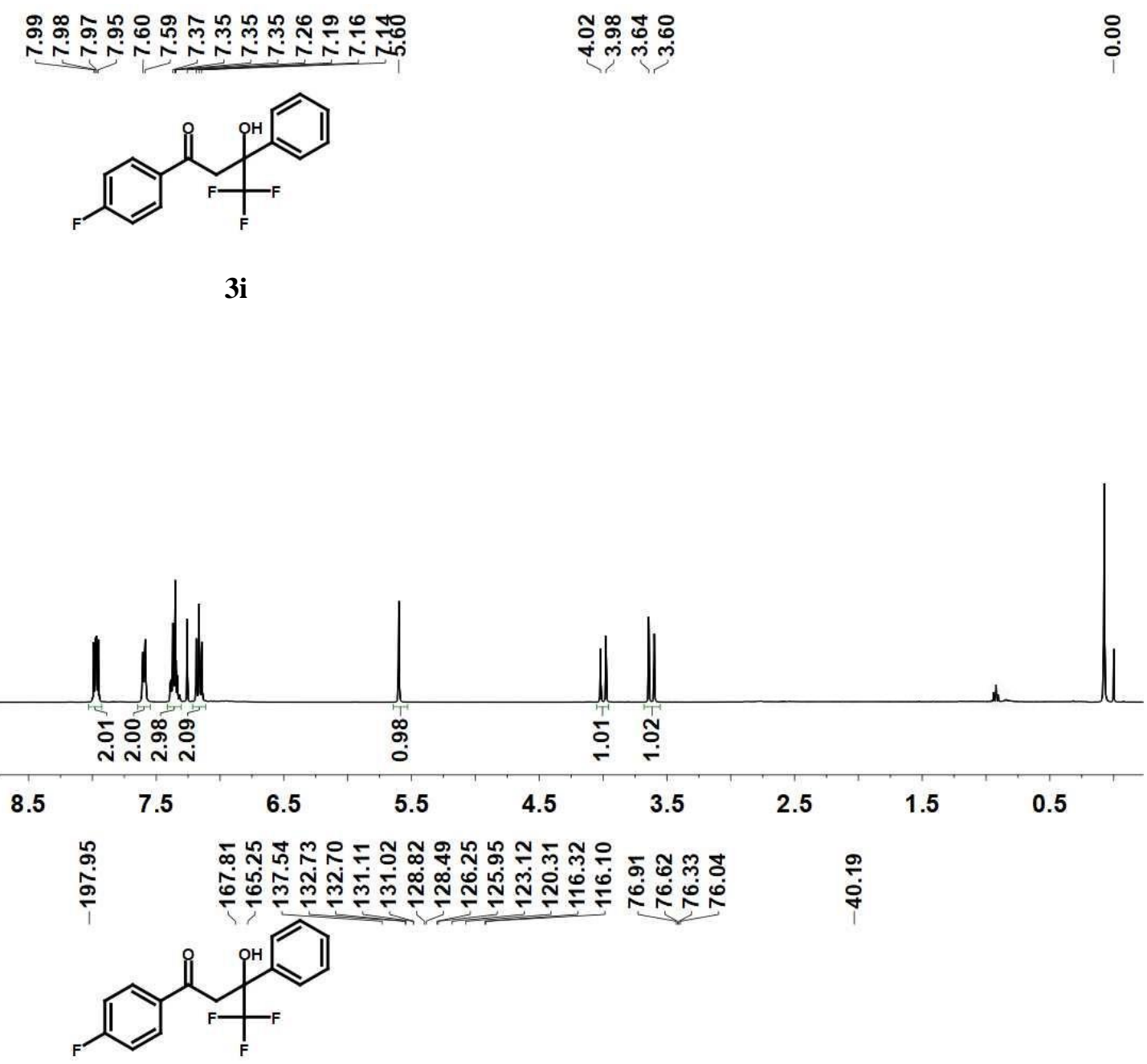

$3 i$

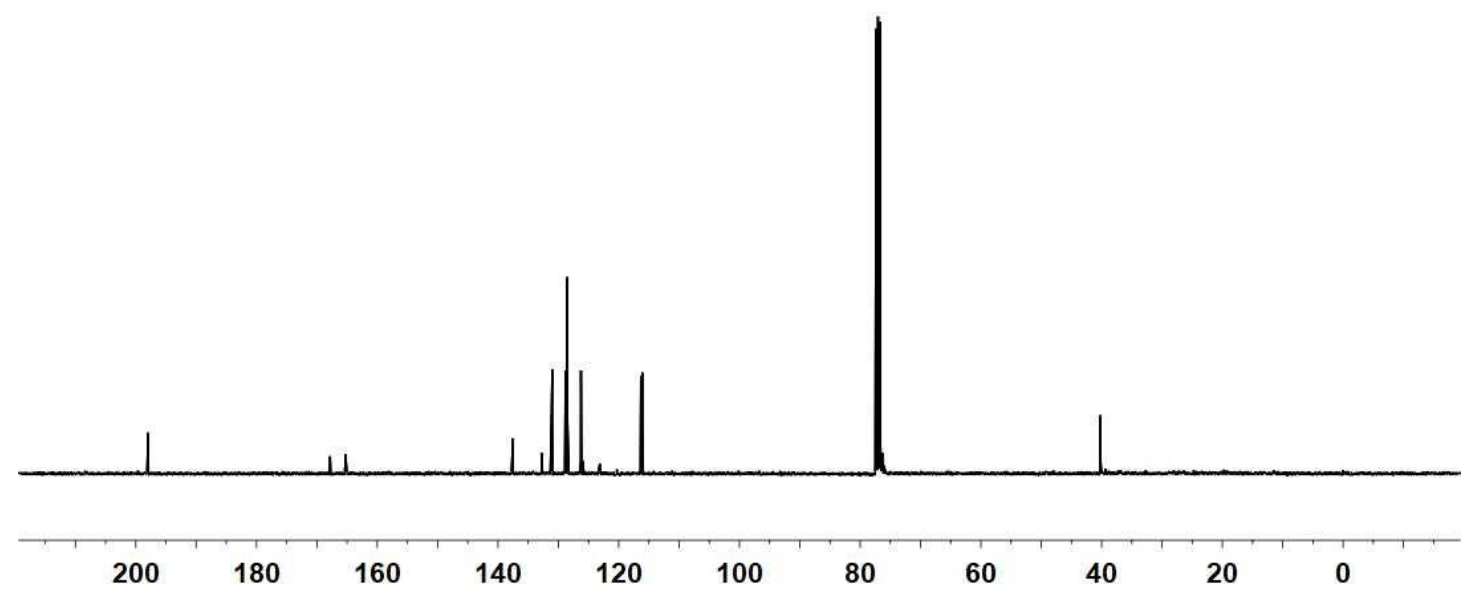


<smiles>O=C(CC(O)(c1ccccc1)C(F)(F)F)c1ccc(F)cc1</smiles>

$3 \mathbf{i}$

กั

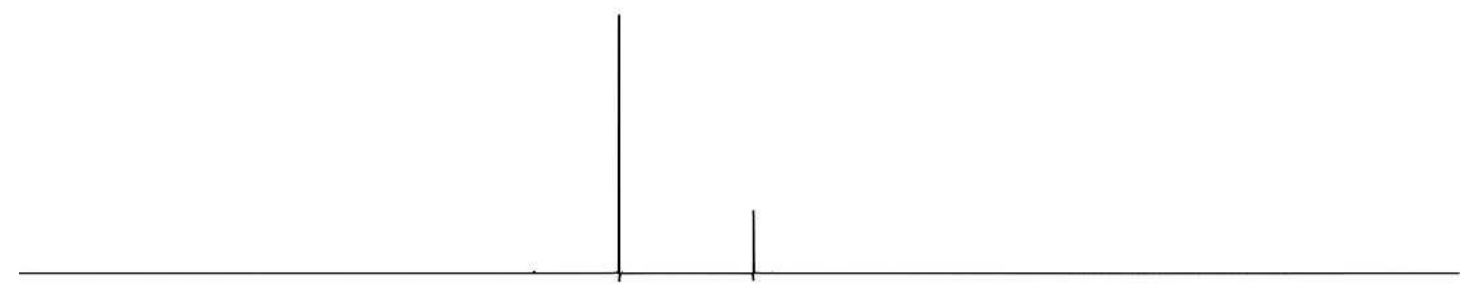

\begin{tabular}{llllllllllll}
\hline & -10 & -30 & -50 & -70 & -90 & -110 & -130 & -150 & -170 & -190 & -210
\end{tabular}

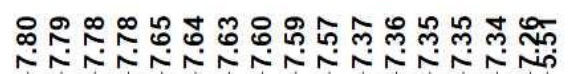

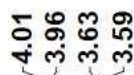

$\stackrel{8}{i}$<smiles>O=C(CC(O)(c1ccccc1)C(F)(F)F)c1ccc(Br)cc1</smiles>

3j

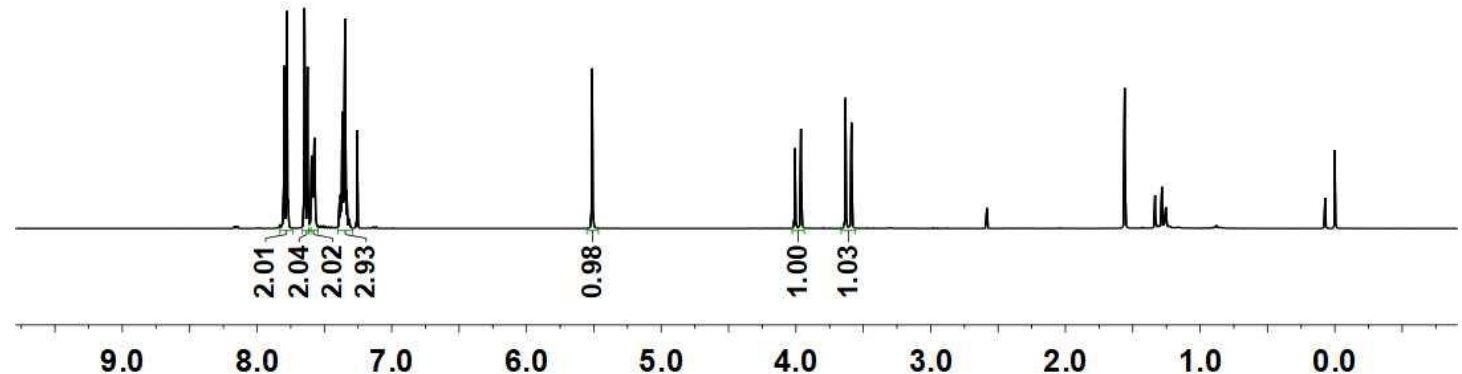




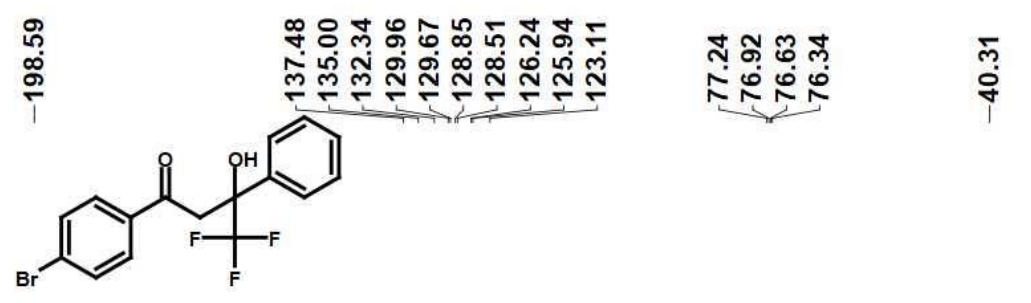

3j

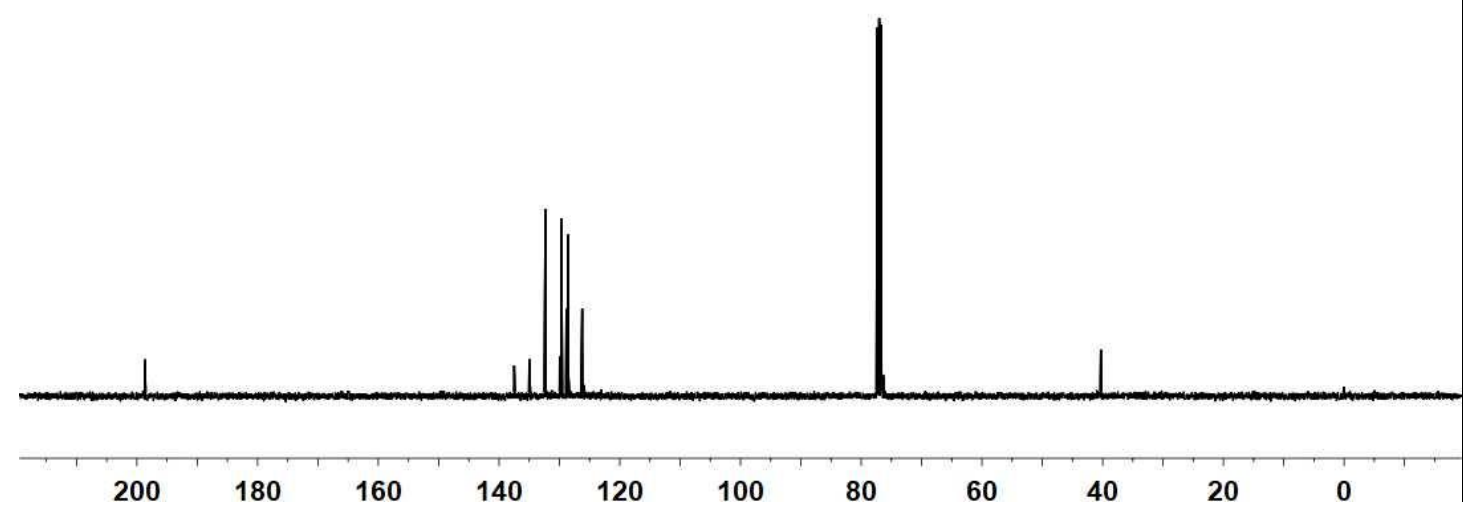

$\stackrel{\infty}{\stackrel{\infty}{\infty}}$<smiles>O=C(CC(O)(c1ccccc1)C(F)(F)F)c1ccc(Br)cc1</smiles> 
<smiles>O=C(CC(O)(c1ccccc1)C(F)(F)F)c1ccc(Cl)cc1</smiles>

3k
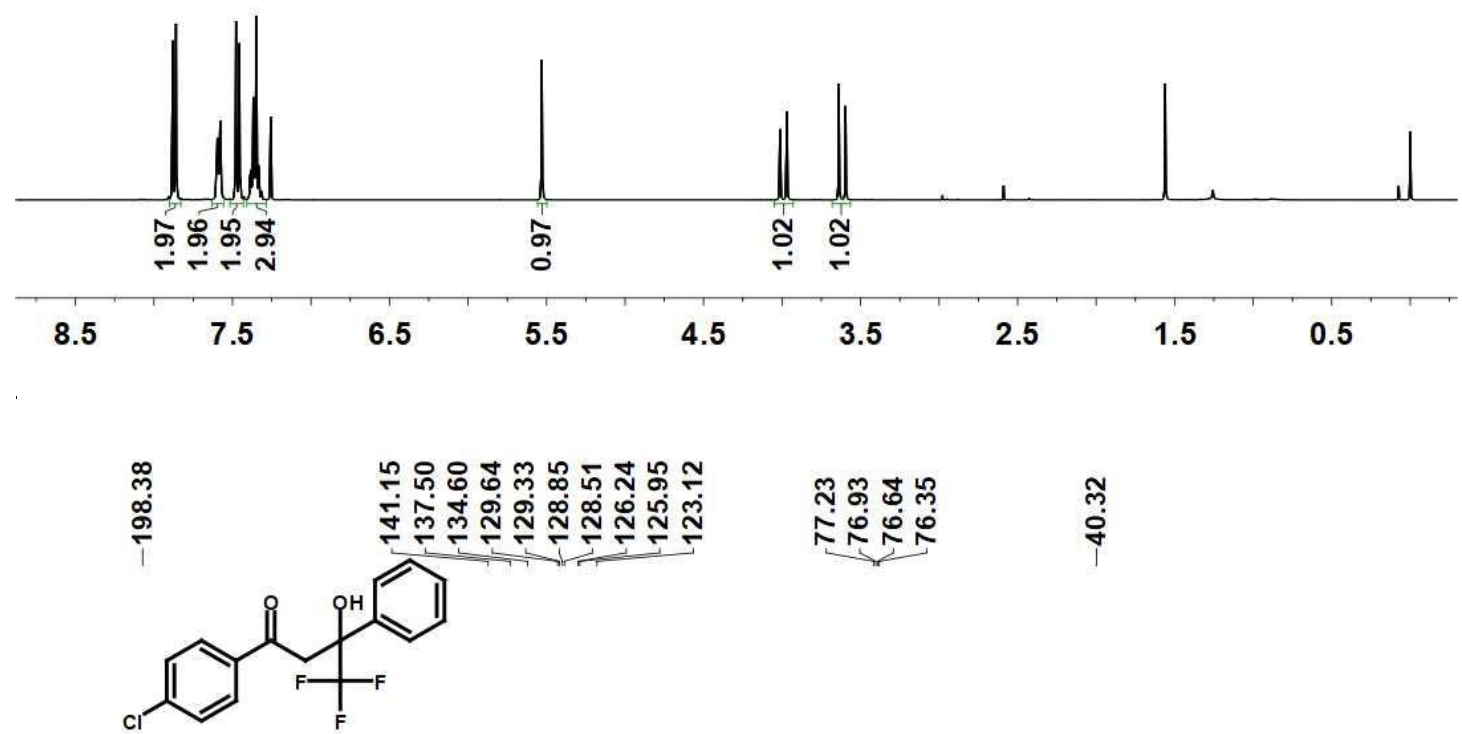

3k

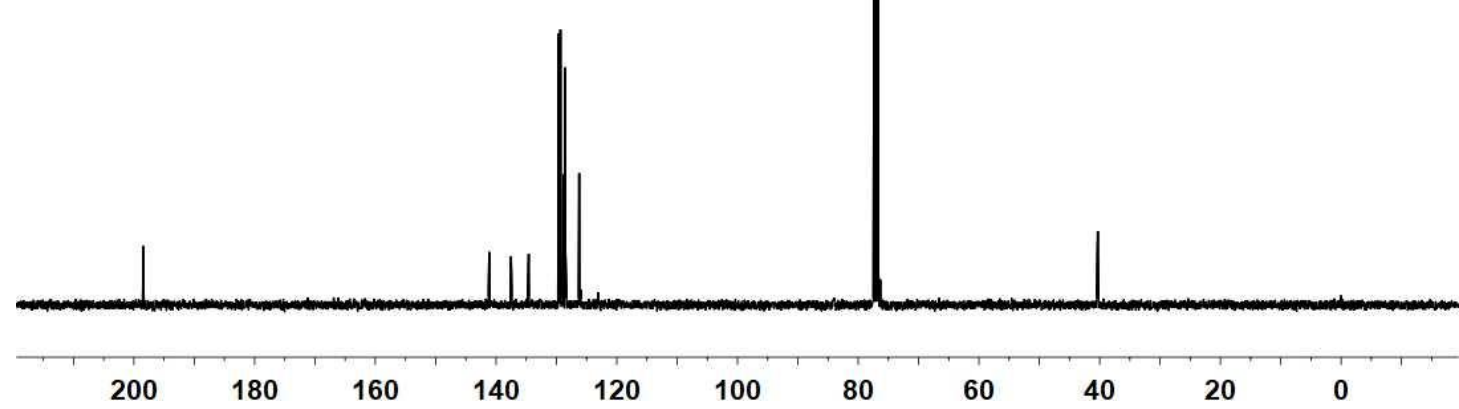




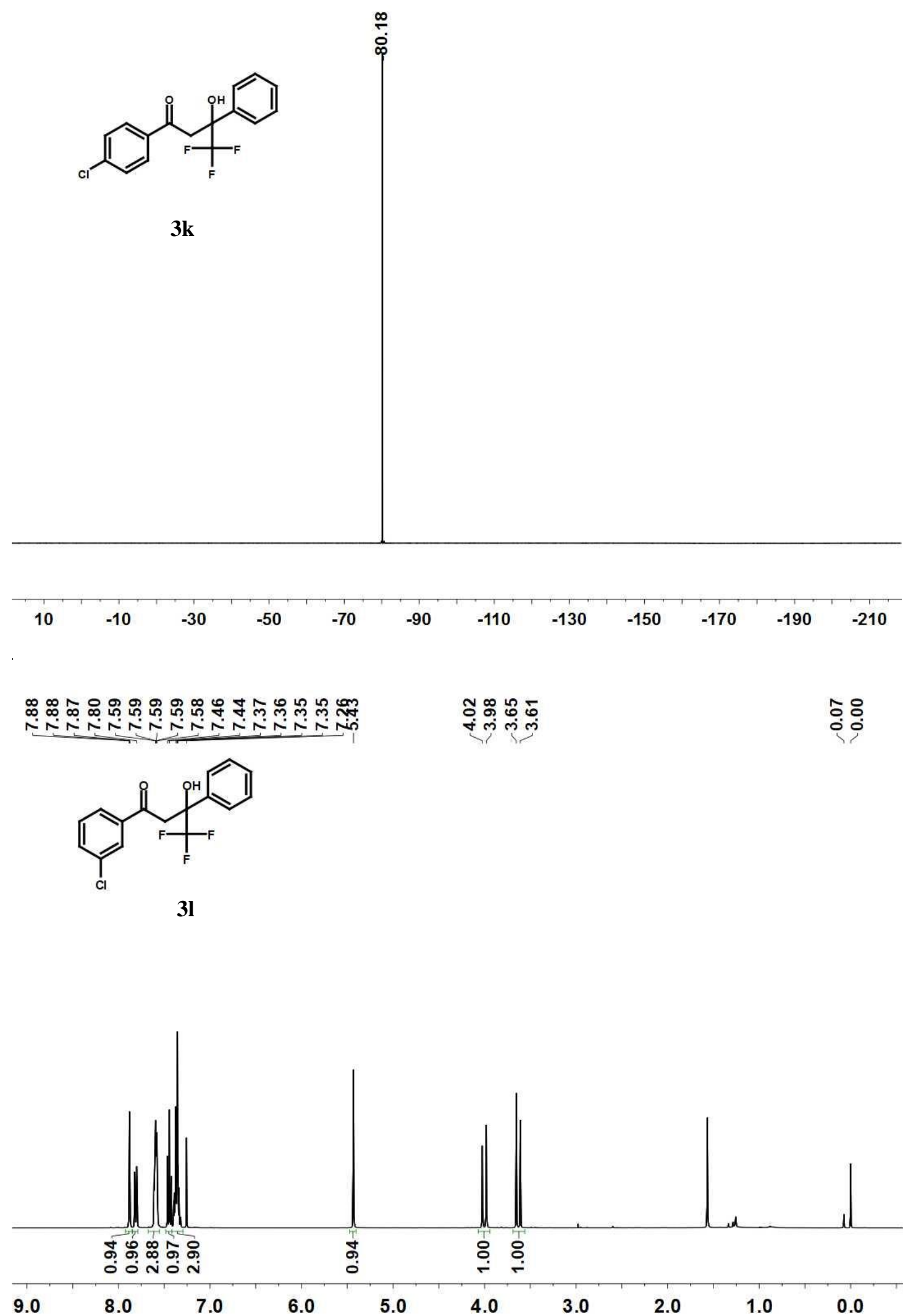




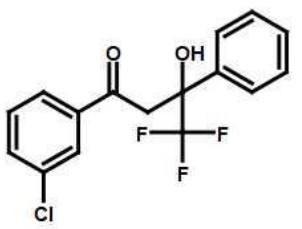

3I
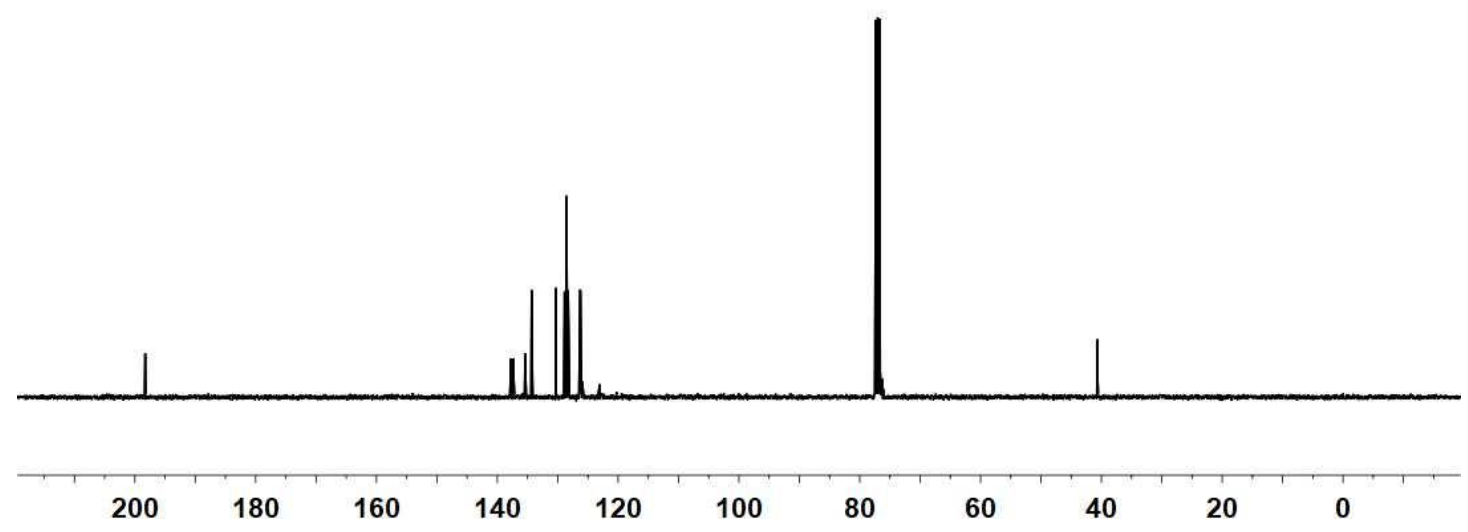

$80 \quad 60$

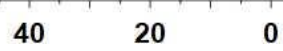

$\stackrel{9}{\stackrel{0}{\circ}}$

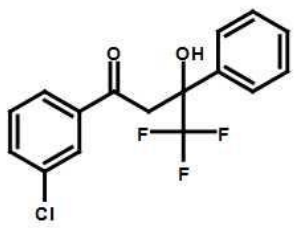

31 


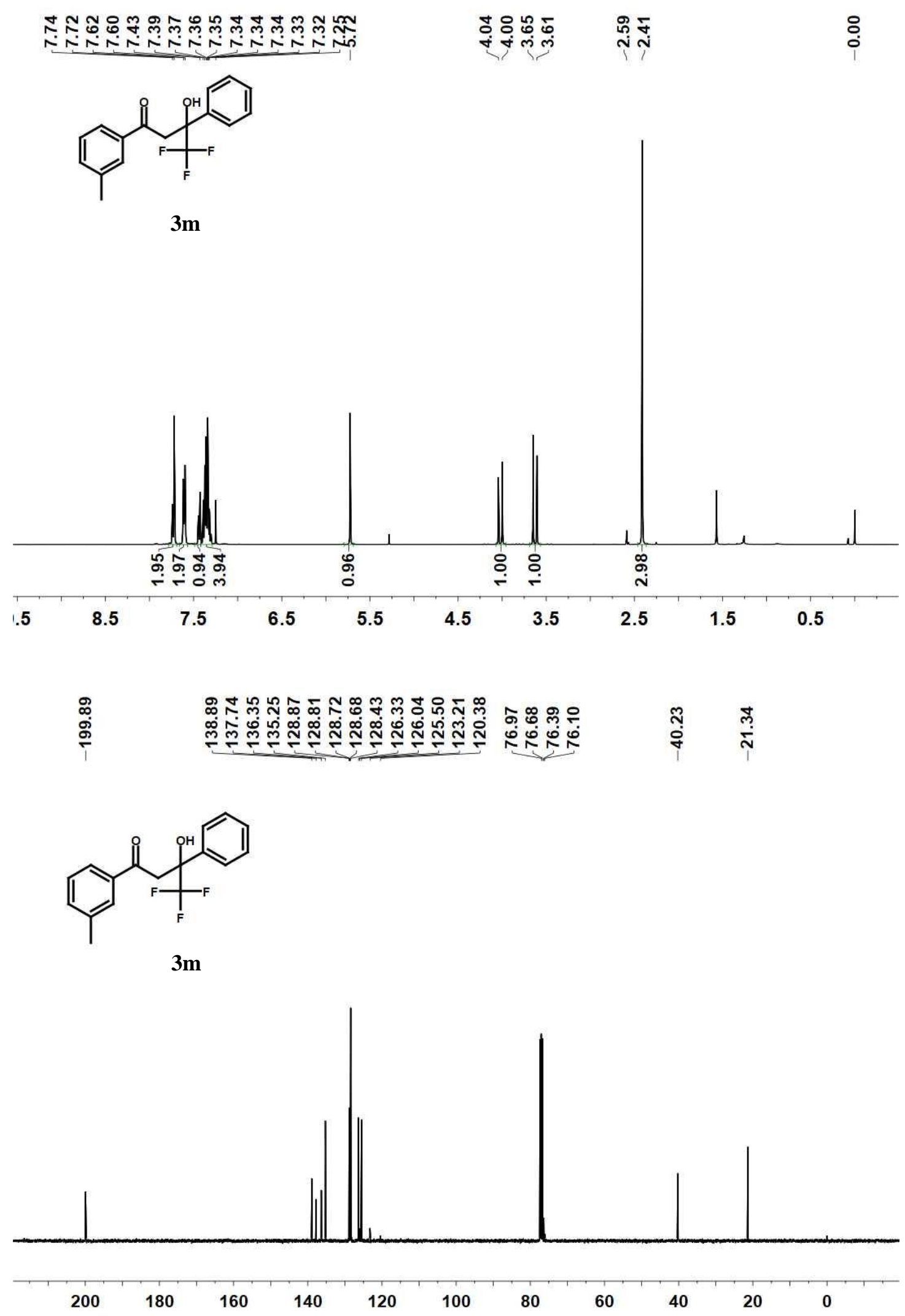




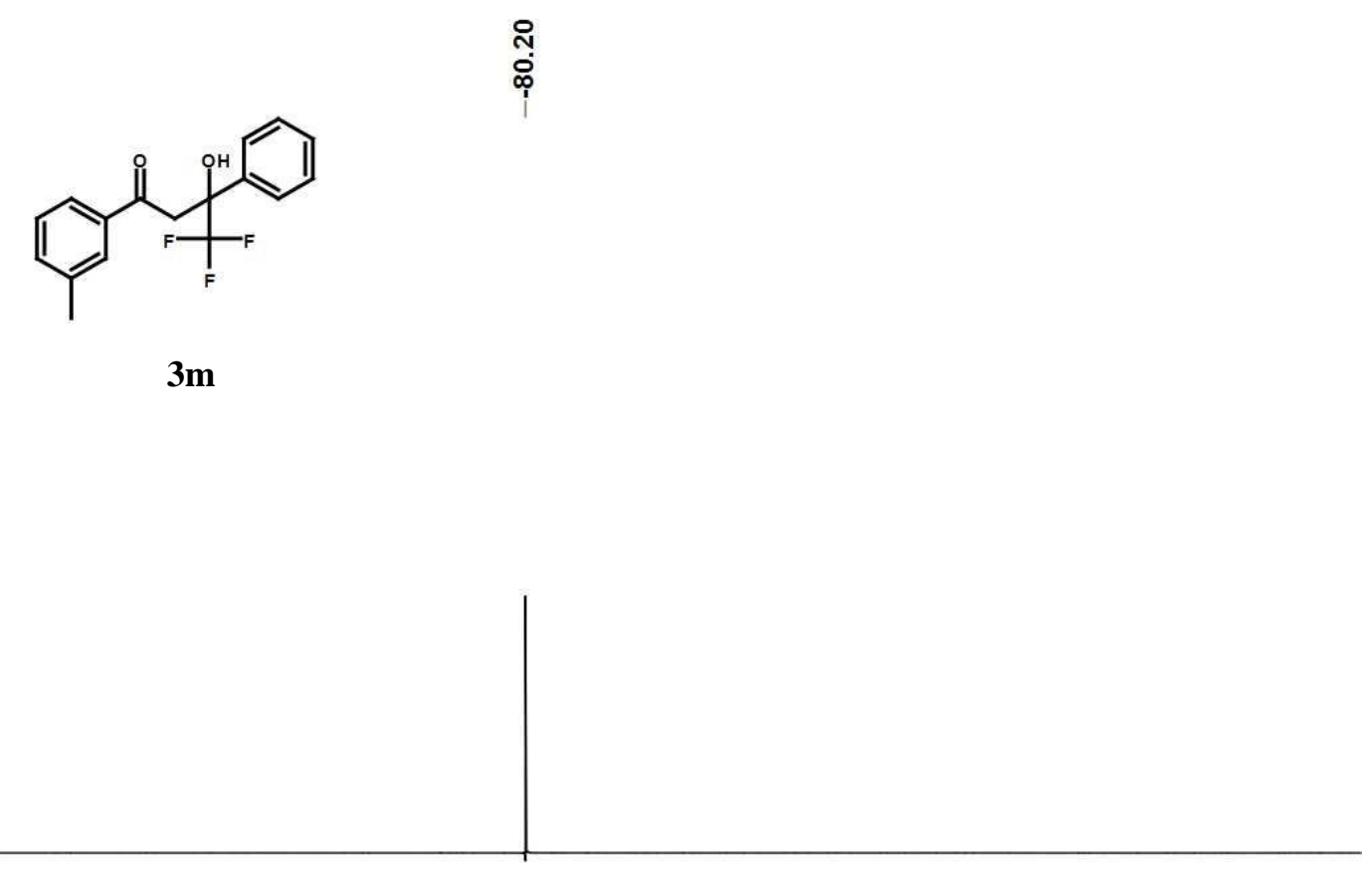

\begin{tabular}{llllllllllll}
\hline 10 & -10 & -30 & -50 & -70 & -90 & -110 & -130 & -150 & -170 & -190 & -210
\end{tabular}
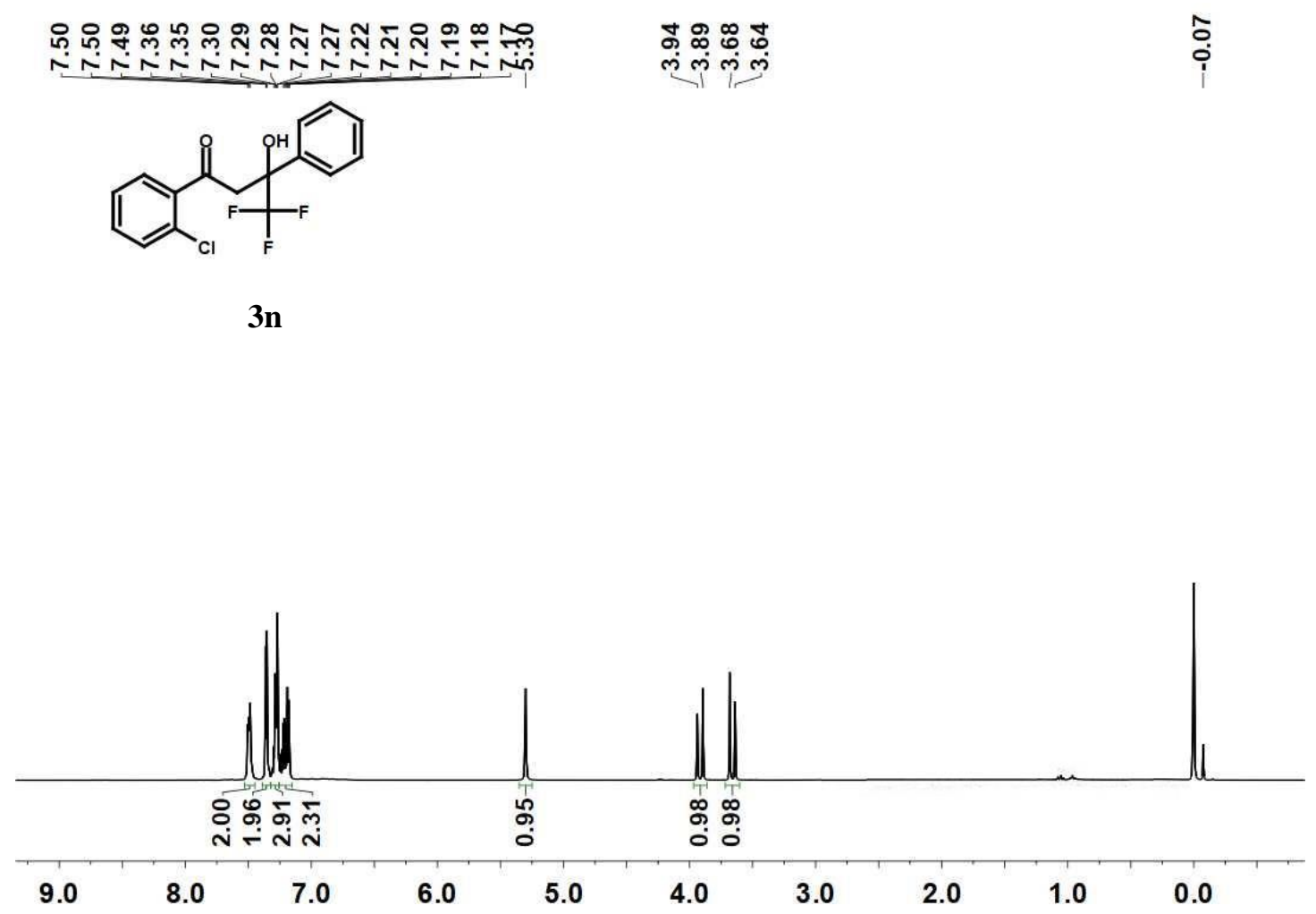


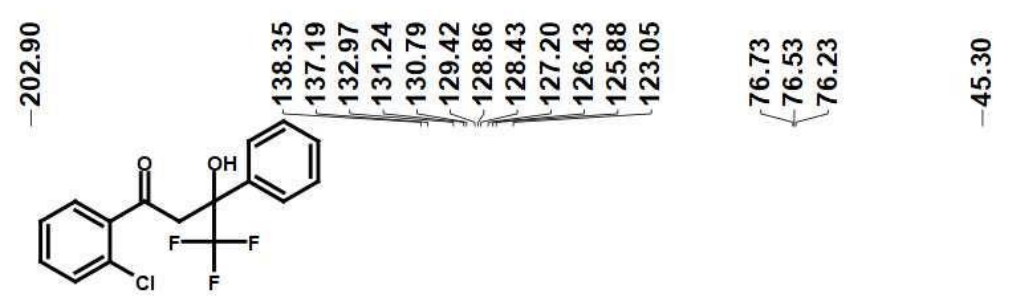

3n

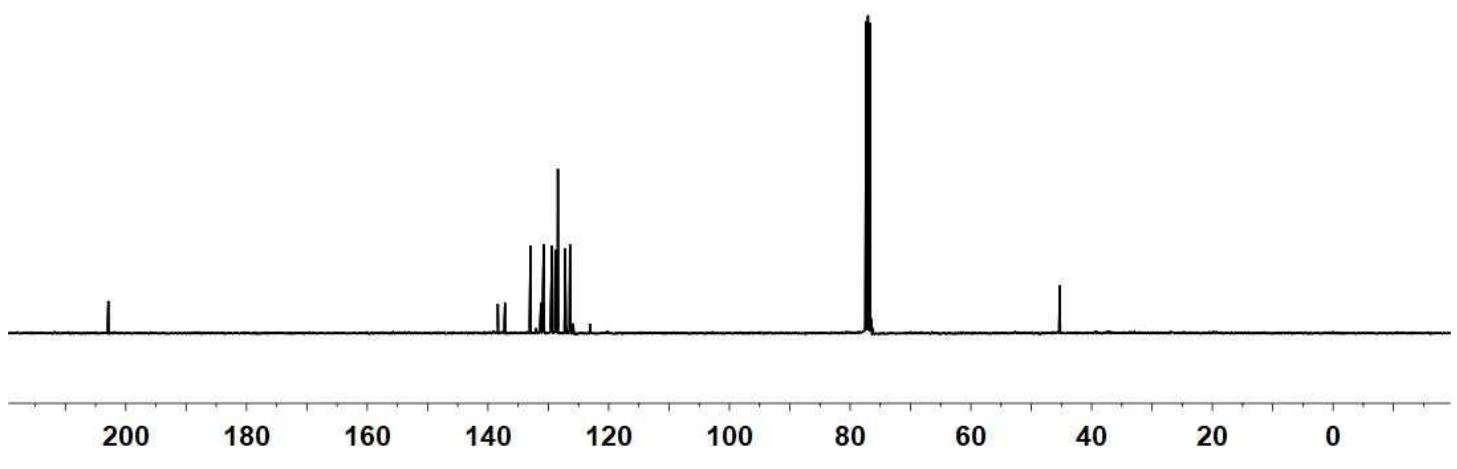

S37 


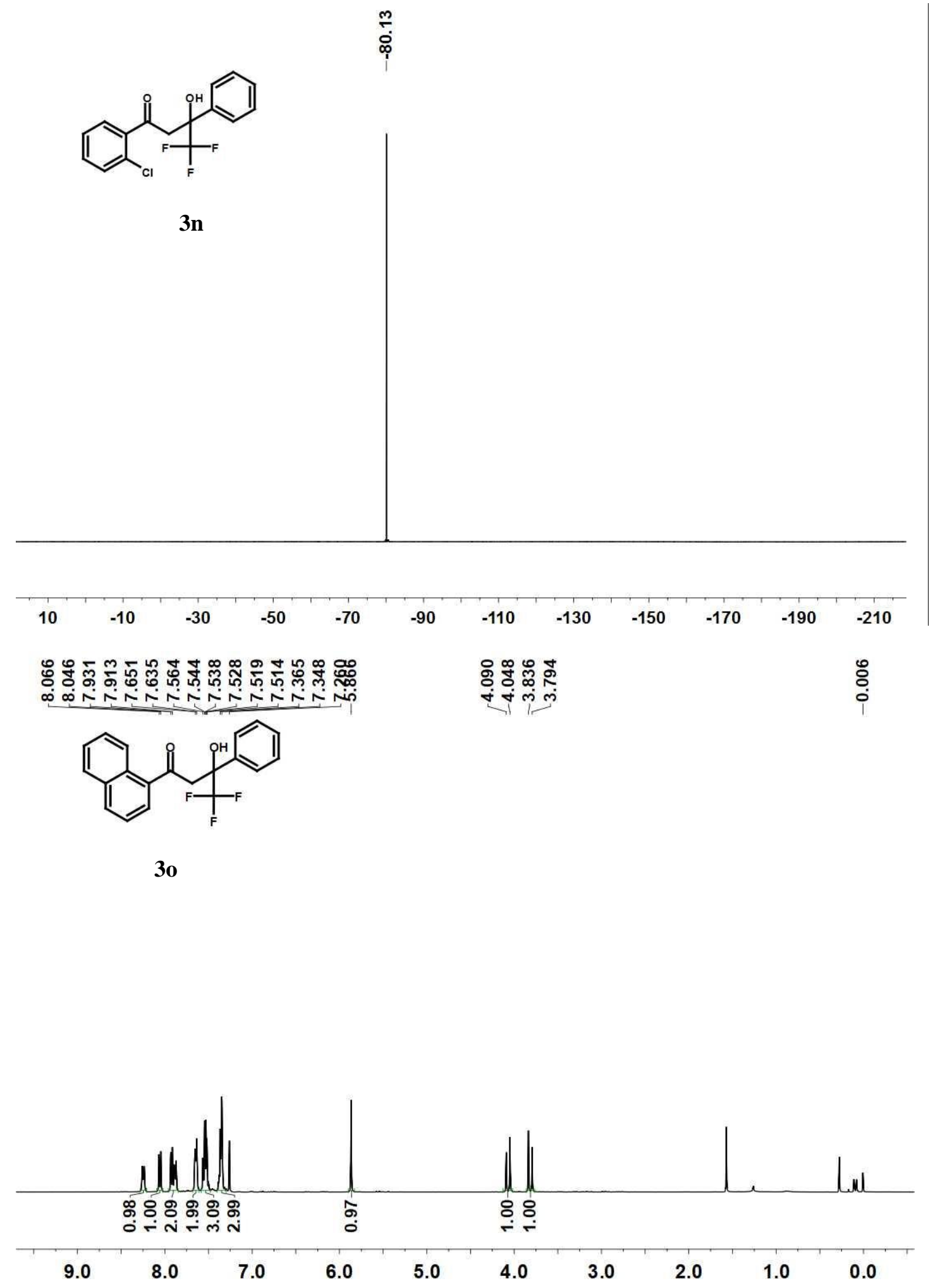




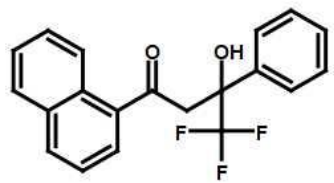

30

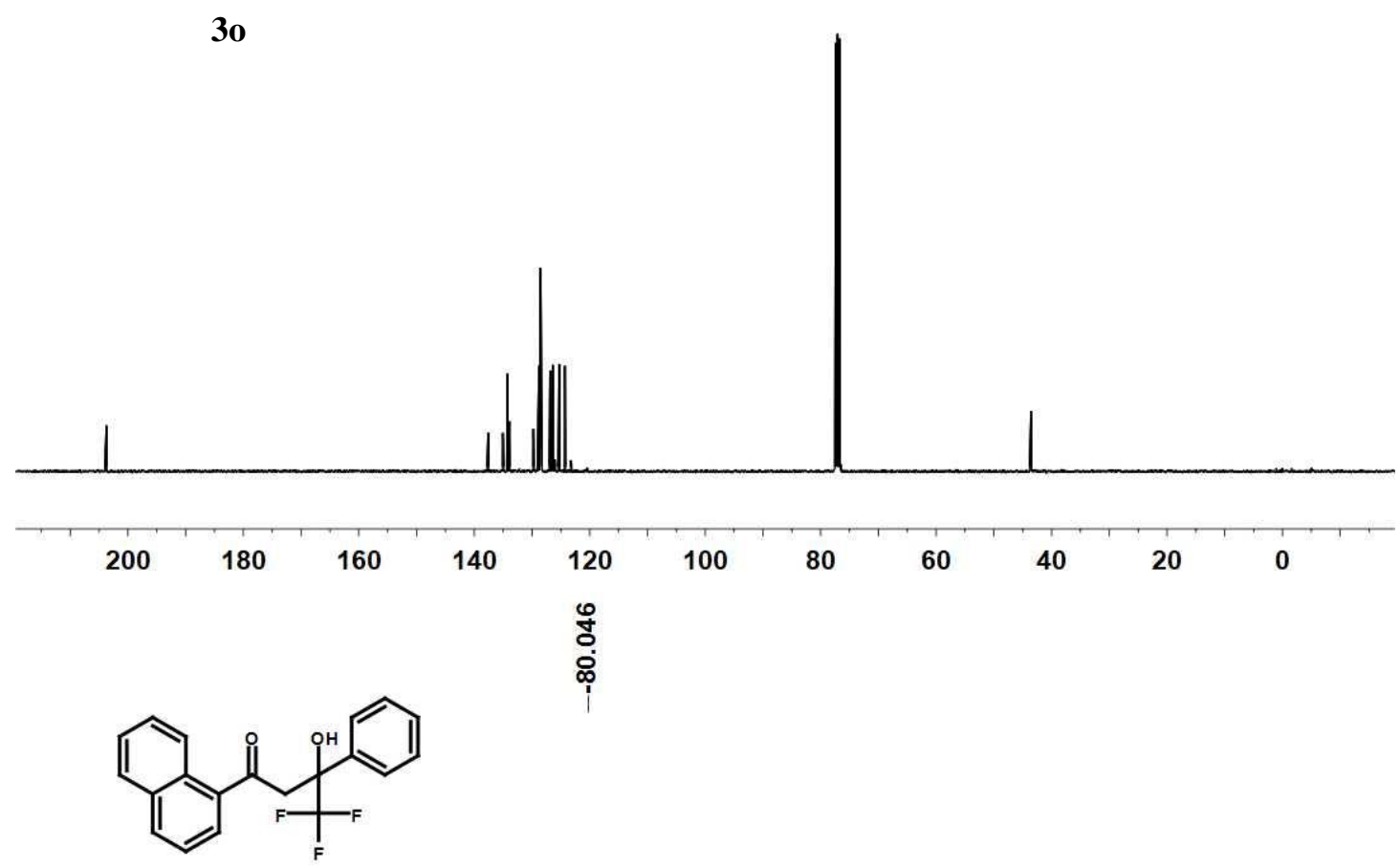

30

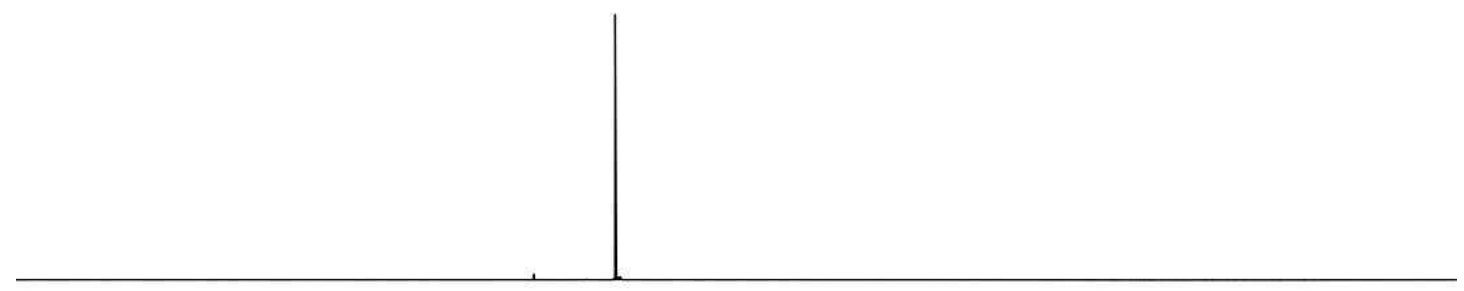

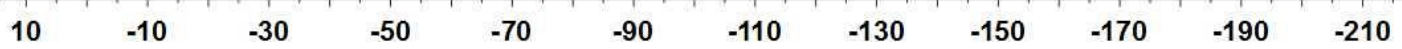




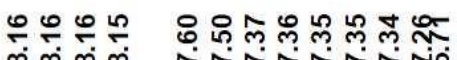

๑

웅<smiles>O=C(CC(O)(c1ccccc1)C(F)(F)F)c1ccsc1</smiles>

$3 p$

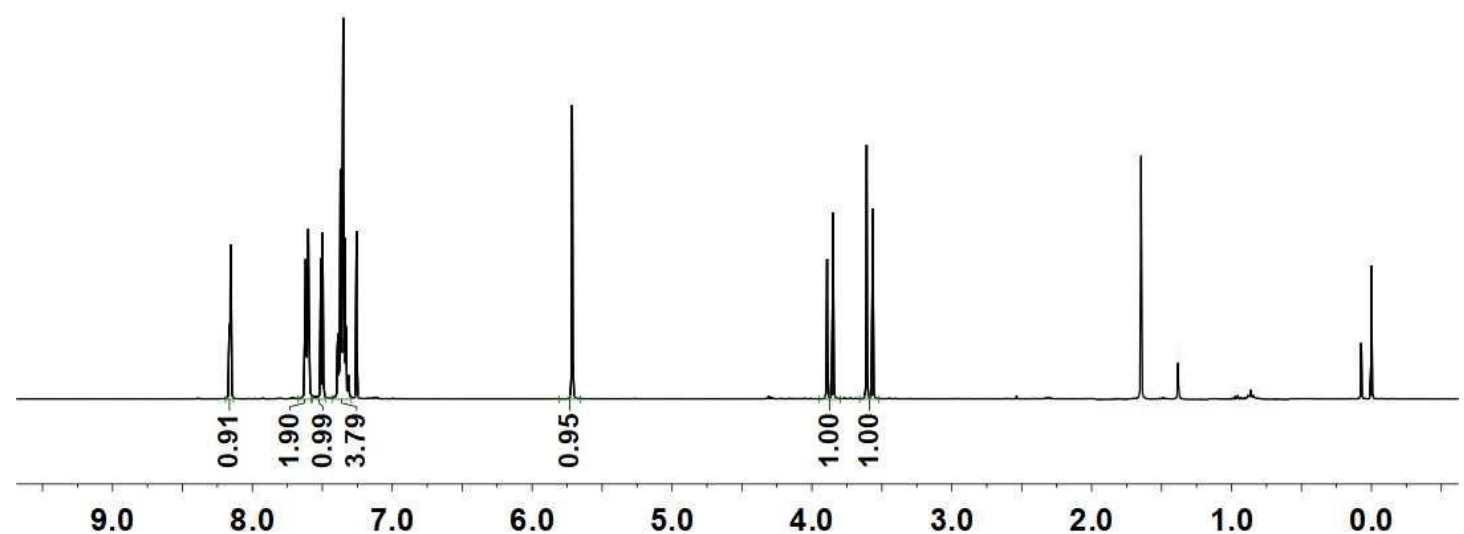

กั.

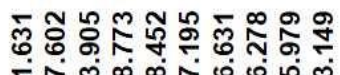

守岱

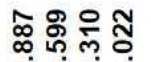

这通

ஜீ<smiles>O=C(CC(O)(c1ccccc1)C(F)(F)F)c1ccsc1</smiles>

$3 p$

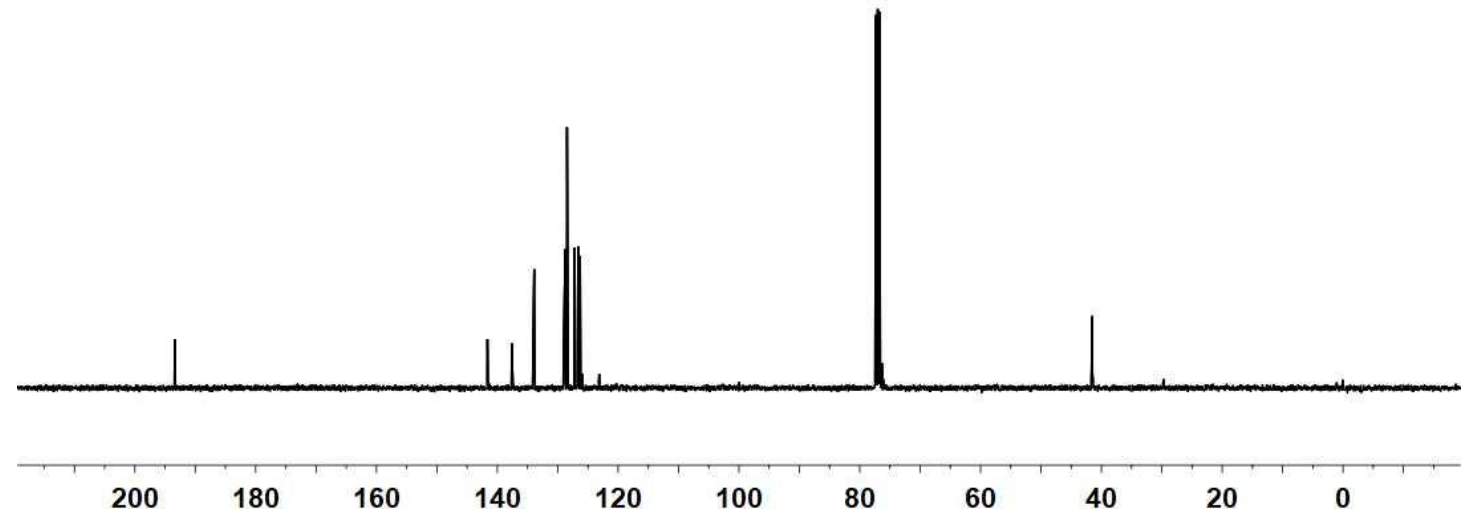



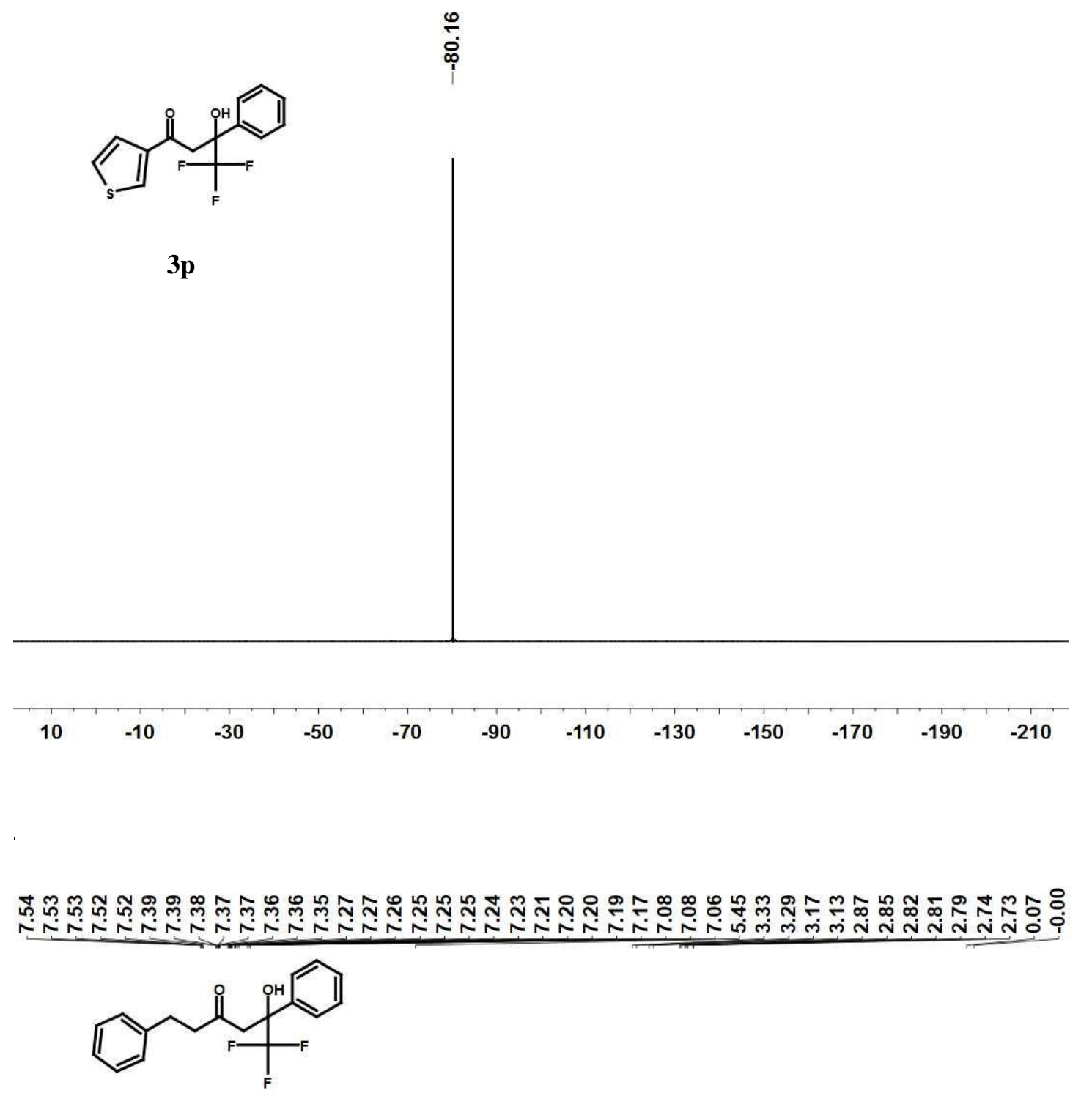

$3 q$

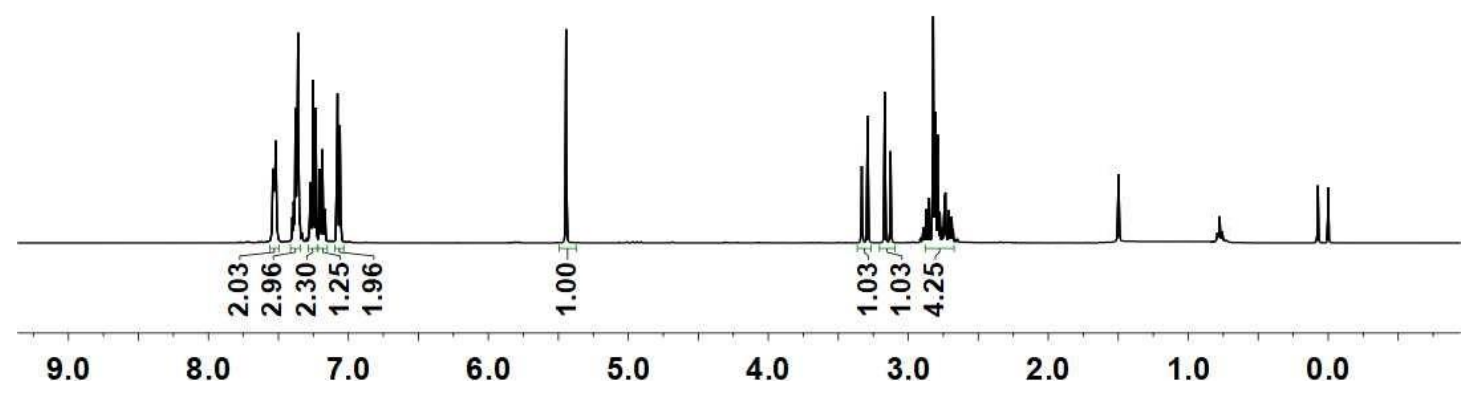


<smiles>O=C(CCc1ccccc1)CC(O)(c1ccccc1)C(F)(F)F</smiles>

$3 \mathbf{q}$

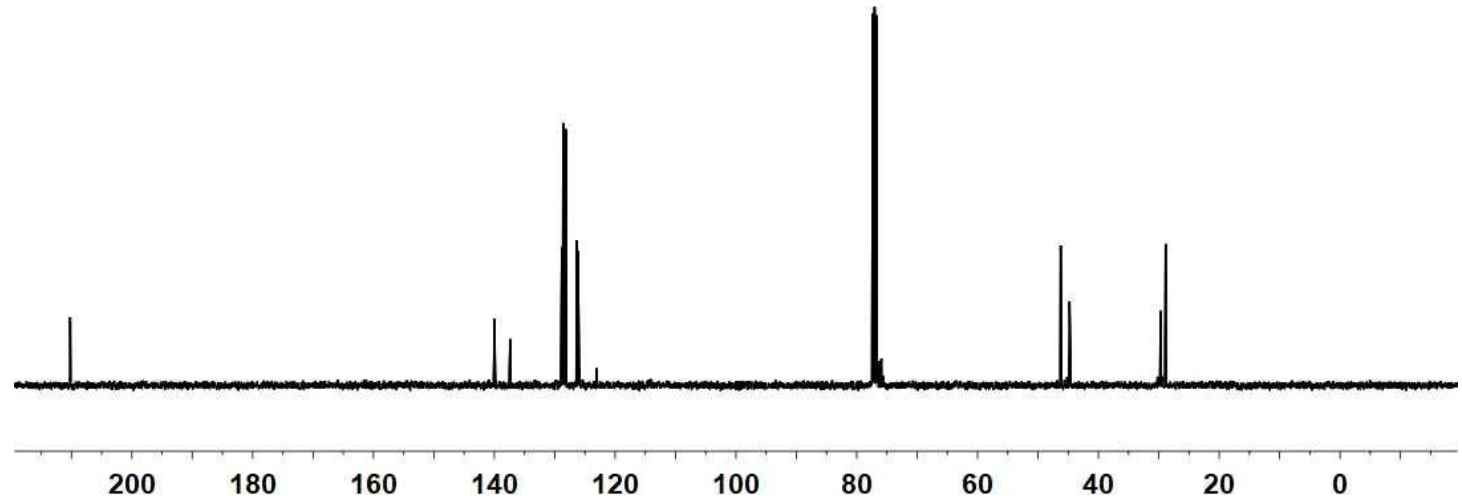

స̃.<smiles>O=C(CCc1ccccc1)CC(O)(c1ccccc1)C(F)(F)F</smiles>

$3 \mathbf{q}$

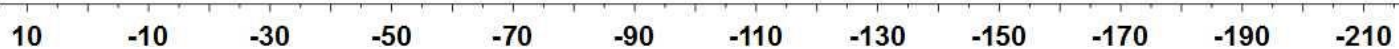




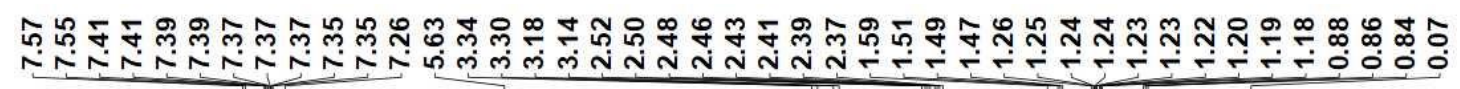

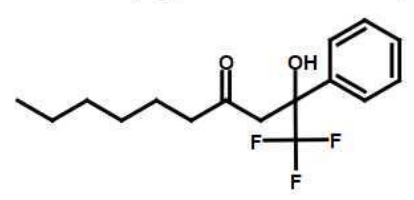

$3 r$

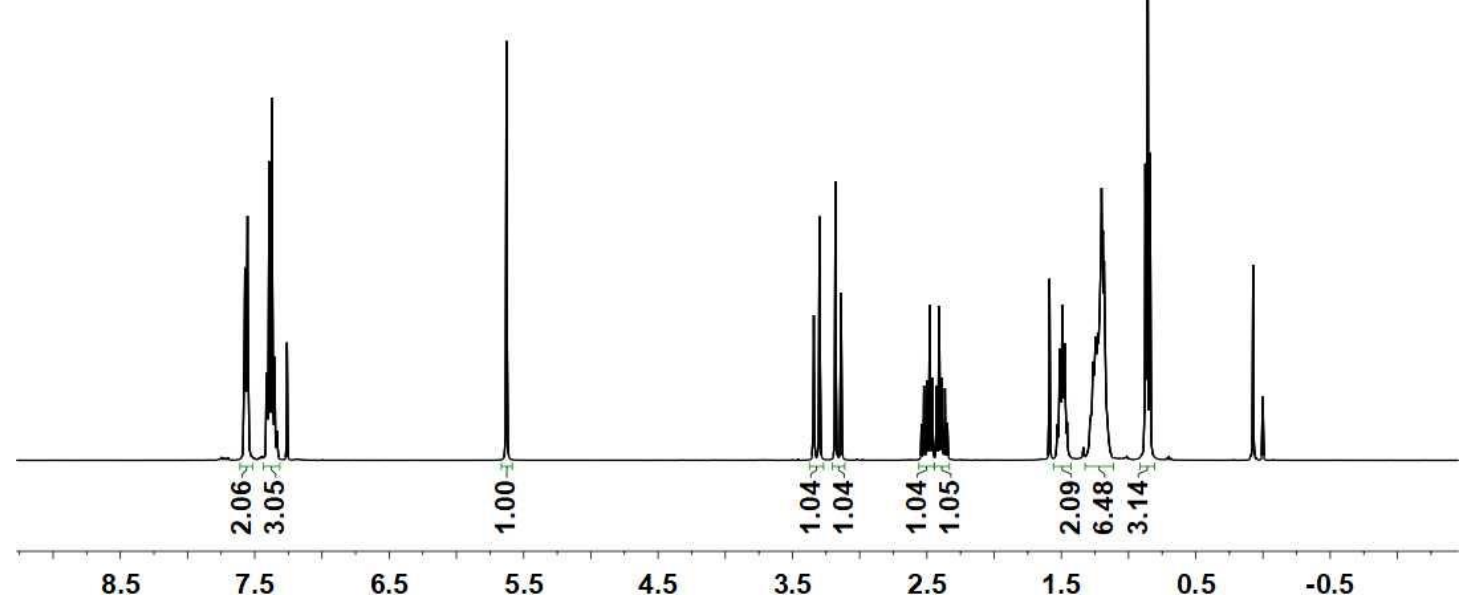

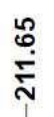

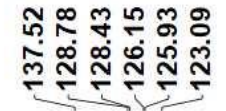

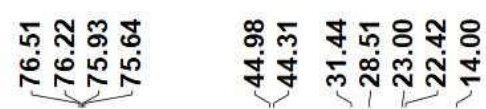

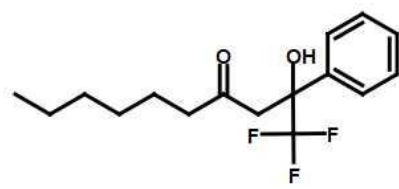

$3 r$

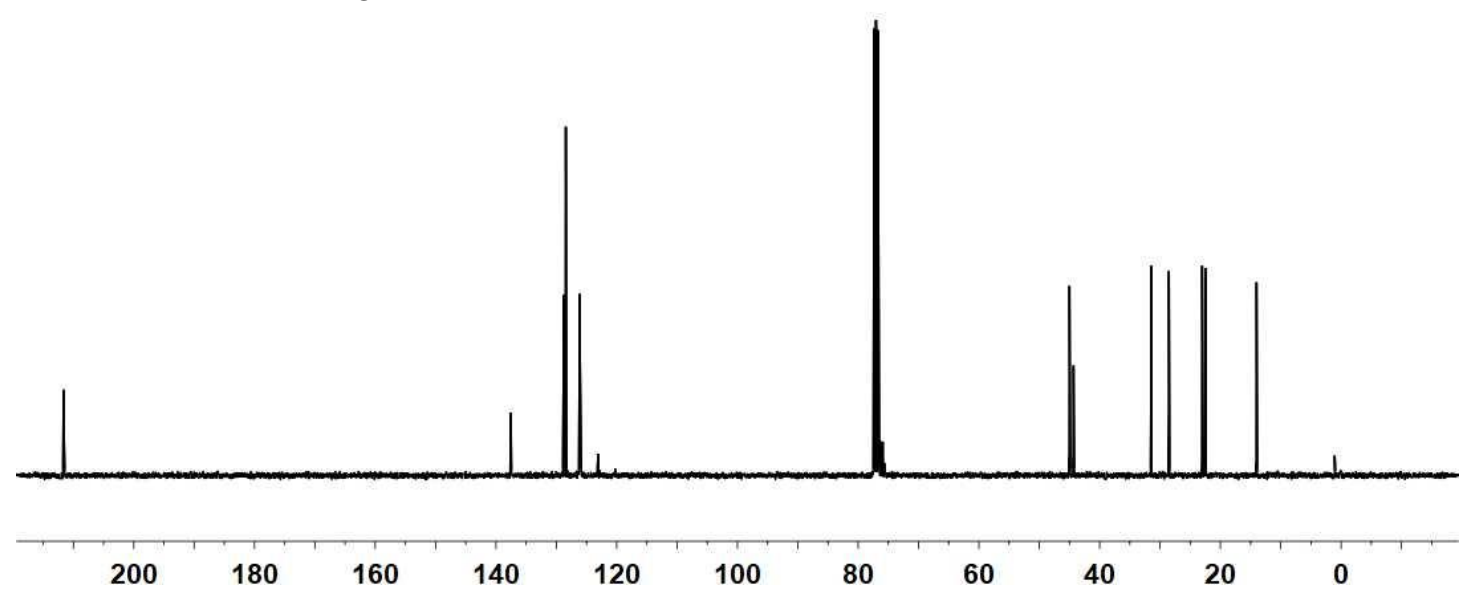




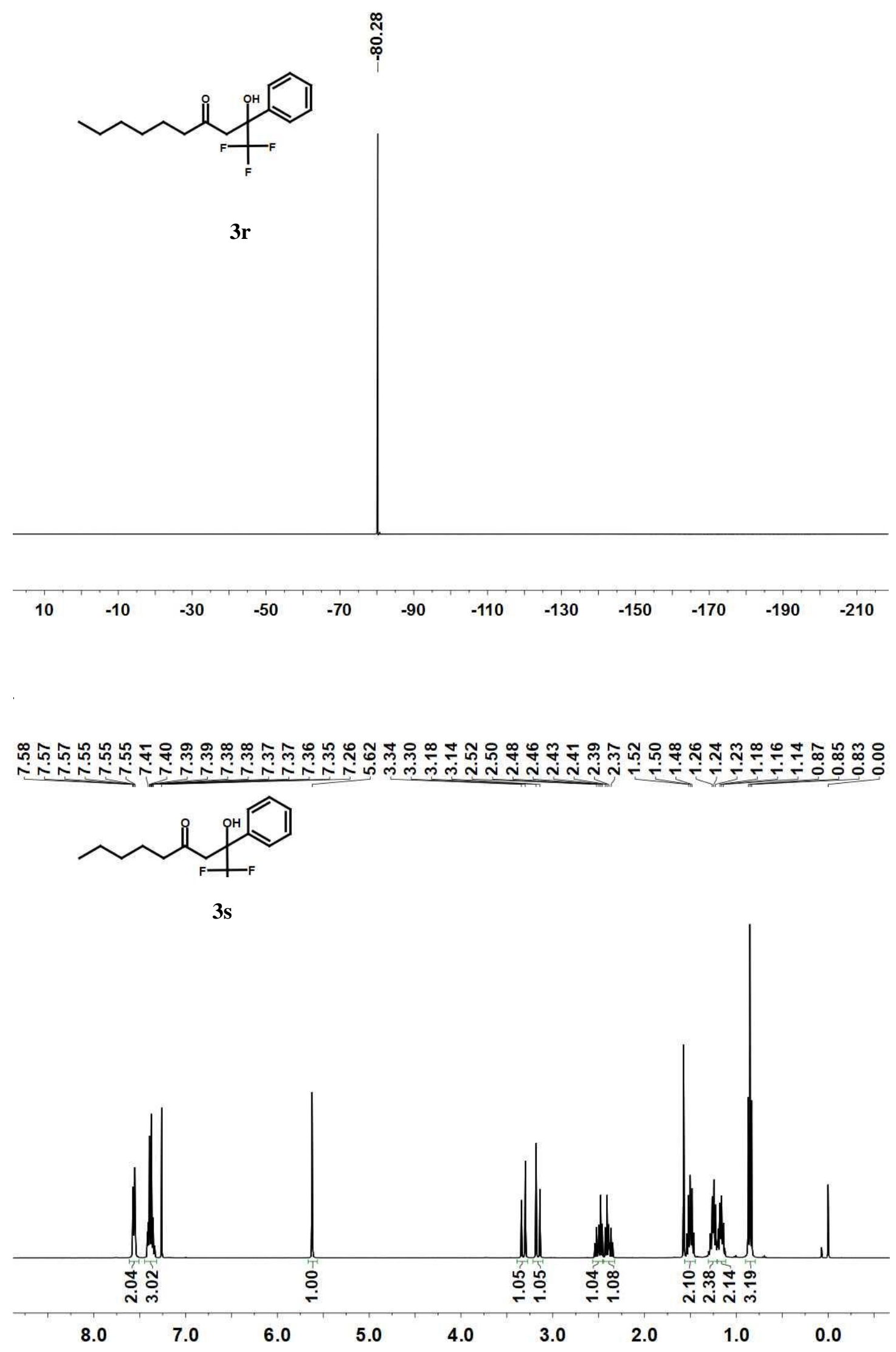



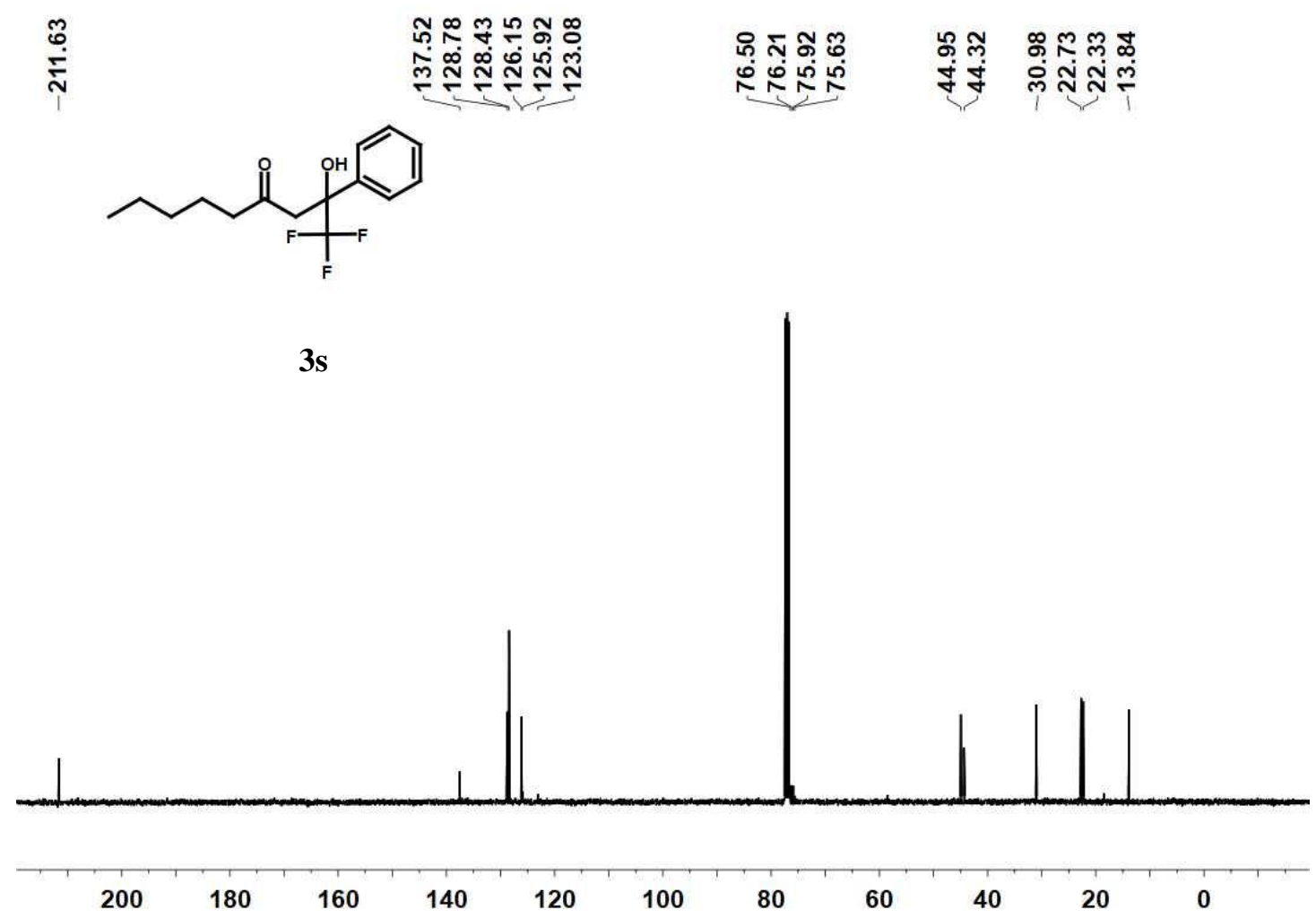

$\stackrel{\substack{\infty \\ \hdashline}}{\infty}$

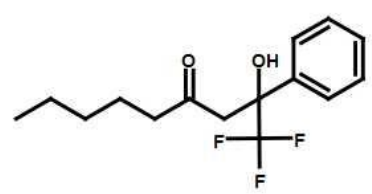

$3 s$

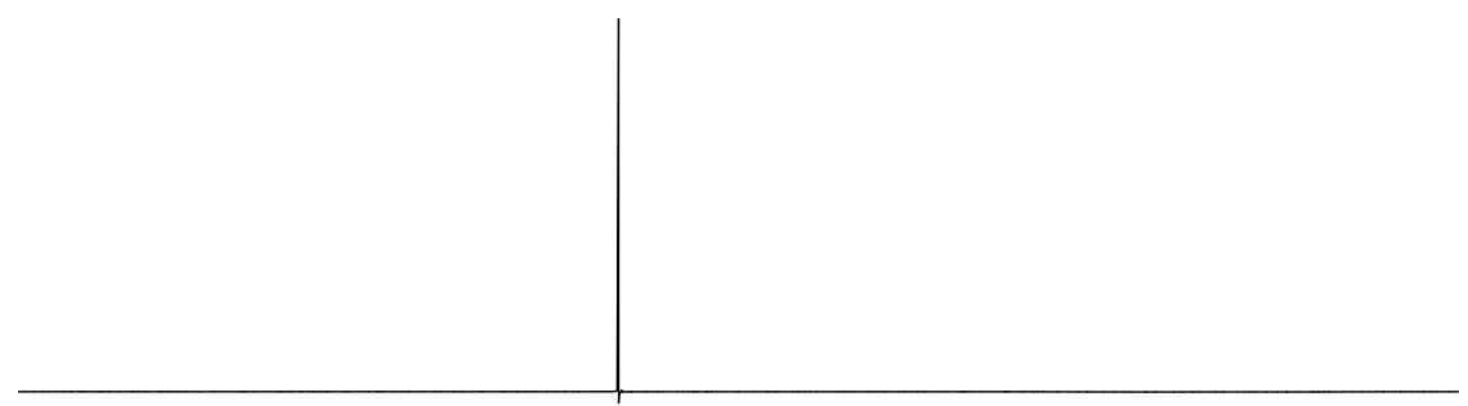

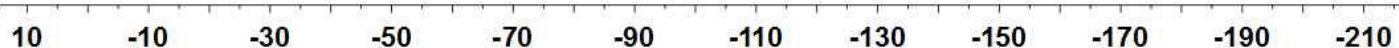




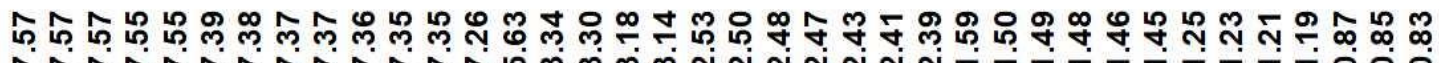

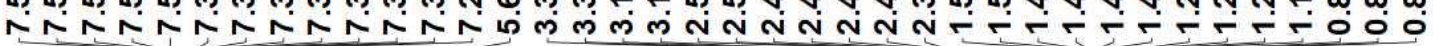

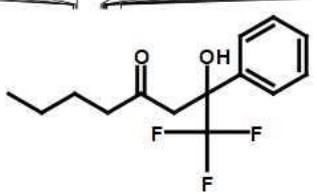

$3 \mathbf{t}$
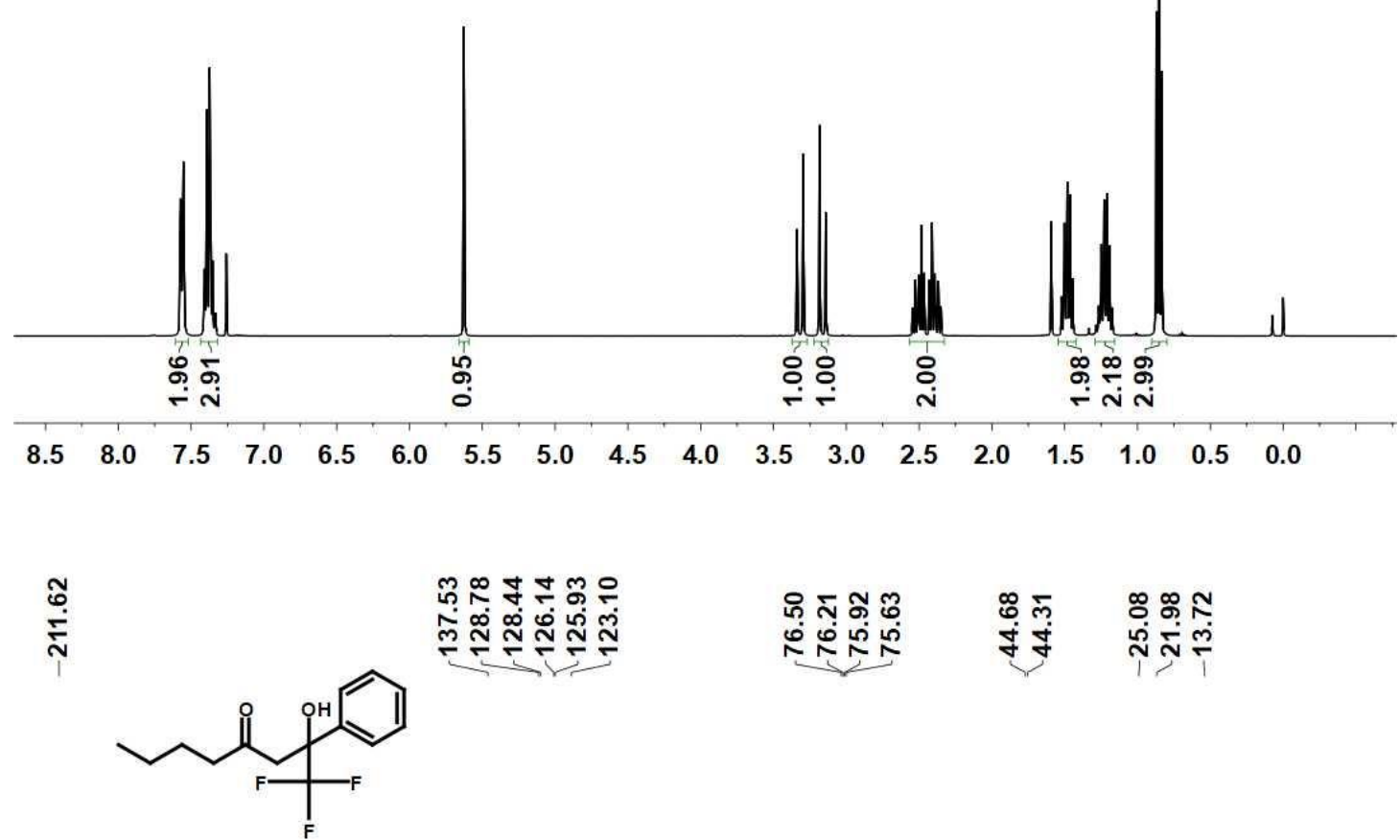

$3 \mathbf{t}$

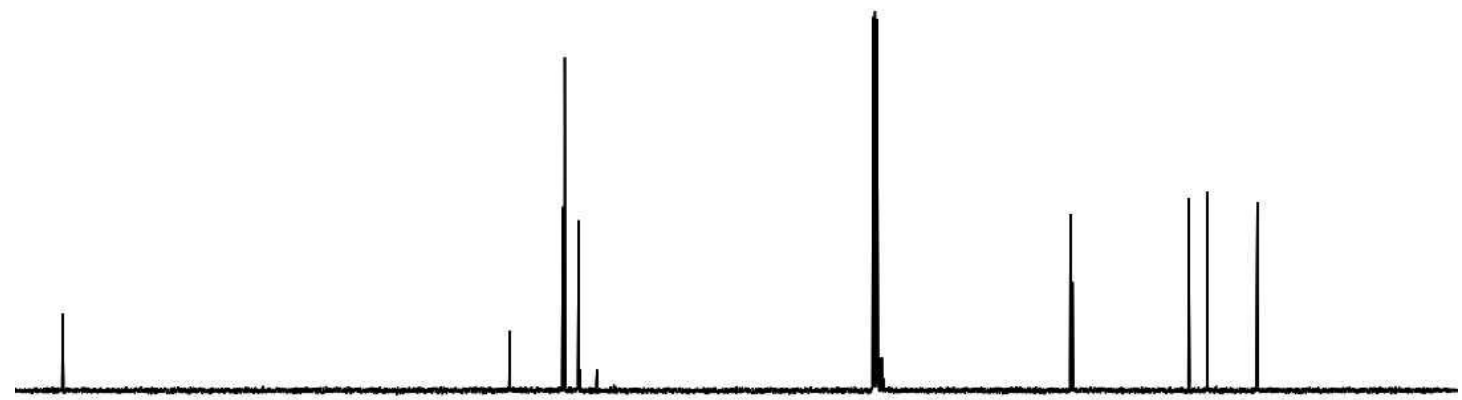

200

180

160

140

120

100

80

60

$40 \quad 20 \quad 0$ 


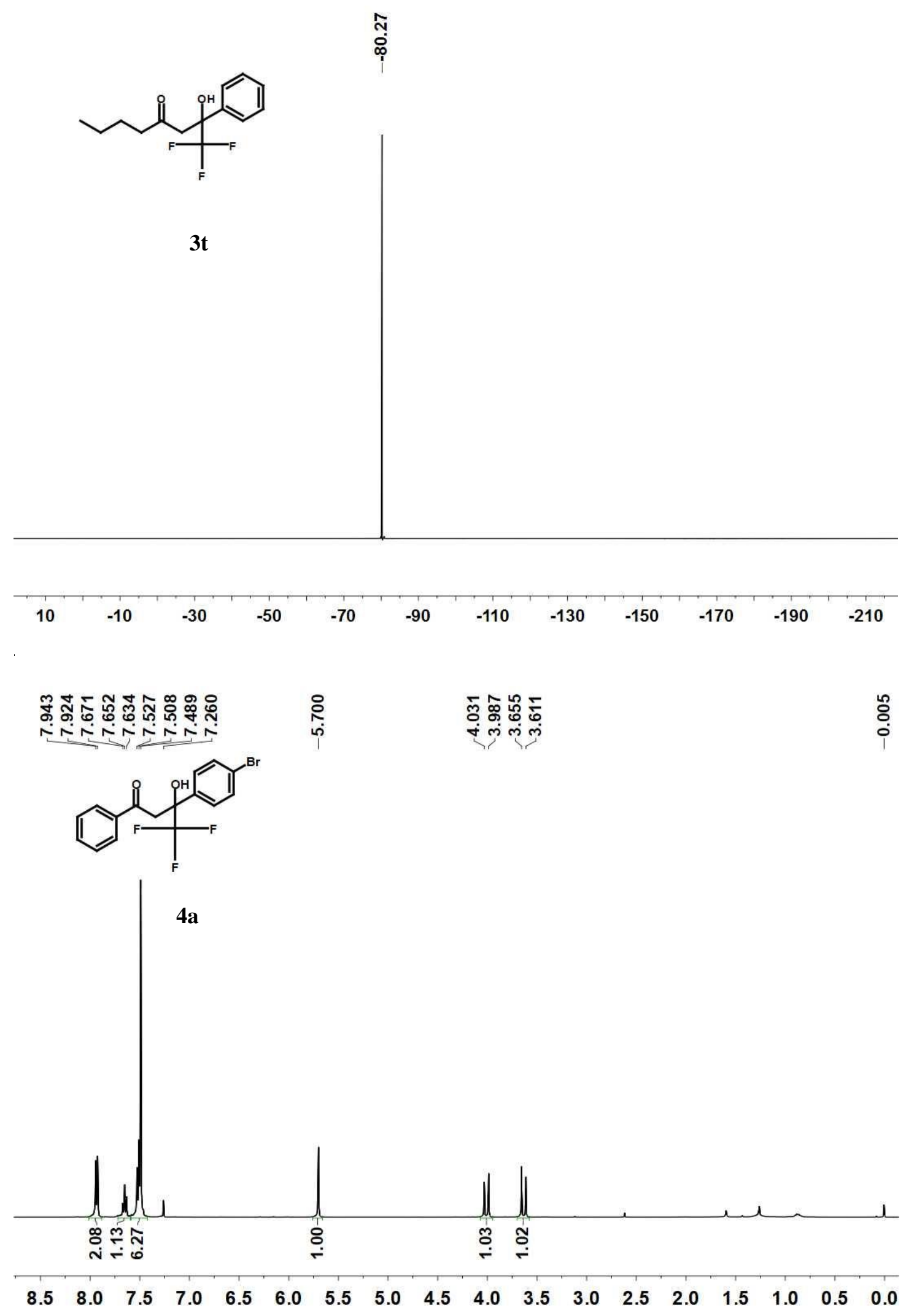


충 웅용

它过它

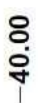

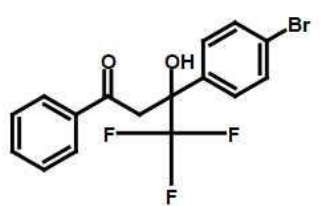

$4 a$

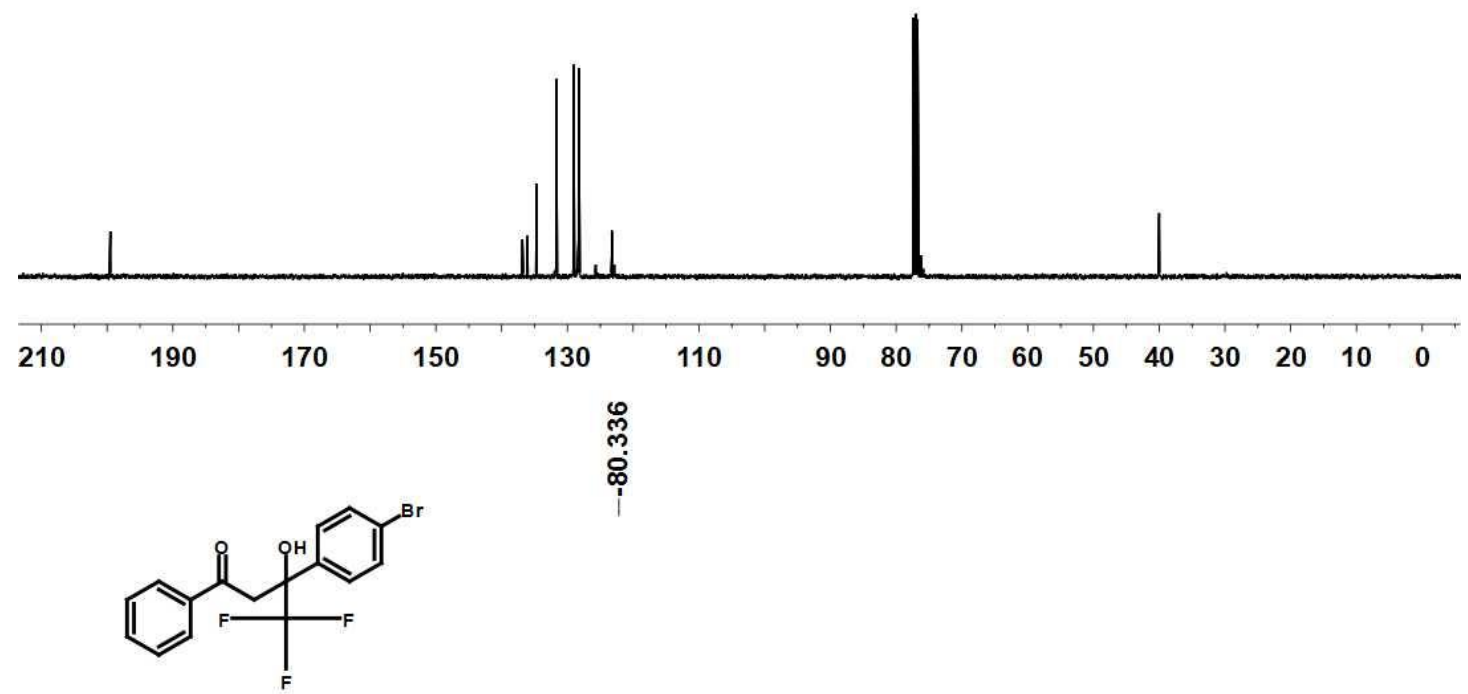

$4 a$

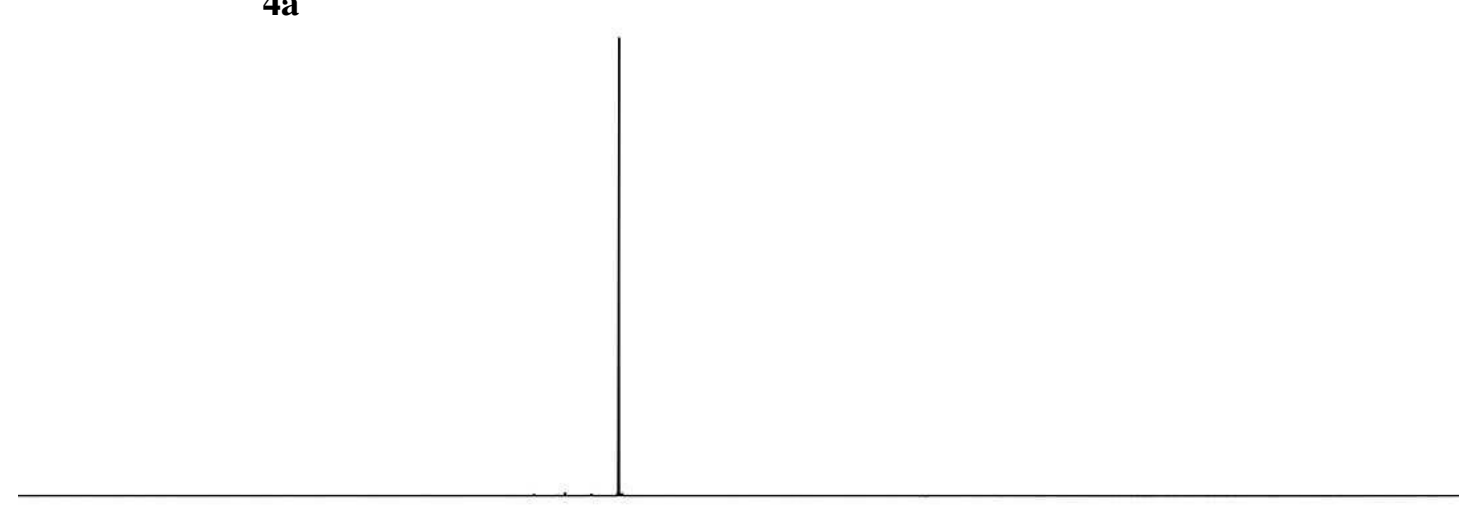

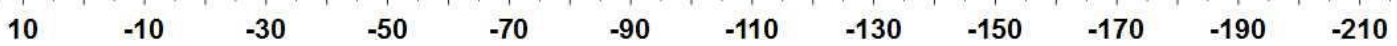




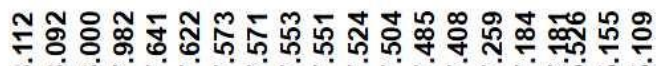

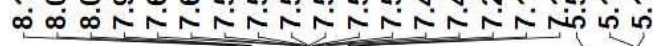

结 :

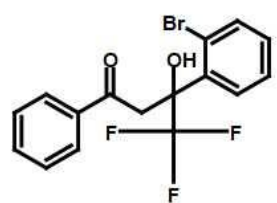

4b
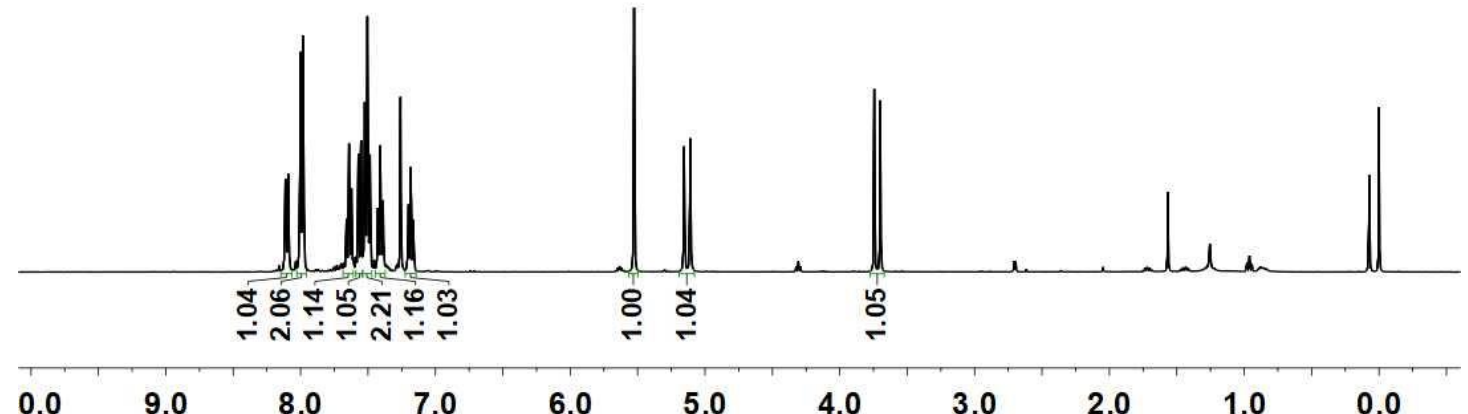

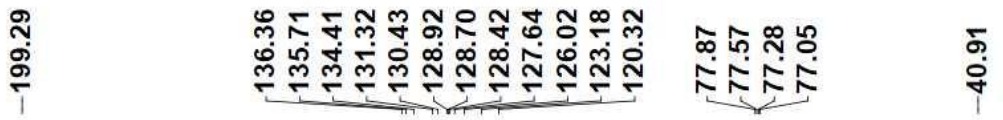

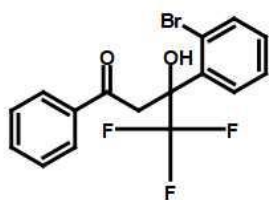

4b

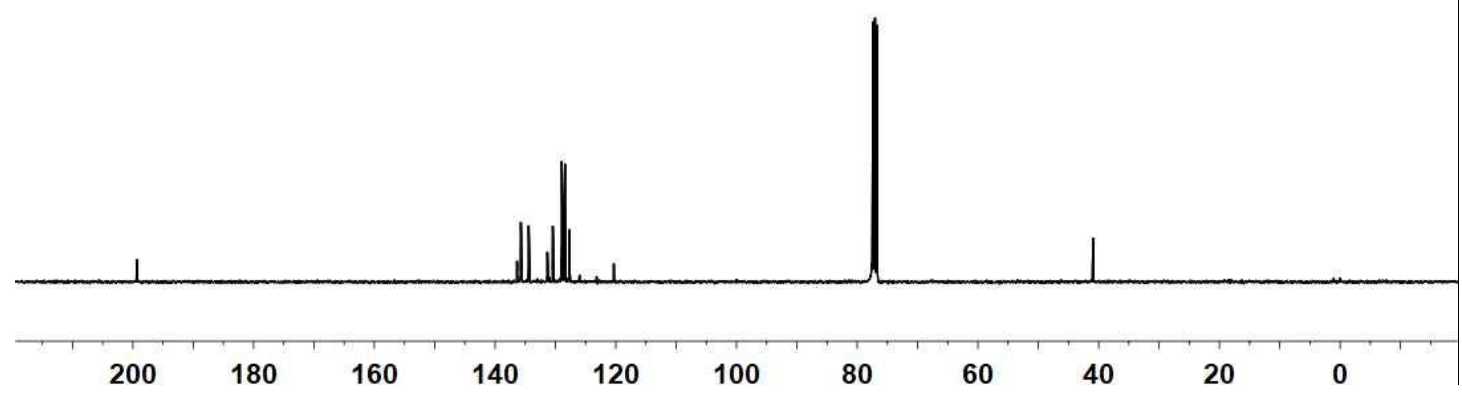




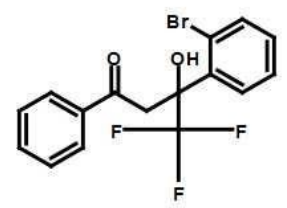

$4 b$

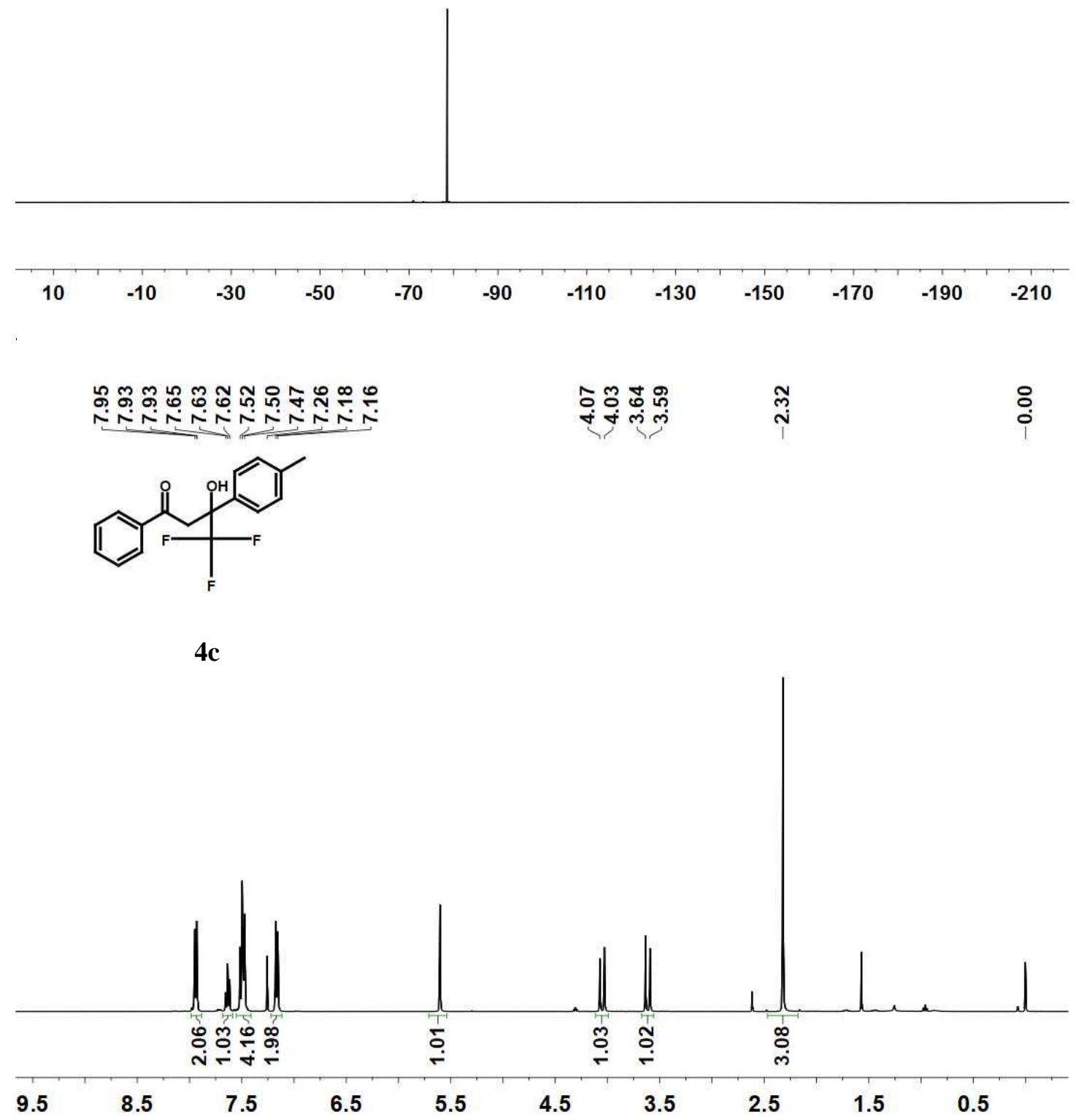


$\stackrel{\overline{0}}{\stackrel{1}{\circ}}$

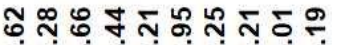

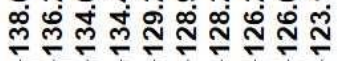

๕ ธุ กุ

象客

$\stackrel{\infty}{\stackrel{\circ}{\circ}}$<smiles>Cc1ccc(C(O)(CC(=O)c2ccccc2)C(F)(F)F)cc1</smiles>

$4 c$

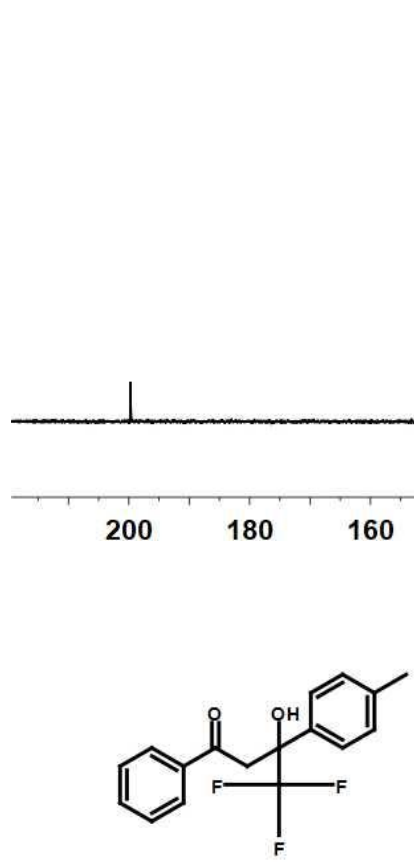

$4 c$

ষ্்

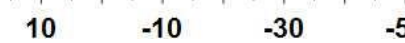

$-50 \quad-70$

$\begin{array}{lll}-90 & -110 & -130\end{array}$

$-150$

170

$-190 \quad-210$ 


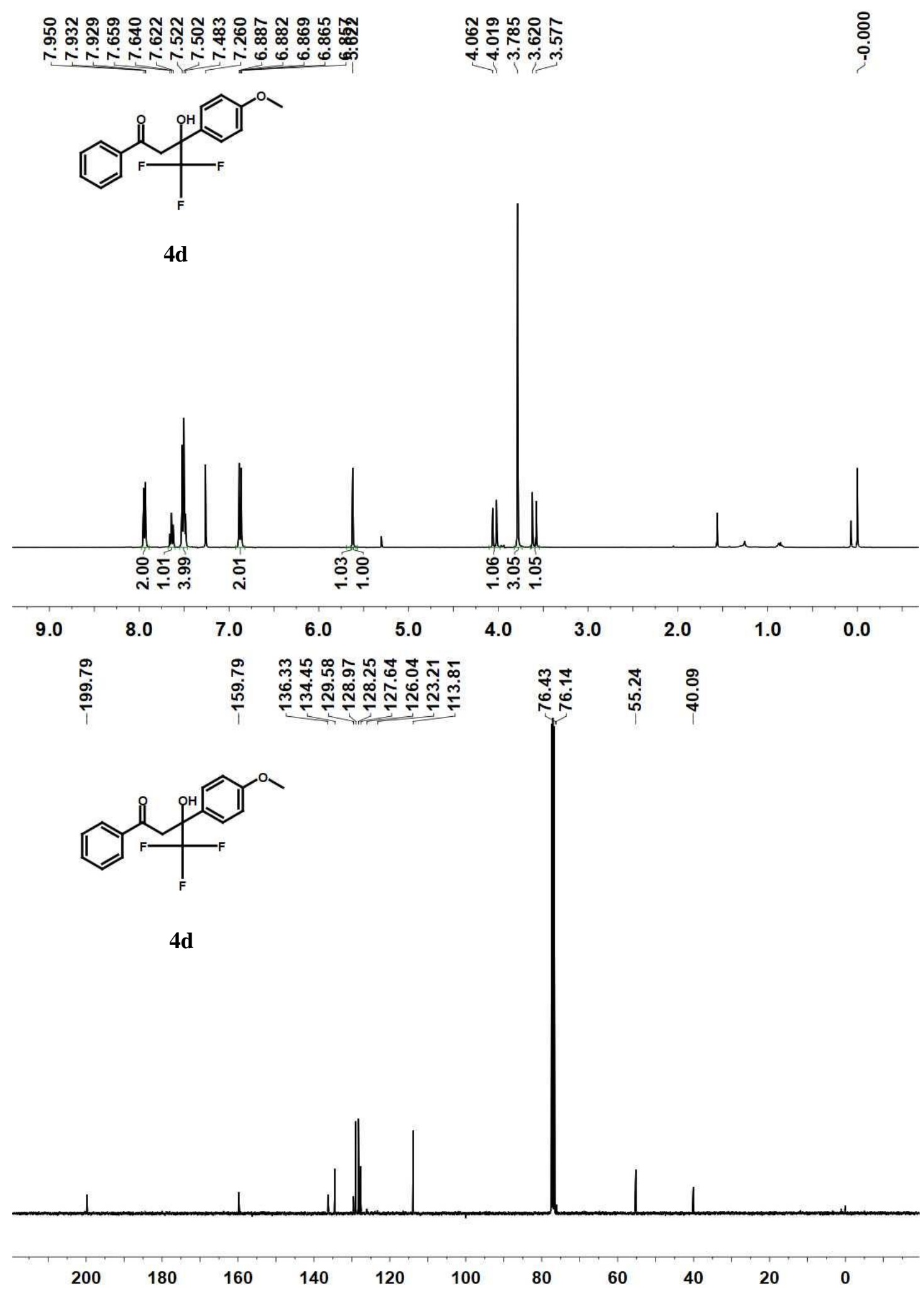




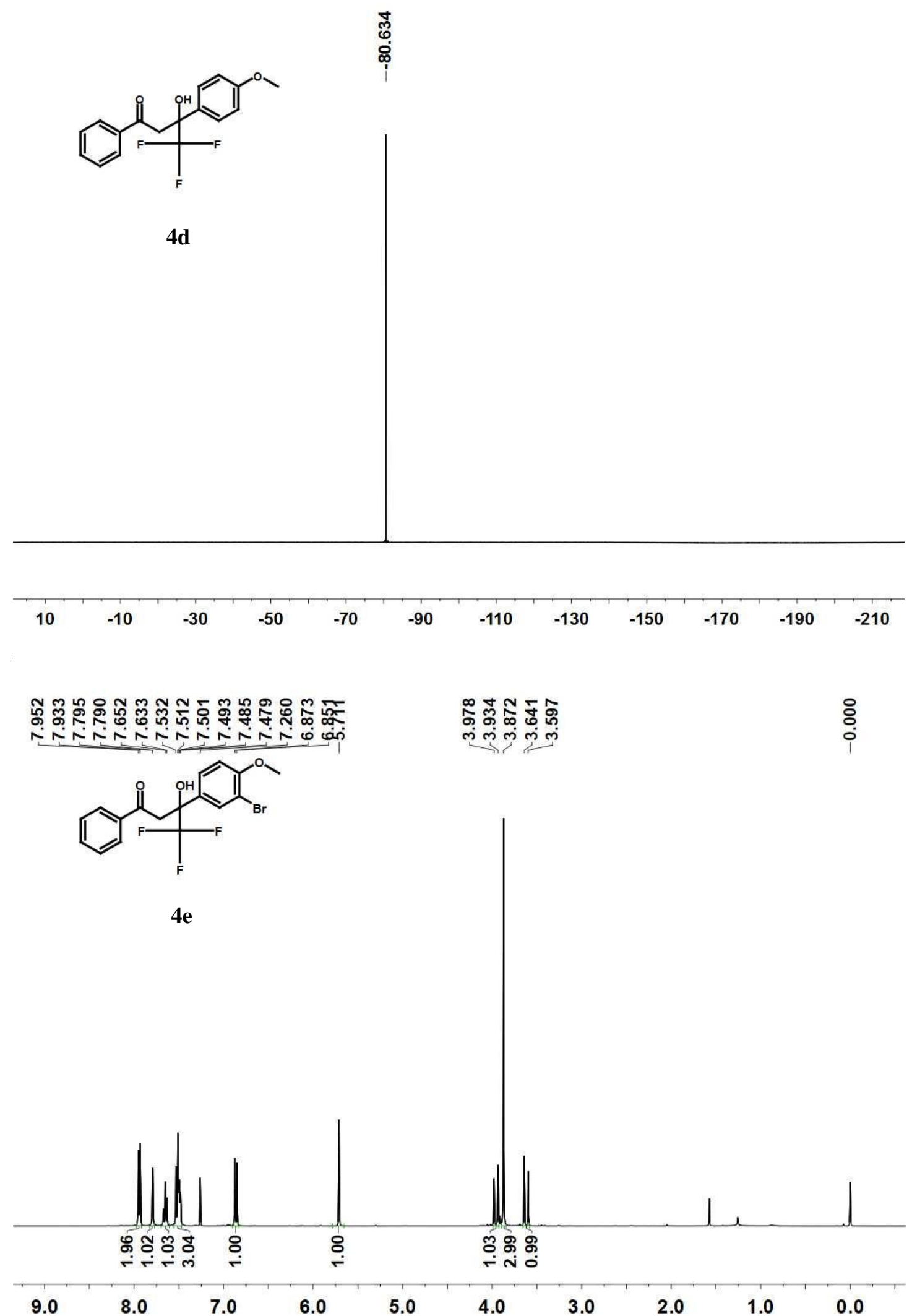




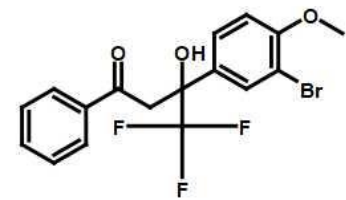

$4 e$

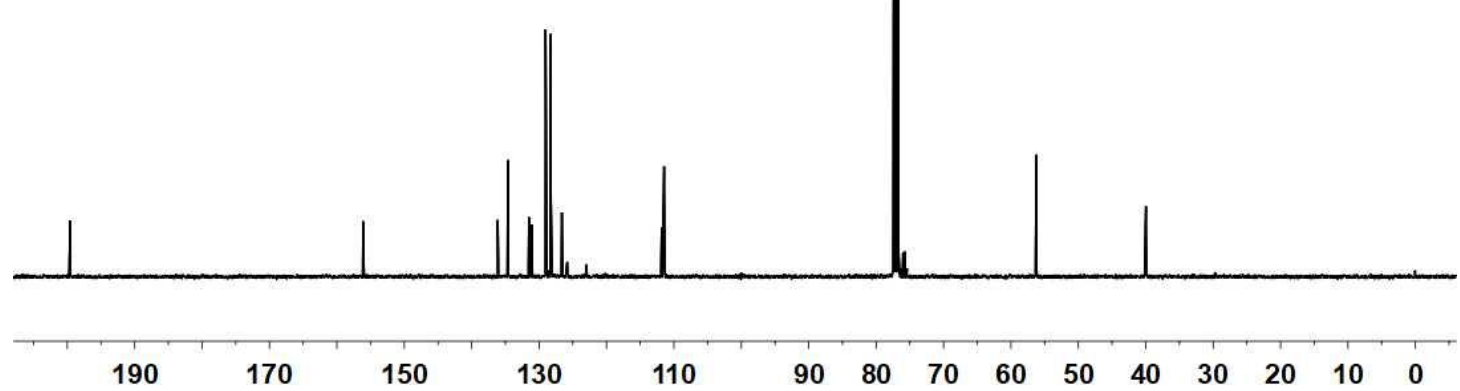

我<smiles>COc1ccc(C(O)(CC(=O)c2ccccc2)C(F)(F)F)cc1Br</smiles>

$4 e$

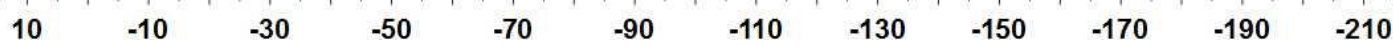




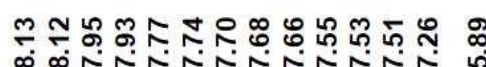

รูำร

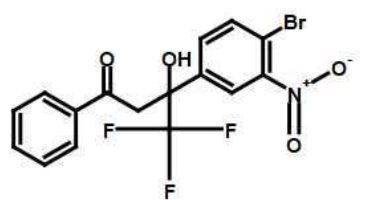

$4 \mathbf{f}$

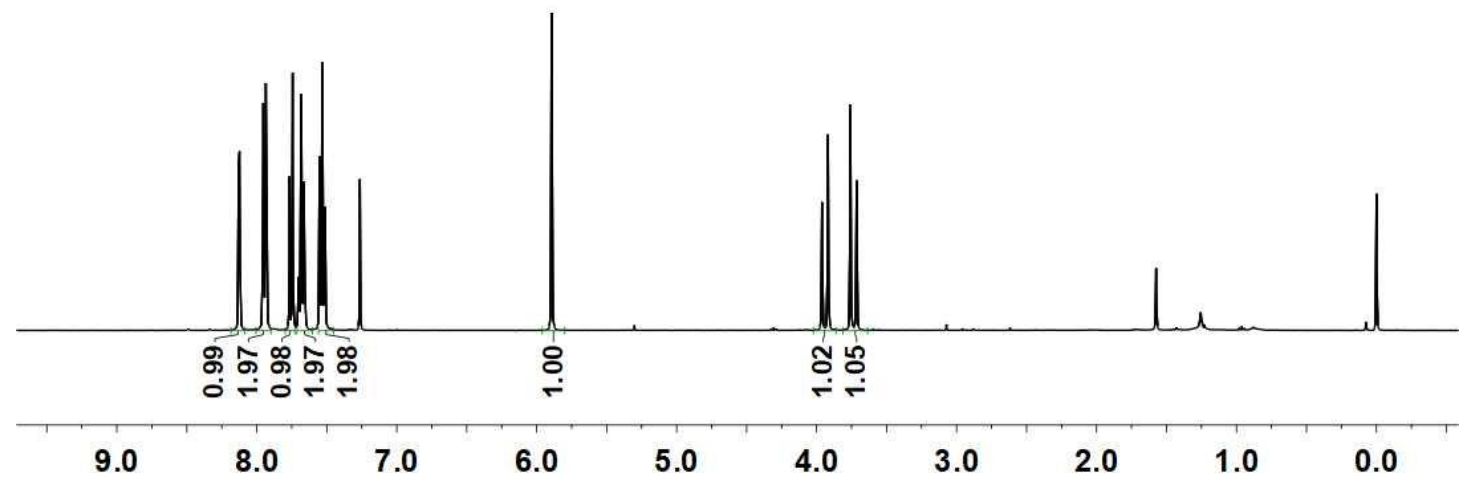

ㅇํㅎ

눈

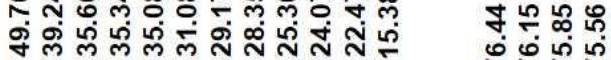

施它

స్ల

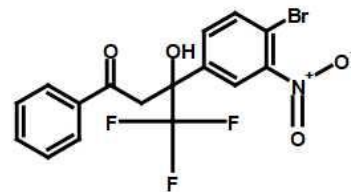

$4 f$

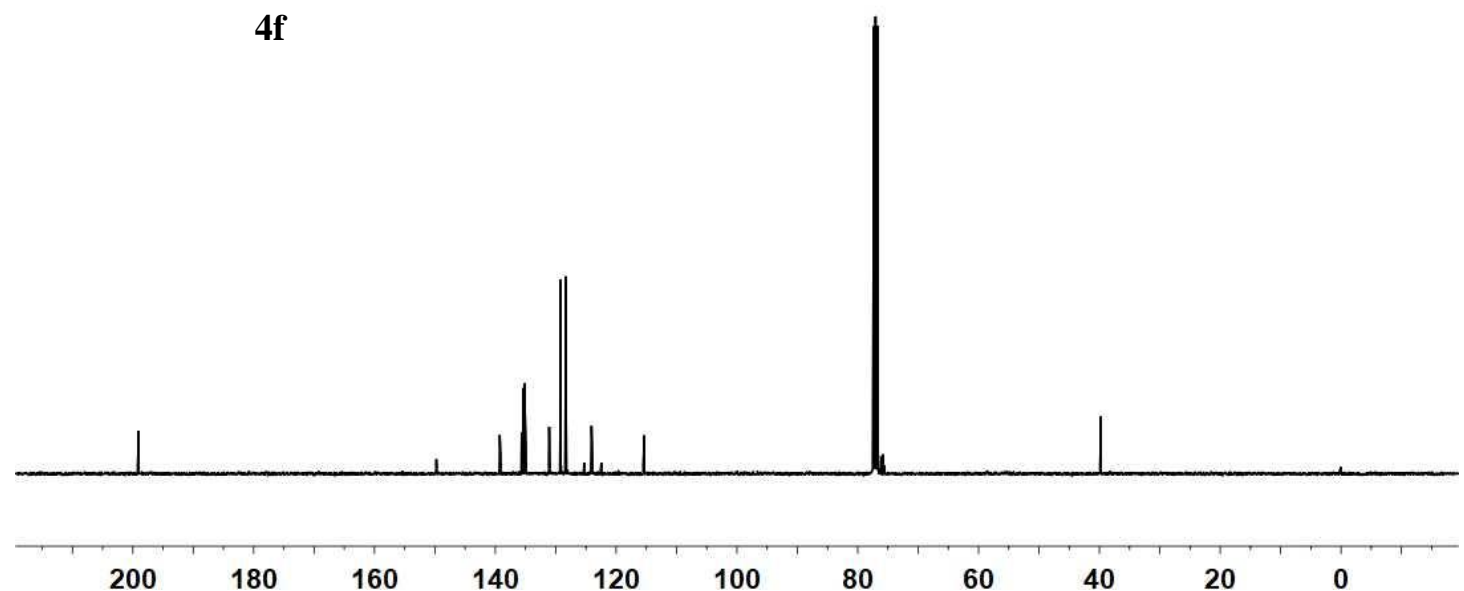




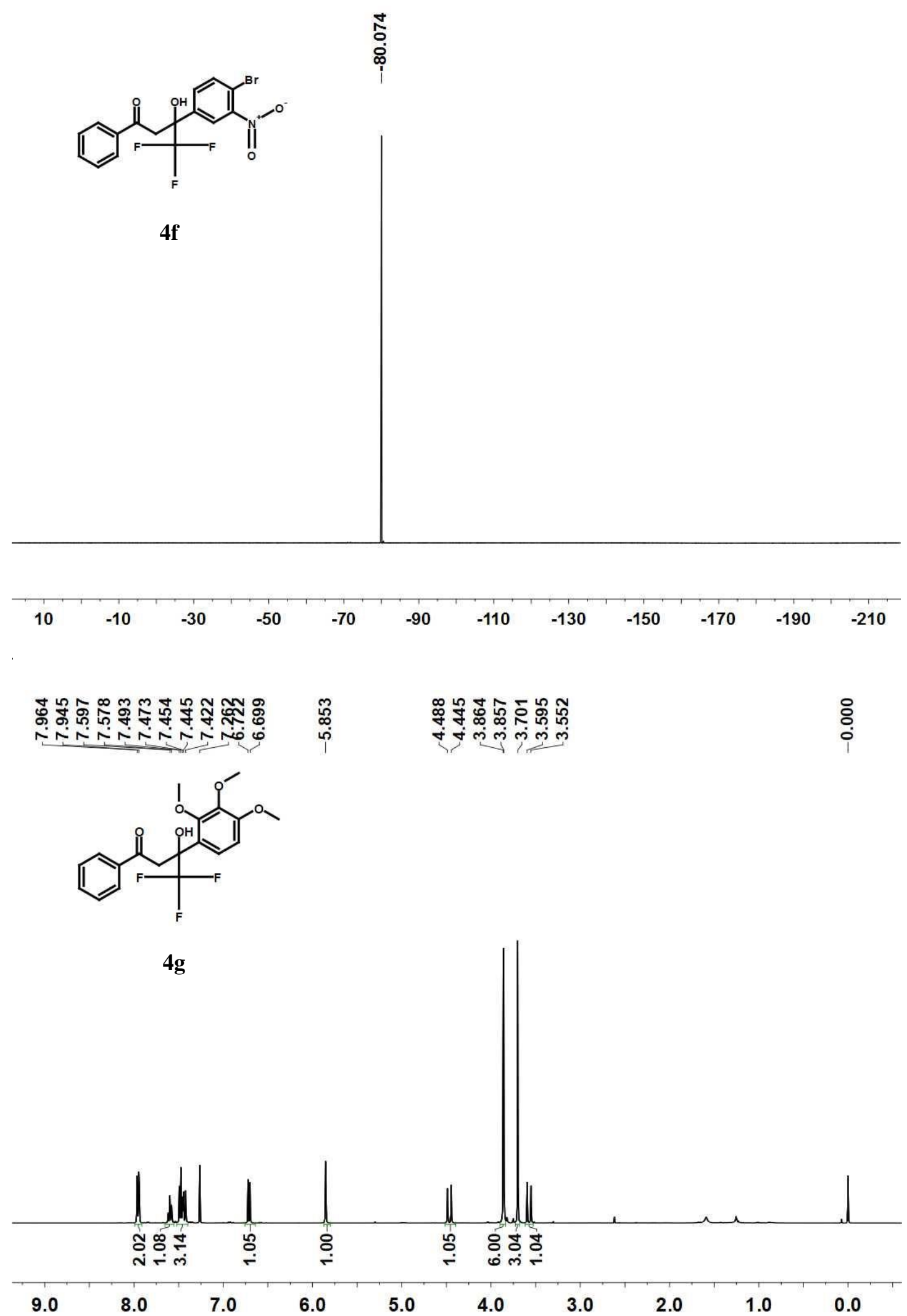


<smiles>COc1ccc(C(O)(CC(=O)c2ccccc2)C(F)(F)F)c(OC)c1OC</smiles>

$4 \mathrm{~g}$

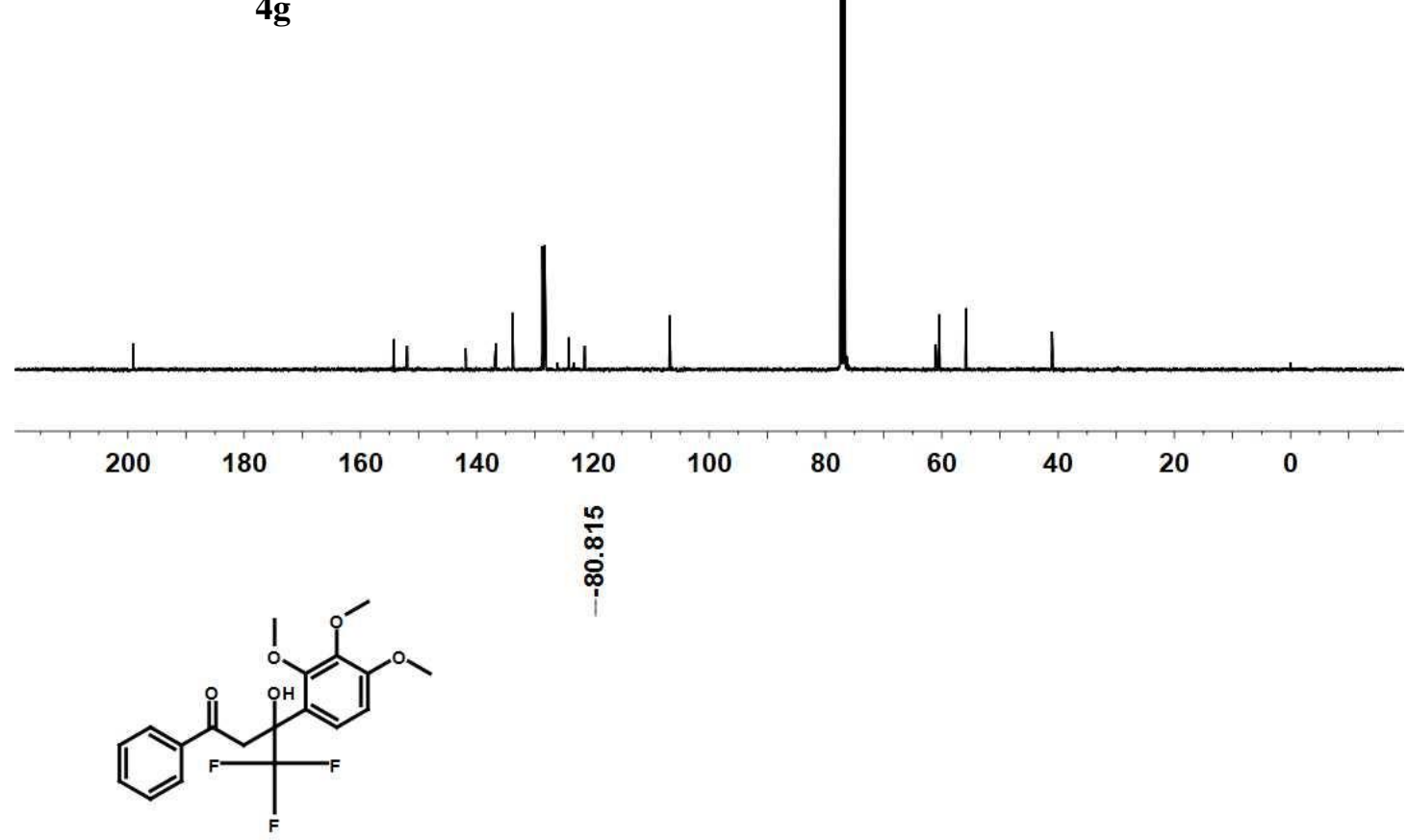

$4 \mathrm{~g}$

10

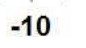

$-30 \quad-50$

$-70$

$-90$

$-110-130$

$-150$

$\begin{array}{lll}-170 & -190 & -210\end{array}$ 


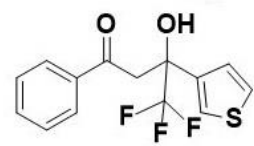

4h

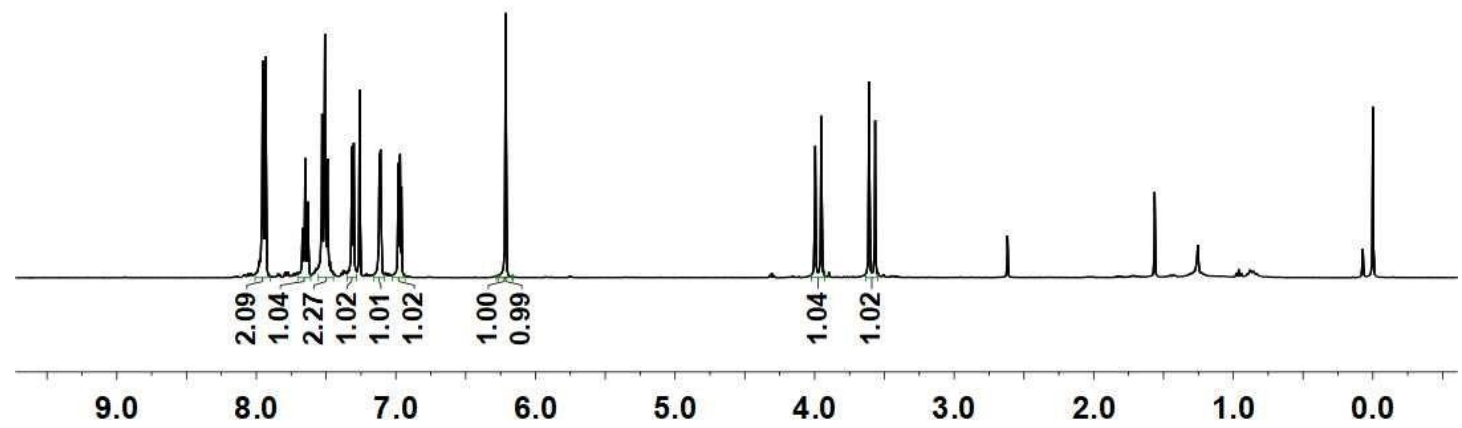

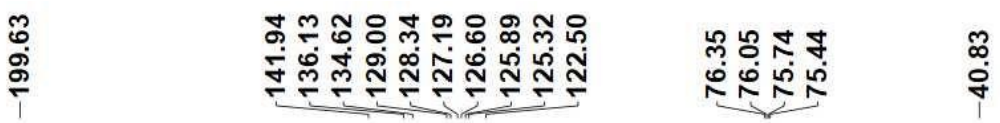

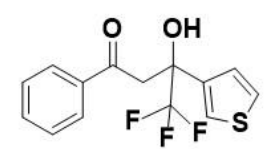

4h

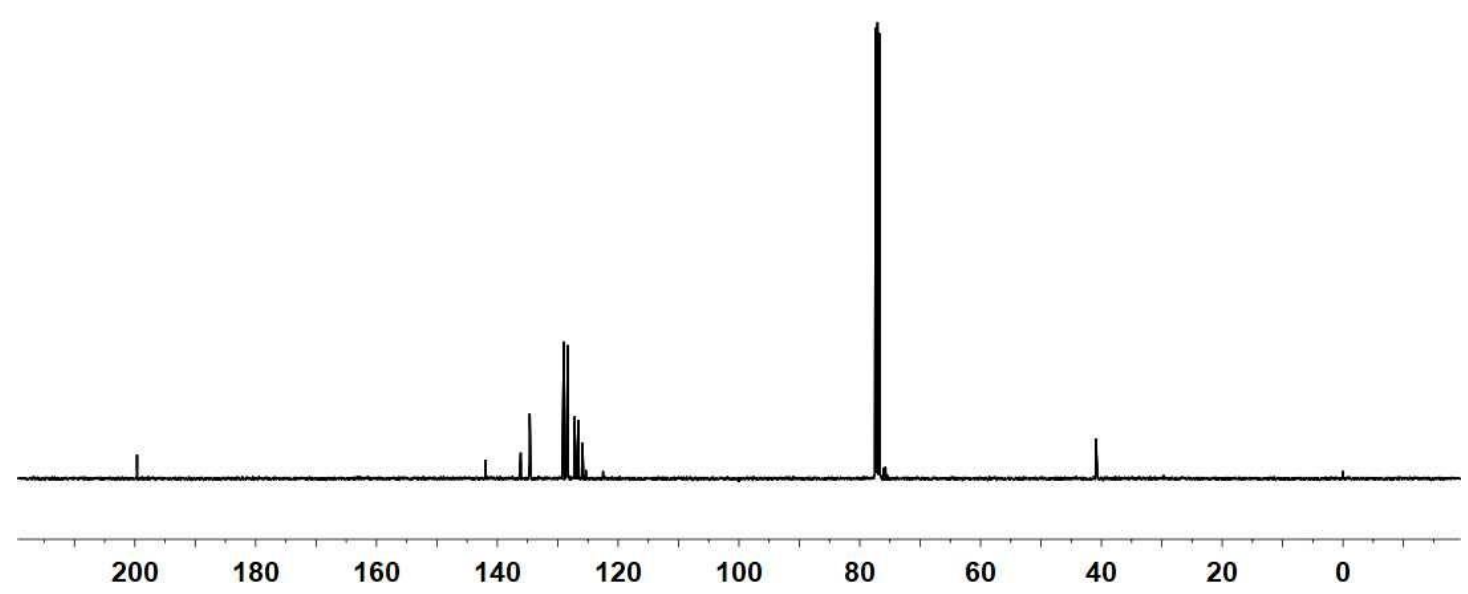




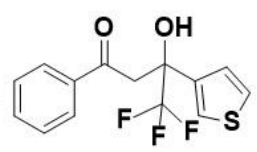

ஸ্ণ

4h

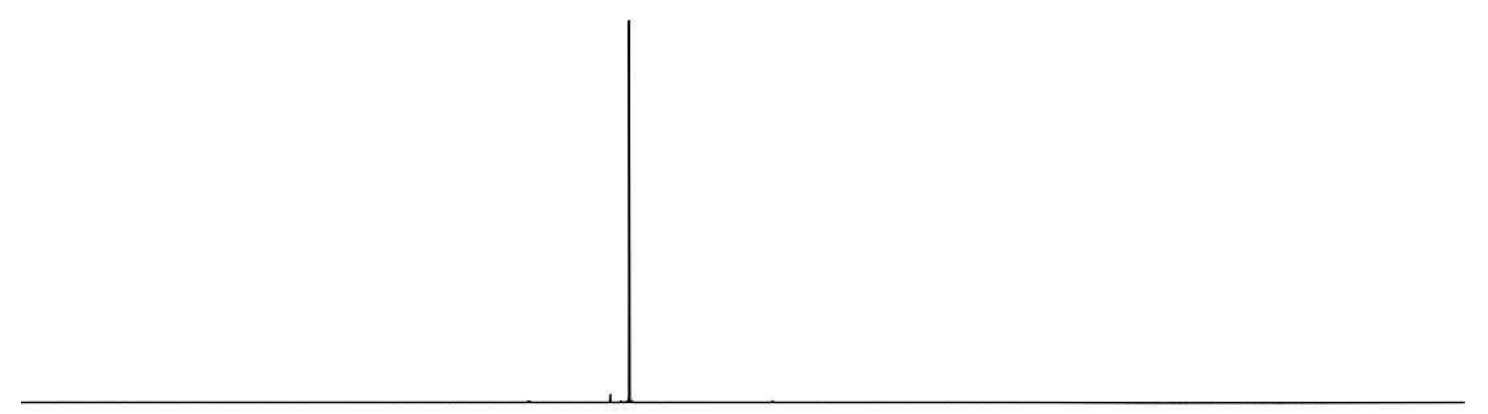

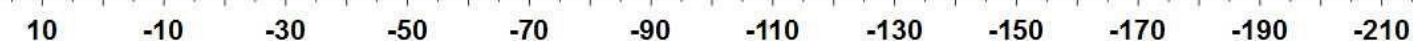

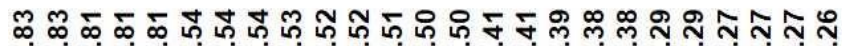

$\stackrel{8}{\substack{0 \\ i}}$

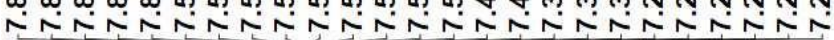
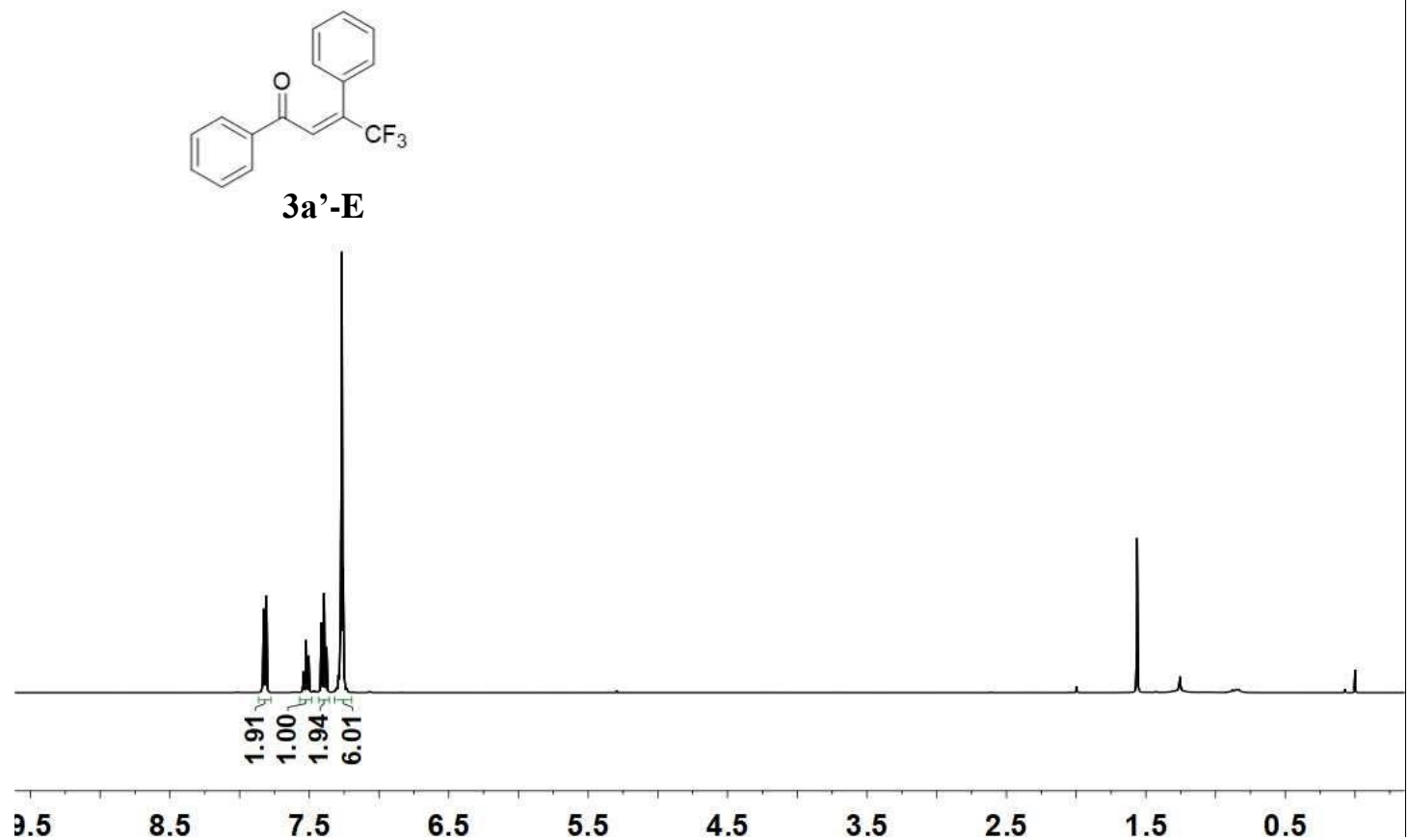


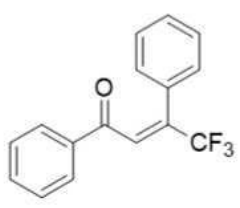

3a'-E
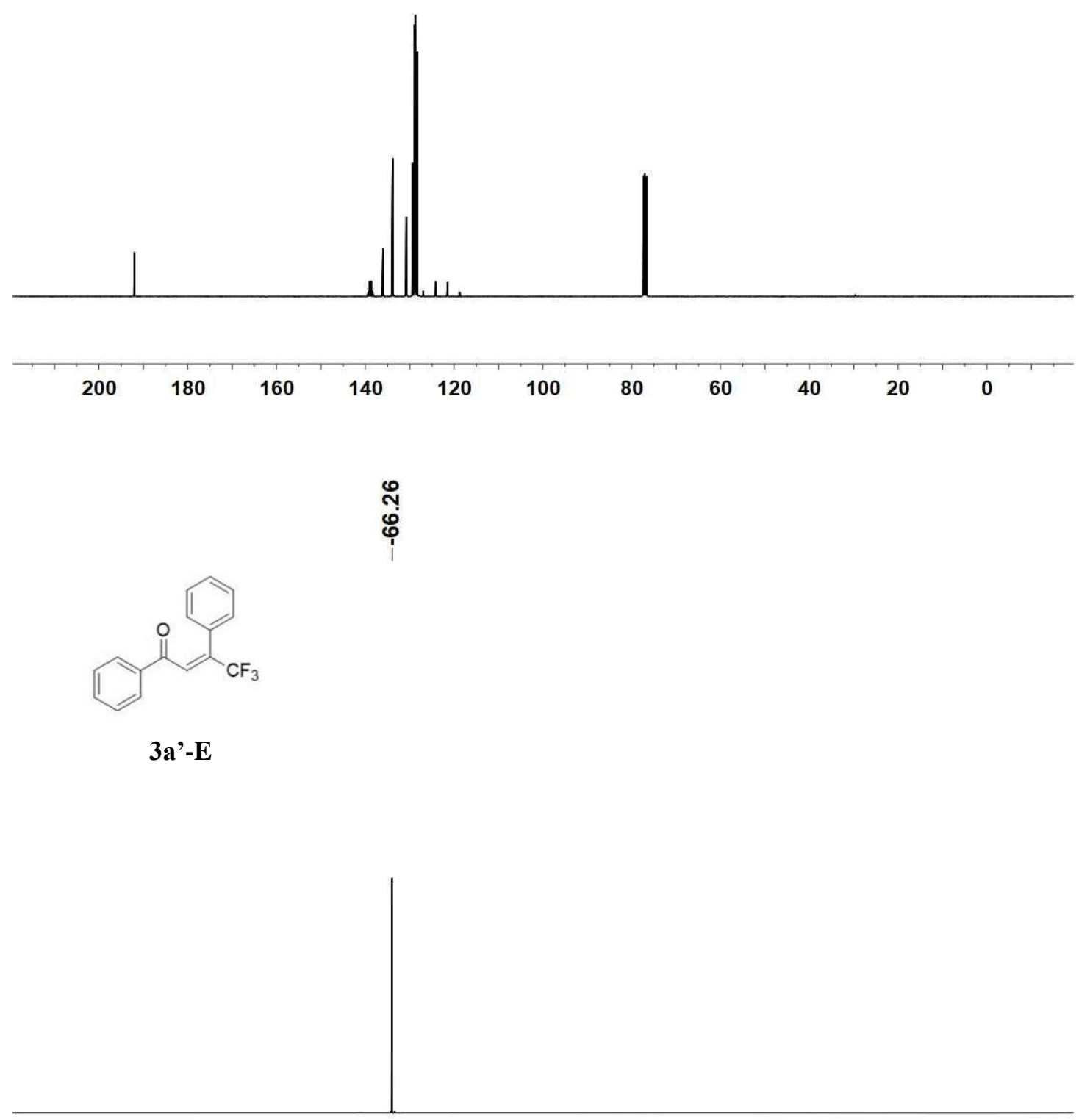

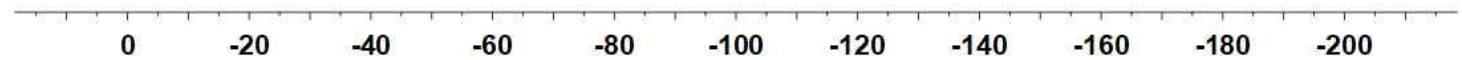



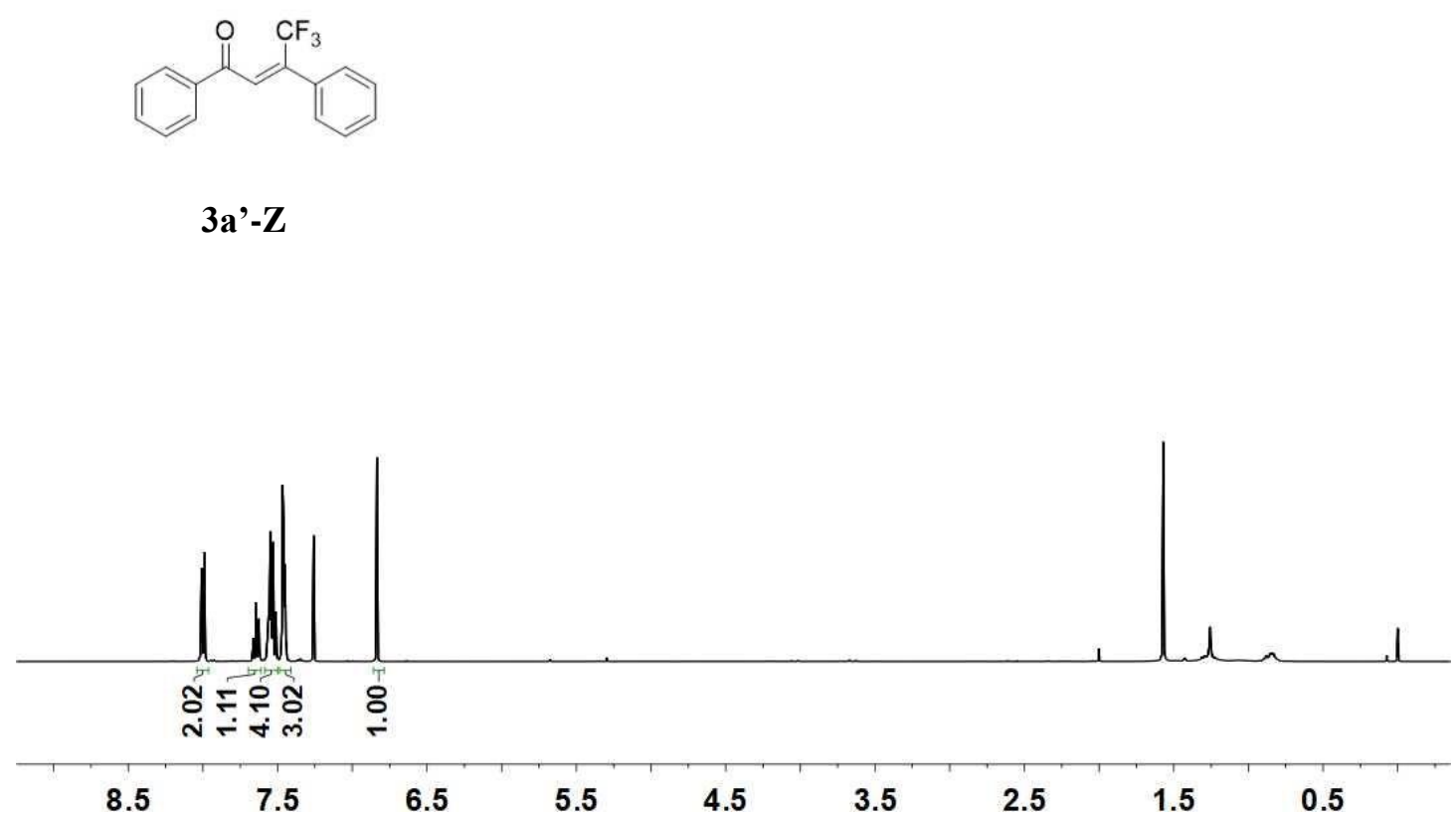

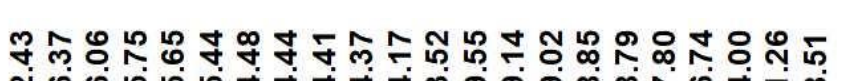

๙ู่

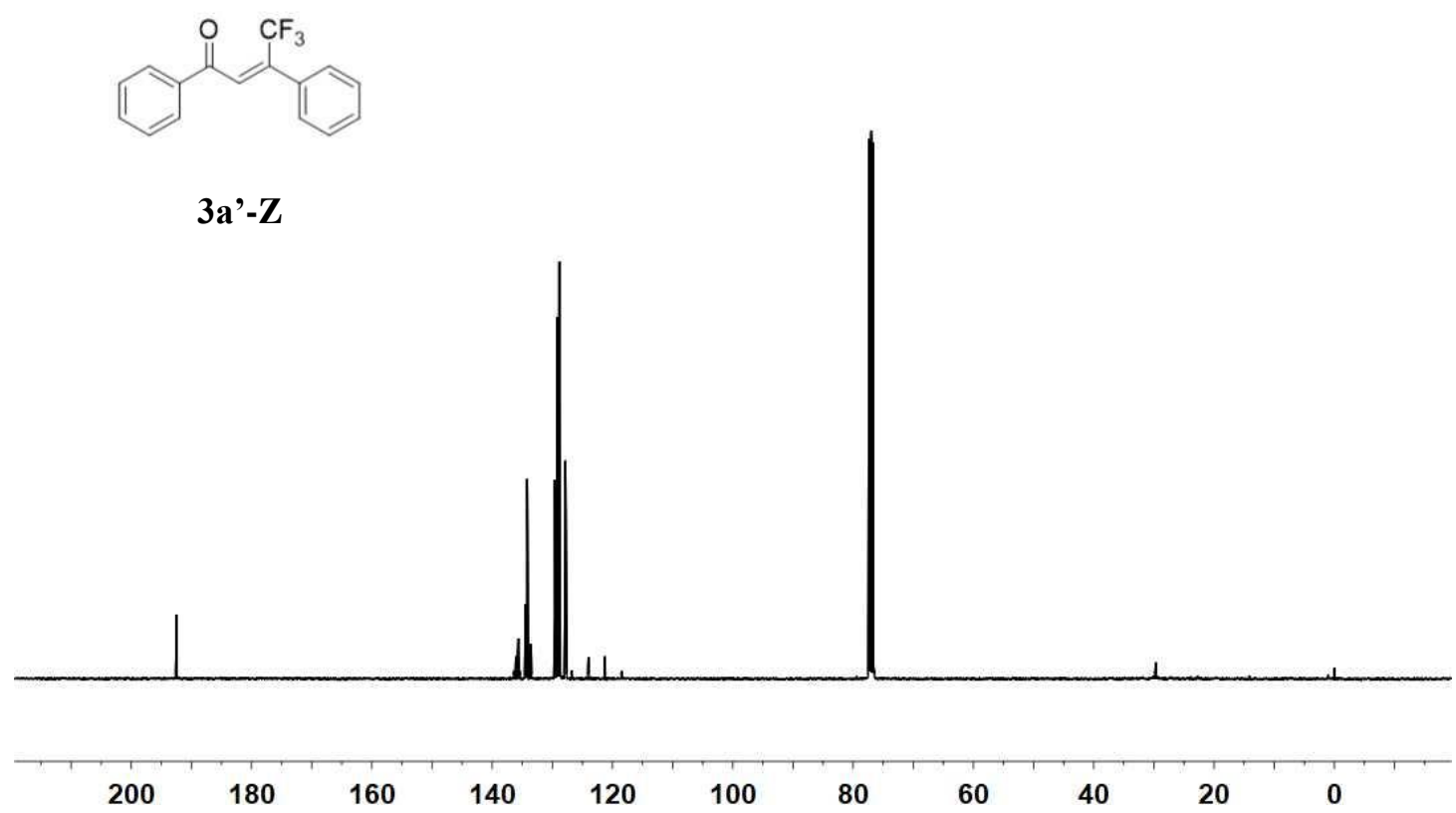


คั

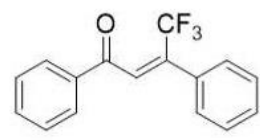

3a'-Z

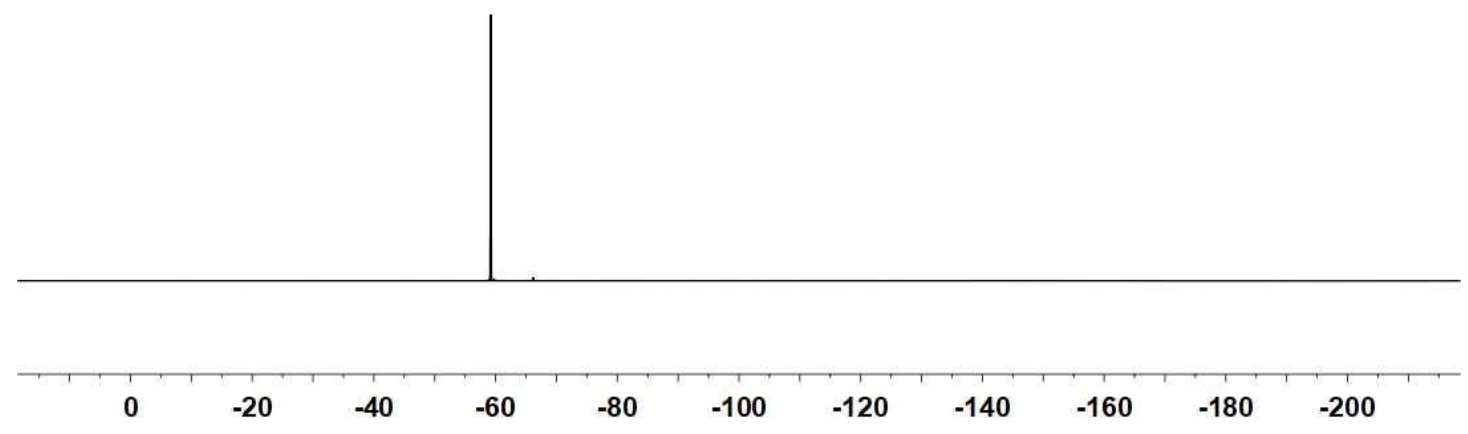

